Cochrane Database of Systematic Reviews

\title{
Shortened treatment regimens versus the standard regimen for drug-sensitive pulmonary tuberculosis (Review)
}

Grace AG, Mittal A, Jain S, Tripathy JP, Satyanarayana S, Tharyan P, Kirubakaran R

Grace AG, Mittal A, Jain S, Tripathy JP, Satyanarayana S, Tharyan P, Kirubakaran R.

Shortened treatment regimens versus the standard regimen for drug-sensitive pulmonary tuberculosis.

Cochrane Database of Systematic Reviews 2019, Issue 12. Art. No.: CD012918.

DOI: 10.1002/14651858.CD012918.pub2. 
TABLE OF CONTENTS

HEADER

ABSTRACT

PLAIN LANGUAGE SUMMARY

SUMMARY OF FINDINGS

BACKGROUND

OBJECTIVES

METHODS

RESULTS

Figure 1.

Figure 2.

Figure 3.

Figure 4.

Figure 5.

DISCUSSION

AUTHORS' CONCLUSIONS

ACKNOWLEDGEMENTS

REFERENCES

CHARACTERISTICS OF STUDIES

DATA AND ANALYSES

Analysis 1.1. Comparison 1 Moxifloxacin-containing 4-month ATT versus standard 6-month ATT regimens, Outcome 1 Relapse.

Analysis 1.2. Comparison 1 Moxifloxacin-containing 4-month ATT versus standard 6-month ATT regimens, Outcome 2 Relapse: subgroup analysis.

Analysis 1.3. Comparison 1 Moxifloxacin-containing 4-month ATT versus standard 6-month ATT regimens, Outcome 3 Relapse: sensitivity analysis accounting for missing data.

Analysis 1.4. Comparison 1 Moxifloxacin-containing 4-month ATT versus standard 6-month ATT regimens, Outcome 4 Death from any cause.

Analysis 1.5. Comparison 1 Moxifloxacin-containing 4-month ATT versus standard 6-month ATT regimens, Outcome 5 Treatment discontinuation.

Analysis 1.6. Comparison 1 Moxifloxacin-containing 4-month ATT versus standard 6-month ATT regimens, Outcome 6 Positive sputum culture/smear at 8 weeks.

Analysis 1.7. Comparison 1 Moxifloxacin-containing 4-month ATT versus standard 6-month ATT regimens, Outcome 7 Treatment failure.

Analysis 1.8. Comparison 1 Moxifloxacin-containing 4-month ATT versus standard 6-month ATT regimens, Outcome 8 Acquired drug resistance.

Analysis 1.9. Comparison 1 Moxifloxacin-containing 4-month ATT versus standard 6-month ATT regimens, Outcome 9 Serious adverse events.

Analysis 2.1. Comparison 2 Gatifloxacin-containing 4-month ATT versus standard 6-month ATT regimens, Outcome 1 Relapse.

Analysis 2.2. Comparison 2 Gatifloxacin-containing 4-month ATT versus standard 6-month ATT regimens, Outcome 2 Relapse: sensitivity analysis accounting for missing data.

Analysis 2.3. Comparison 2 Gatifloxacin-containing 4-month ATT versus standard 6-month ATT regimens, Outcome 3 Death from any cause.

Analysis 2.4. Comparison 2 Gatifloxacin-containing 4-month ATT versus standard 6-month ATT regimens, Outcome 4 Treatment discontinuation.

Analysis 2.5. Comparison 2 Gatifloxacin-containing 4-month ATT versus standard 6-month ATT regimens, Outcome 5 Positive sputum culture at 8 weeks.

Analysis 2.6. Comparison 2 Gatifloxacin-containing 4-month ATT versus standard 6-month ATT regimens, Outcome 6 Treatment failure.

Analysis 2.7. Comparison 2 Gatifloxacin-containing 4-month ATT versus standard 6-month ATT regimens, Outcome 7 Acquired drug resistance.

Analysis 2.8. Comparison 2 Gatifloxacin-containing 4-month ATT versus standard 6-month ATT regimens, Outcome 8 Serious adverse events.

ADDITIONAL TABLES

APPENDICES

Shortened treatment regimens versus the standard regimen for drug-sensitive pulmonary tuberculosis (Review) 
[Intervention Review]

\title{
Shortened treatment regimens versus the standard regimen for drug- sensitive pulmonary tuberculosis
}

\author{
Angeline G Grace1, Abhenil Mittal2 ${ }^{2}$, Siddharth Jain³, Jaya P Tripathy4, Srinath Satyanarayana5 ${ }^{5}$, Prathap Tharyan ${ }^{6}$, Richard Kirubakaran ${ }^{7}$ \\ 1Department of Community Medicine, Sree Balaji Medical College \& Hospital, Chennai, India. 2Department of Internal Medicine, All \\ India Institute of Medical Sciences, New Delhi, India. ${ }^{3}$ Clinical Immunology and Rheumatology Unit, Department of Internal Medicine, \\ Postgraduate Institute of Medical Education and Research (PGIMER), Chandigarh, India. ${ }^{4}$ Centre for Operational Research, International \\ Union Against Tuberculosis and Lung Disease (The Union), South-East Asia Regional Office, New Delhi, India. 5 International Union \\ Against Tuberculosis and Lung Disease (The Union), South-East Asia Regional Office, New Delhi, India. ${ }^{6}$ Clinical Epidemiology Unit, Prof. \\ BV Moses Centre for Evidence-Informed Healthcare and Health Policy, Christian Medical College, Vellore, India. ${ }^{7}$ Cochrane South Asia, \\ Prof. BV Moses Centre for Evidence-Informed Healthcare and Health Policy, Christian Medical College, Vellore, India
}

Contact address: Angeline G Grace, Department of Community Medicine, Sree Balaji Medical College \& Hospital, Works road, Chrompet, Chennai,600044, India. christoangeline@yahoo.com, angelinedr.g@gmail.com.

Editorial group: Cochrane Infectious Diseases Group.

Publication status and date: New, published in Issue 12, 2019.

Citation: Grace AG, Mittal A, Jain S, Tripathy JP, Satyanarayana S, Tharyan P, Kirubakaran R. Shortened treatment regimens versus the standard regimen for drug-sensitive pulmonary tuberculosis. Cochrane Database of Systematic Reviews 2019, Issue 12. Art. No.: CD012918. DOI: 10.1002/14651858.CD012918.pub2.

Copyright (c) 2019 The Authors. Cochrane Database of Systematic Reviews published by John Wiley \& Sons, Ltd. on behalf of The Cochrane Collaboration. This is an open access article under the terms of the Creative Commons Attribution-Non-Commercial Licence, which permits use, distribution and reproduction in any medium, provided the original work is properly cited and is not used for commercial purposes.

\section{A B S T R A C T}

\section{Background}

Tuberculosis causes more deaths than any other infectious disease worldwide, with pulmonary tuberculosis being the most common form. Standard first-line treatment for drug-sensitive pulmonary tuberculosis for six months comprises isoniazid, rifampicin, pyrazinamide, and ethambutol (HRZE) for two months, followed by HRE (in areas of high TB drug resistance) or HR, given over a four-month continuation phase. Many people do not complete this full course. Shortened treatment regimens that are equally effective and safe could improve treatment success.

\section{Objectives}

To evaluate the efficacy and safety of shortened treatment regimens versus the standard six-month treatment regimen for individuals with drug-sensitive pulmonary tuberculosis.

\section{Search methods}

We searched the following databases up to 10 July 2019: the Cochrane Infectious Diseases Group Specialized Register; the Central Register of Controlled Trials (CENTRAL), in the Cochrane Library; MEDLINE (PubMed); Embase; the Latin American Caribbean Health Sciences Literature (LILACS); Science Citation Index-Expanded; Indian Medlars Center; and the South Asian Database of Controlled Clinical Trials. We also searched the World Health Organization (WHO) International Clinical Trials Registry Platform, ClinicalTrials.gov, the Clinical Trials Unit of the International Union Against Tuberculosis and Lung Disease, the UK Medical Research Council Clinical Trials Unit, and the Clinical Trials Registry India for ongoing trials. We checked the reference lists of identified articles to find additional relevant studies. 


\section{Selection criteria}

We searched for randomized controlled trials (RCTs) or quasi-RCTs that compared shorter-duration regimens (less than six months) versus the standard six-month regimen for people of all ages, irrespective of HIV status, who were newly diagnosed with pulmonary tuberculosis by positive sputum culture or GeneXpert, and with presumed or proven drug-sensitive tuberculosis. The primary outcome of interest was relapse within two years of completion of anti-tuberculosis treatment (ATT).

\section{Data collection and analysis}

Two review authors independently selected trials, extracted data, and assessed risk of bias for the included trials. For dichotomous outcomes, we used risk ratios (RRs) with 95\% confidence intervals (Cls). When appropriate, we pooled data from the included trials in meta-analyses. We assessed the certainty of evidence using the GRADE approach.

\section{Main results}

We included five randomized trials that compared fluoroquinolone-containing four-month ATT regimens versus standard six-month ATT regimens and recruited 5825 adults with newly diagnosed drug-sensitive pulmonary tuberculosis from 14 countries with high tuberculosis transmission in Asia, Africa, and Latin Ameria. Three were multi-country trials that included a total of 572 HIV-positive people. These trials excluded children, pregnant or lactating women, people with serious comorbid conditions, and those with diabetes mellitus. Four trials had multiple treatment arms.

Moxifloxacin replaced ethambutol in standard four-month, daily or thrice-weekly ATT regimens in two trials; moxifloxacin replaced isoniazid in four-month ATT regimens in two trials, was given daily in one trial, and was given with rifapentine instead of rifampicin daily for two months and twice weekly for two months in one trial. Moxifloxacin was added to standard ATT drugs for three to four months in one ongoing trial that reported interim results. Gatifloxacin replaced ethambutol in standard ATT regimens given daily or thrice weekly for four months in two trials. Follow-up ranged from 12 months to 24 months after treatment completion for the majority of participants.

\section{Moxifloxacin-containing four-month ATT regimens}

Moxifloxacin-containing four-month ATT regimens that replaced ethambutol or isoniazid probably increased the proportions who experienced relapse after successful treatment compared to standard ATT regimens (RR 3.56, 95\% Cl 2.37 to $5.37 ; 2265$ participants, 3 trials; moderate-certainty evidence). For death from any cause, there was probably little or no difference between the two regimens ( 2760 participants, 3 trials; moderate-certainty evidence). Treatment failure was rare, and there was probably little or no difference in proportions with treatment failure between ATT regimens (2282 participants, 3 trials; moderate-certainty evidence). None of the participants given moxifloxacin-containing regimens developed resistance to rifampicin, and these regimens may not increase the risk of acquired resistance (2282 participants, 3 trials; low-certainty evidence). Severe adverse events were probably little or no different with moxifloxacin-containing four-month regimens that replaced ethambutol or isoniazid, and with three- to four-month regimens that augmented standard ATT with moxifloxacin, when compared to standard six-month ATT regimens (3548 participants, 4 trials; moderate-certainty evidence).

\section{Gatifloxacin-containing four-month ATT regimens}

Gatifloxacin-containing four-month ATT regimens that replaced ethambutol probably increased relapse compared to standard six-month ATT regimens in adults with drug-sensitive pulmonary tuberculosis (RR 2.11, 95\% Cl 1.56 to 2.84; 1633 participants, 2 trials; moderatecertainty evidence). The four-month regimen probably made little or no difference in death compared to the six-month regimen (1886 participants, 2 trials; moderate-certainty evidence). Treatment failure was uncommon and was probably little or no different between the four-month and six-month regimens (1657 participants, 2 trials; moderate-certainty evidence). Acquired resistance to isoniazid or rifampicin was not detected in those given the gatifloxacin-containing shortened ATT regimen, but we are uncertain whether acquired drug resistance is any different in the four- and six-month regimens (429 participants, 1 trial; very low-certainty evidence). Serious adverse events were probably no different with either regimen (1993 participants, 2 trials; moderate-certainty evidence).

\section{Authors' conclusions}

Evidence to date does not support the use of shortened ATT regimens in adults with newly diagnosed drug-sensitive pulmonary tuberculosis. Four-month ATT regimens that replace ethambutol with moxifloxacin or gatifloxacin, or isoniazid with moxifloxacin, increase relapse substantially compared to standard six-month ATT regimens, although treatment success and serious adverse events are little or no different. The results of six large ongoing trials will help inform decisions on whether shortened ATT regimens can replace standard sixmonth ATT regimens.

\section{December 2019}

Up to date

All studies incorporated from most recent search

All eligible published studies found in the last search (10 Jul, 2019) were included 


\section{PLAIN LANGUAGE SUMMARY}

\section{Shorter treatment regimens for people with pulmonary tuberculosis}

\section{What was the aim of this review?}

The aim of this Cochrane Review was to find out if the duration of anti-tuberculosis treatment (ATT) for people with newly diagnosed drugsensitive pulmonary tuberculosis can be shortened to less than six months. Cochrane Review authors collected and analysed all relevant studies to answer this question and found five relevant studies.

\section{Key messages}

Shortened ATT regimens probably make little or no difference in death, treatment failure, or serious adverse events compared to six-month ATT regimens, but they probably increase relapse of tuberculosis. Six large ongoing trials are studying this question.

\section{What was studied in the review?}

Tuberculosis is an infectious disease, and tuberculosis affecting the lungs (pulmonary tuberculosis) is the most common presentation of tuberculosis in adults. Tuberculosis is a major public health problem worldwide, and among infectious diseases, it is the leading cause of death.

People with pulmonary tuberculosis are currently treated with a six-month combination of drugs that include isoniazid, rifampicin, ethambutol, and pyrazinamide for two months, followed by isoniazid and rifampicin (with or without ethambutol) for four months. Many people do not finish the treatment or they take the drugs irregularly because of the long treatment duration, or because of drug side effects. Incomplete or irregular treatment can lead to treatment failure and can increase disease relapse. Such treatment can also lead to drug resistance. If newer drug combinations given for less than six months are found to be as effective and safe as the currently recommended six-month ATT regimens, more people might be adherent and might complete treatment. This could help reduce drug resistance and could help to stop tuberculosis infection worldwide.

\section{What are the main results of the review?}

The five included trials studied 5825 adults with newly diagnosed drug-sensitive pulmonary tuberculosis from 14 countries with high tuberculosis transmission in Asia, Africa, and Latin Ameria. Three trials included 572 HIV-positive people, but all excluded people with other serious comorbid conditions and those with diabetes mellitus. This reduced the applicability of study results. All were funded by government or international agencies.

Four studies replaced isoniazid or ethambutol with moxifloxacin or gatifloxacin in four-month ATT regimens. Follow-up was provided for 12 months to 24 months after treatment completion. In one ongoing study, moxifloxacin was added to four-month ATT, but study authors provided only interim results.

This review shows the following when four-month ATT regimens are compared to standard six-month ATT regimens.

- Relapse after successful treatment is probably increased (moderate-certainty evidence).

- Death from any cause, treatment failure, and serious adverse events are probably little or no different (moderate-certainty evidence). - Drug resistance may not be increased with moxifloxacin-containing four-month regimens (low-certainty evidence), but we are uncertain whether this applies to gatifloxacin-containing regimens (very low-certainty evidence).

\section{How up-to-date is this review?}

The review authors searched for available studies up to 10 July 2019. 


\section{S U M MARY OF F I N D I G S}

Summary of findings for the main comparison. Moxifloxacin-containing 4-month ATT regimens versus standard 6-month ATT regimen for drugsensitive pulmonary tuberculosis

\section{Moxifloxacin-containing 4-month ATT versus standard 6-month ATT regimen for drug-sensitive pulmonary tuberculosis}

Patient or population: adults with drug-sensitive pulmonary tuberculosis

Setting: low- and middle-income countries in Africa, Asia, and Latin America

Intervention: moxifloxacin-containing 4-month ATT

Comparison: standard 6-month ATT

\begin{tabular}{|c|c|c|c|c|c|c|}
\hline \multirow[t]{2}{*}{ Outcomes } & \multicolumn{2}{|c|}{ Anticipated absolute effects ${ }^{\star}(95 \% \mathrm{Cl})$} & \multirow{2}{*}{$\begin{array}{l}\text { Relative effect } \\
(95 \% \mathrm{Cl})\end{array}$} & \multirow{2}{*}{$\begin{array}{l}\text { Number of par- } \\
\text { ticipants (stud- } \\
\text { ies) }\end{array}$} & \multirow{2}{*}{$\begin{array}{l}\text { Certainty of } \\
\text { the evidence } \\
\text { (GRADE) }\end{array}$} & \multirow[t]{2}{*}{ Comments } \\
\hline & $\begin{array}{l}\text { Risk with 6- } \\
\text { month stan- } \\
\text { dard ATT }\end{array}$ & $\begin{array}{l}\text { Risk with 4-month moxi- } \\
\text { floxacin-containing ATT }\end{array}$ & & & & \\
\hline $\begin{array}{l}\text { Relapse } \\
\text { Follow-up: } \\
\text { range } 12 \text { to } 24 \\
\text { months }\end{array}$ & 32 per 1000 & $\begin{array}{l}82 \text { more relapses per } 1000 \\
\text { (44 more to } 140 \text { more) }\end{array}$ & $\begin{array}{l}\text { RR } 3.56 \\
\text { (2.37 to } 5.37)\end{array}$ & $\begin{array}{l}2265 \\
\text { (3 RCTs) }\end{array}$ & $\begin{array}{l}\oplus \oplus \oplus \ominus \\
\text { MODERATEa,b,c } \\
\begin{array}{l}\text { Due to indirect- } \\
\text { ness }\end{array}\end{array}$ & $\begin{array}{l}\text { The 4-month regimen probably increases } \\
\text { relapse compared to the 6-month regimen }\end{array}$ \\
\hline $\begin{array}{l}\text { Death from } \\
\text { any cause } \\
\text { Follow-up: } \\
\text { range } 18 \text { to } 24 \\
\text { months }\end{array}$ & 21 per 1000 & $\begin{array}{l}2 \text { more deaths per } 1000 \\
\text { ( } 7 \text { fewer to } 16 \text { more) }\end{array}$ & $\begin{array}{l}\text { RR } 1.06 \\
\text { (0.65 to } 1.75)\end{array}$ & $\begin{array}{l}2760 \\
\text { (3 RCTs) }\end{array}$ & $\begin{array}{l}\oplus \oplus \oplus \ominus \\
\text { MODERATEa,c,d } \\
\text { Due to indirect- } \\
\text { ness }\end{array}$ & $\begin{array}{l}\text { The 4-month regimen probably makes lit- } \\
\text { tle or no difference in death from any cause } \\
\text { compared to the } 6 \text {-month regimen }\end{array}$ \\
\hline $\begin{array}{l}\text { Treatment fail- } \\
\text { ure }\end{array}$ & 16 per 1000 & $\begin{array}{l}5 \text { fewer treatment failures } \\
\text { per } 1000 \\
\text { (11 fewer to } 8 \text { more) }\end{array}$ & $\begin{array}{l}\text { RR } 0.71 \\
\text { (0.33 to } 1.52)\end{array}$ & $\begin{array}{l}2282 \\
\text { (3 RCTs) }\end{array}$ & $\begin{array}{l}\oplus \oplus \oplus \ominus \\
\text { MODERATEa,c,d } \\
\text { Due to indirect- } \\
\text { ness }\end{array}$ & $\begin{array}{l}\text { The 4-month regimen probably results in } \\
\text { little or no difference in treatment failure } \\
\text { compared to the } 6 \text {-month regimen }\end{array}$ \\
\hline $\begin{array}{l}\text { Acquired drug } \\
\text { resistance }\end{array}$ & 7 per 1000 & $\begin{array}{l}5 \text { fewer with acquired } \\
\text { drug resistance per } 1000 \\
\text { ( } 6 \text { fewer to } 2 \text { more) }\end{array}$ & $\begin{array}{l}\text { RR } 0.33 \\
\text { (0.08 to } 1.31)\end{array}$ & $\begin{array}{l}2282 \\
(3 \mathrm{RCTs}) \mathrm{e}\end{array}$ & $\begin{array}{l}\oplus \oplus \ominus \ominus \\
\text { LOWc,f,g } \\
\text { Due to indirect- } \\
\text { ness and impreci- } \\
\text { sion }\end{array}$ & $\begin{array}{l}\text { The 4-month regimen may be little or no } \\
\text { different than the } 6 \text {-month regimen in the } \\
\text { incidence of acquired drug resistance }\end{array}$ \\
\hline
\end{tabular}




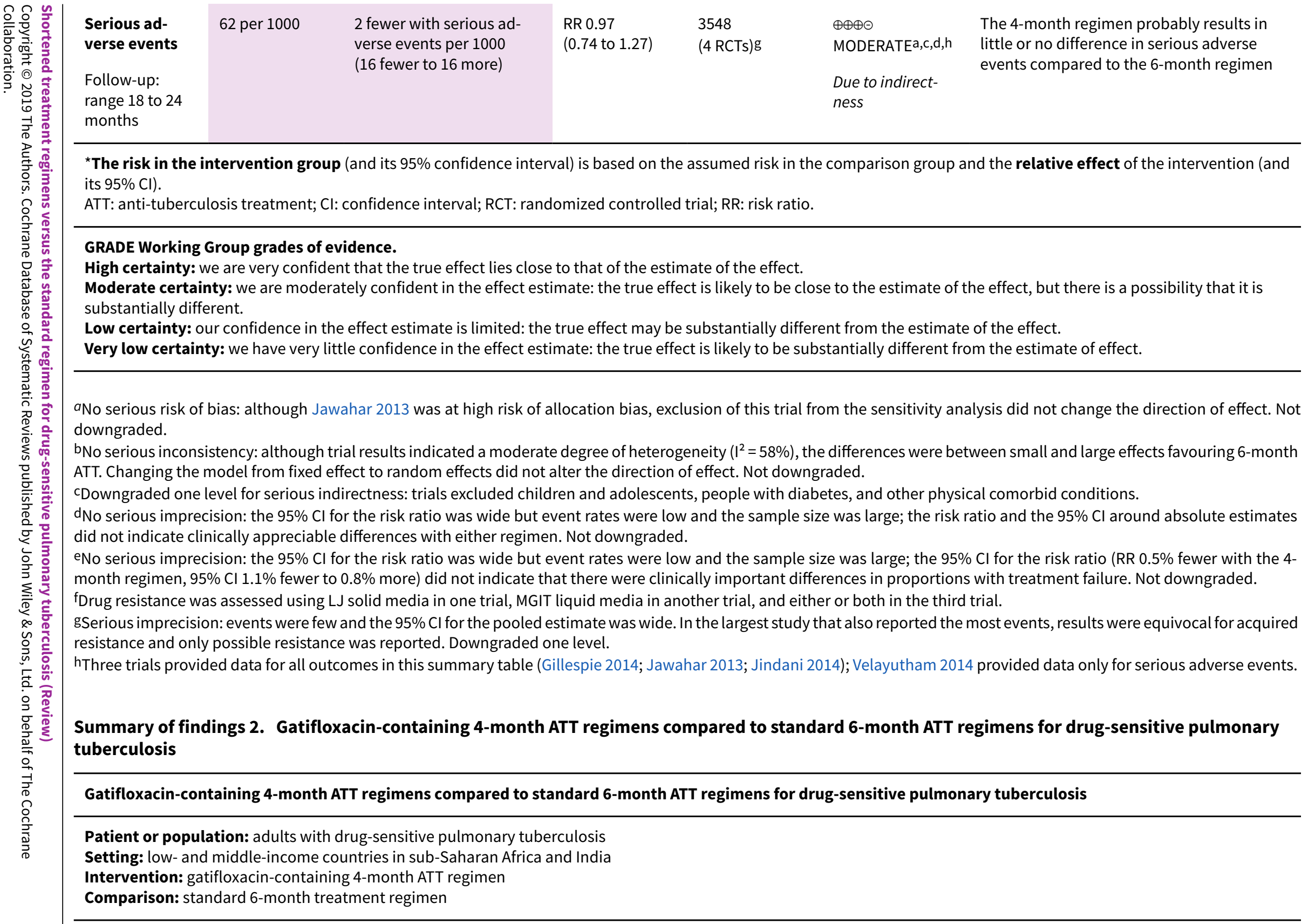




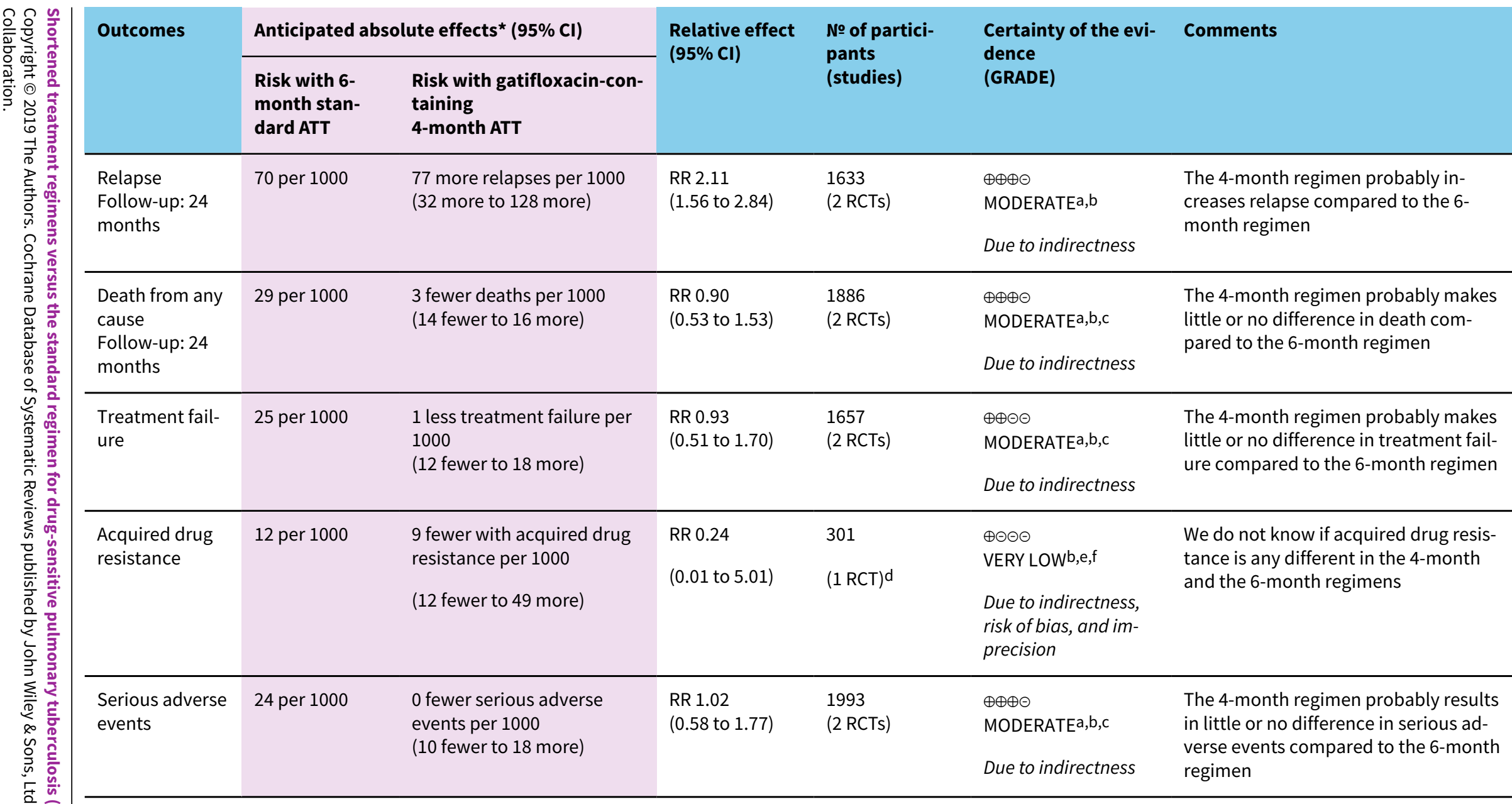

${ }^{\star}$ The risk in the intervention group (and its $95 \%$ confidence interval) is based on the assumed risk in the comparison group and the relative effect of the intervention (and its $95 \% \mathrm{Cl})$.

ATT: anti-tuberculosis treatment; $\mathrm{Cl}$ : confidence interval; RCT: randomized controlled trial; RR: risk ratio.

\section{GRADE Working Group grades of evidence.}

High certainty: we are very confident that the true effect lies close to that of the estimate of the effect.

Moderate certainty: we are moderately confident in the effect estimate: the true effect is likely to be close to the estimate of the effect, but there is a possibility that it is substantially different.

Low certainty: our confidence in the effect estimate is limited: the true effect may be substantially different from the estimate of the effect.

Very low certainty: we have very little confidence in the effect estimate: the true effect is likely to be substantially different from the estimate of effect. 


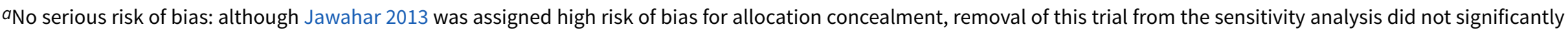
alter the direction, magnitude, or precision of the effect estimate. Not downgraded.

bDowngraded one level for serious indirectness: trials excluded children and adolescents and people with diabetes mellitus and other comorbid physical conditions and those with alcohol abuse.

${ }^{c}$ No serious imprecision: the $95 \% \mathrm{Cl}$ of the risk ratio was wide, but events were few and the sample size was reasonably large; the $95 \% \mathrm{Cl}$ for the absolute estimates did not indicate clinically appreciable benefits for either regimen. Not downgraded.

dOne trial provided data on acquired drug resistance (Jawahar 2013). Merle 2014 reported only drug susceptibility at baseline.

eDowngraded one level for serious risk of bias: allocation concealment was compromised and there were baseline imbalances in proportions with drug resistance at baseline

in the sole trial for this outcome (Jawahar 2013).

fDowngraded two levels for very serious imprecision: the data for acquired resistance come from only one trial with 301 participants, and this trial did not evaluate resistance to gatifloxacin. 


\section{B A C K G R O U N D}

\section{Description of the condition}

Tuberculosis (TB), a chronic infectious disease caused by airborne transmission of aerosolized droplets of Mycobacterium tuberculosis, is a major global public health problem (WHO 2018). An estimated 10 million new cases of tuberculosis and 1.6 million tuberculosis-related deaths occurred in 2017, making tuberculosis one of the top 10 leading causes of death worldwide (WHO 2018). Among the new cases identified, 90\% were adults, 58\% were men, $10 \%$ were children, and $9 \%$ had HIV coinfection (WHO 2018). Among communicable diseases, tuberculosis is a major cause of mortality in the economically productive age group (15 to 49 years) (WHO 2017). The top eight countries in the world identified as having a high tuberculosis burden are India, China, Indonesia, the Philippines, Pakistan, Nigeria, Bangladesh, and South Africa (WHO 2018), and $87 \%$ of tuberculosis occurs in 30 high-burden countries (WHO 2018). To add to the existing burden, 558,000 new cases of rifampicin-resistant tuberculosis were diagnosed in 2017, and of these patients, $82 \%$ had multi-drug resistant tuberculosis (MDRTB) (WHO 2018). Although tuberculosis-related mortality fell by $23 \%$ between 2000 and 2017 worldwide, gaps in diagnosis and treatment persist (WHO 2018).

In May 2014, the World Health Assemby approved The 'End TB Strategy' of the World Health Organization (WHO), which aims to achieve a $95 \%$ reduction in mortality due to tuberculosis and a $90 \%$ reduction in the occurrence of new cases by the year 2035 compared with 2015 estimates (WHO 2015). This can result from a substantial decline in the numbers of tuberculosis cases and deaths in the years to come. However, the rate of decline in the incidence of tuberculosis was 1.9\% from 2015 to 2016; to reach the 'End TB Strategy' targets, this rate of decline must increase to $4 \%$ to $5 \%$ yearly by 2020 . Using the current standard WHO-approved treatment regimen, the treatment success rate for individuals with new and relapsed cases of drug-susceptible tuberculosis, as reported for the 2015 cohort, was $83 \%$ (WHO 2017a). Although this success rate is high when compared with that of individuals with MDR-TB (success rate of 54\%), poor outcomes such as failure to respond, death, and losses to follow-up are of great concern, given that one of the targets of WHO's sustainable development goals for 2030 is to end the global tuberculosis epidemic (WHO 2015; WHO 2018).

The current standard WHO-approved regimen consists of isoniazid, rifampicin, pyrazinamide, and ethambutol (HRZE) for two months (intensive phase), followed by isoniazid and rifampicin with ethambutol (HRE) in areas of high resistance, or without ethambutol (HR) for four months (continuation phase) (WHO 2010). This six-month treatment duration can adversely impact patient adherence to therapy (Zumla 2014). Poor adherence leads to development of drug resistance and enhances the chance of relapse in such individuals (Ginsberg 2010; Ma 2010). Hence, new drug combinations are needed to shorten the course of treatment while maintaining high success rates and low relapse rates. Shortening the duration of treatment for individuals with drug-sensitive or drug-resistant tuberculosis is a global research priority and will certainly be highly beneficial for both patients and healthcare professionals. New tuberculosis drugs have begun to emerge from the clinical development pipeline, and shorterduration regimens containing new compounds could improve adherence to therapy while promoting infection control and leading to better disease management (Ma 2010).

\section{Description of the intervention}

The need for combination therapy for tuberculosis is a result of the distinctive cellular structure of $M$ tuberculosis (a complex array of lipids, proteins, and glycolipids) and the tendency of the bacilli to develop resistance to monotherapy (Kerantzas 2017). Combinations of drugs are required to treat $M$ tuberculosis: combining drugs with both bactericidal activity and sterilizing activity can help target the various bacterial subpopulations (actively dividing, slow growing, and dormant bacilli) present (Mitchison 1985). The bactericidal activity of a drug refers to its ability to kill metabolically active bacilli. An effective bactericidal drug prevents transmission of the bacilli and development of resistance to other drugs given as part of the regimen. The sterilizing activity of a drug refers to its ability to kill all viable bacilli, including the micro-organisms tolerant to treatment with drugs. Drugs with good sterilizing capacity have the potential to shorten the duration of tuberculosis treatment (Ma 2010). In recent years, various drugs have been tried in differing combinations to shorten the standard six-month treatment regimen, and these have shown promising preliminary results (Conde 2011).

Some of the desired characteristics of new anti-tuberculosis drug compounds include the following (Ma 2010).

- Effectiveness against both replicating and dormant tuberculosis bacilli.

- Novel mechanism of action.

- Improved safety profile (versus the standard treatment regimen).

- Good oral bioavailability.

- Low resistance development barrier.

- Minimal interaction with cytochrome p450 enzymes.

- Low cost.

Currently 10 compounds are in clinical development for the treatment of tuberculosis, six have been specifically developed, and four existing drugs have been re-purposed. Drugs at the forefront of this quest include the fluoroquinolones (moxifloxacin, levofloxacin, and gatifloxacin), rifamycins (rifabutin and rifapentine), nitroimidazoles, diarylquinolines, oxazolidinediones, and ethylenediamines. These drugs have been investigated in clinical trials in combination with, or as substitutes for, one of the standard first-line anti-tuberculosis drugs, with the aim of shortening treatment duration (Lienhardt 2010). Second-line antituberculosis drugs, including amoxicillin clavulanate, linezolid, carbapenems, and clofazimine, are also potential candidates for shorter-duration anti-tuberculosis regimens (D'Ambrosio 2015).

\section{How the intervention might work}

\section{Fluoroquinolones}

Fluoroquinolones possess good in vivo and in vitro bactericidal activity against $M$ tuberculosis (Moadebi 2007). This class of drugs acts on the enzyme DNA gyrase, thereby preventing bacterial DNA synthesis (Lienhardt 2010). This mechanism of action is distinct from that of other anti-tuberculosis drugs, raising the possibility of synergistic activity. Overall, the quinolones are well tolerated with minimal side effects on long-term administration (Schluger 2013). 
Fluoroquinolones, when added to an anti-tuberculosis treatment regimen, can enhance the sterilizing and bactericidal effects of combination therapy while increasing drug penetration into chronic tuberculosis lesions. Fluoroquinolones are better tolerated than standard first-line drugs and can shorten treatment duration, hence improving patient adherence to treatment (Ginsburg 2003).

The main concern with quinolones is that they can prolong the QT interval, which may cause ventricular arrhythmias and sudden cardiac arrest (Schluger 2013). The frequency of torsades de pointes - the type of arrhythmia induced by fluoroquinolones - has been reported to be 1 per million with ciprofloxacin or levofloxacin, 3.8 per million with grepafloxacin, and 14.5 per million with sparfloxacin. The chance of arrhythmia is greater for individuals who have associated metabolic disorders such as hypokalaemia or cardiac disease, or who are taking other drugs that can prolong the QT interval (Rubinstein 2002). However, a pooled analysis of data from phase 2, 3, and 4 clinical trials comparing moxifloxacin with other antibiotics showed no clinically relevant differences in cardiac adverse effects between moxifloxacin and comparators (Haverkamp 2012).

\section{Rifamycins}

Rifapentine is a new-generation rifamycin that acts by inhibiting the DNA-dependent RNA polymerase of $M$ tuberculosis. Like other rifamycins, rifapentine can (rarely) cause drug-induced hepatitis and thrombocytopenia (Munsiff 2006). What makes rifapentine a good candidate for tuberculosis therapy shortening and dosage simplification is its long half-life (10 to 15 hours for rifapentine versus two to three hours for rifampicin) and potency against $M$ tuberculosis (Temple 1999). However, compared to rifampicin, rifapentine has poor penetrance into lung cavity lesions, particularly into liquefied caseous material that contains high concentrations of bacteria (Rifat 2018). Consequently, rifapentine requires considerably higher doses than those usually recommended to improve clinical outcomes in pulmonary tuberculosis; some patients with large lung cavitary lesions are less responsive to treatment even with high doses of rifapentine (Savic 2017). Given that currently recommended doses of rifampicin are less effective than higher rifampicin doses in achieving early culture conversion, if higher doses of rifampicin can be shown to reduce relapse rates, this could improve the efficacy of shortened ATT combinations (Boeree 2017).

\section{Nitroimidazoles}

Nitroimidazoles act against both multiplying and dormant bacilli, and thus may be suitable for potentially shortening the duration of tuberculosis therapy ( $M a$ 2010). Two nitroimidazoles are currently being investigated in clinical trials for treatment of individuals with tuberculosis: pretomanid and delamanid. These agents are equally active against drug-sensitive and drug-resistant tuberculosis. They act on the bacilli through bioreduction of the nitroimidazole pharmacophore, generation of reactive oxygen species, and inhibition of mycolic acid synthesis (Matsumoto 2006). In phase 2 trials, QT prolongation was frequently seen in MDRTB patients who received delamanid (Gler 2012). The bactericidal activity of a novel drug combination of pyrazinamide, moxifloxacin, clofazimine, and pretomanid has been compared with that of the standard treatment regimen in individuals with drug-sensitive and drug-resistant tuberculosis. This new regimen was well tolerated and showed greater bactericidal activity than the standard regimen (Dawson 2015).

\section{Diarylquinolines}

One member of this class of drugs - bedaquiline - has been approved as an anti-tuberculosis drug by the European Medicines Agency (EMA) and the US Food and Drug Administration (FDA) (Lessem 2015). Bedaquiline disrupts bacterial metabolism by affecting the synthesis of adenosine triphosphate (ATP) (Andries 2005). The drug is currently used for treatment of MDR-TB, following the findings of a phase 2 trial that demonstrated rapid culture conversion of sputum and low rates of acquired resistance to coadministered drugs (Diacon 2014). Like the quinolones, bedaquiline can cause QT prolongation (Diacon 2012). Bedaqulline has potent late bactericidal properties that exceed those of rifampicin, especially during the second month of therapy, and may have superior sterilizing activity, particularly when combined with pyrazinamide, with the potential to shorten treatment duration for people with drug-sensitive tuberculosis (Andries 2005).

\section{Oxazolidinediones}

Linezolid and sutezolid inhibit the initiation of bacterial protein synthesis by acting on the $50 \mathrm{~S}$ ribosomal subunit. Linezolid, a repurposed drug, is effective in the management of drug-resistant tuberculosis, but adverse effects such as myelosuppression and peripheral neuropathy restrict its long-term use (Sotgiu 2012). A newer addition to this class - sutezolid - is gaining attention, as it has demonstrated greater potency as an anti-tuberculosis drug than linezolid in murine models (Williams 2009). Phase 1 studies in humans have found sutezolid to be safe and well tolerated (Wallis 2010).

\section{Ethylenediamines}

The ethylenediamine, SQ109, inhibits protein synthesis by targeting the membrane transporter, MmpL3, in $M$ tuberculosis, and is effective against drug-susceptible and drug-resistant tuberculosis. In vitro studies showed synergistic effects with bedaquiline and favourable interactions with sutezolid (D'Ambrosio 2015; Sacksteder 2012). However, SQ109 did not shorten time to culture conversion in clinical studies when used in place of ethambutol in anti-tuberculosis regimens (Boeree 2017; Svensson 2018). Further research is required to determine the optimal dose and to identify drug combinations that could optimize the utility of SQ109, if considered for inclusion in treatmentshortening regimens.

\section{Why it is important to do this review}

Novel drug regimens are needed to address the challenges associated with patient adherence to the current standard sixmonth treatment regimen for tuberculosis (Ma 2010). Recent clinical trials have investigated the efficacy of newer regimens administered for less than six months for treatment of individuals with drug-sensitive tuberculosis. A systematic review of these trials will help guide understanding of the efficacy and safety of these shorter regimens among individuals with drug-sensitive pulmonary tuberculosis. A previous Cochrane Review - Gelband 1999 - concluded that longer periods of treatment (at least six months) resulted in higher success rates among individuals with active tuberculosis, but improvement was small when compared with regimens administered for less than six months. Another 
Cochrane Review on the use of fluoroquinolones for treatment of tuberculosis, published in 2013, concluded that evidence was insufficient to support conclusions, but noted that larger trials investigating short-course fluoroquinolone-based regimens were in progress (Ziganshina 2013). First-line treatment with novel drug combinations administered for a shorter duration than the current standard six-month treatment regimen could improve treatment outcomes, thereby reducing the chances of disease transmission and burden in this population.

\section{O B JECTIVES}

To evaluate the efficacy and safety of shortened treatment regimens versus the standard six-month treatment regimen for individuals with drug-sensitive pulmonary tuberculosis.

\section{METHODS}

\section{Criteria for considering studies for this review}

\section{Types of studies}

Randomized controlled trials (RCTs) and quasi-RCTs.

\section{Types of participants}

Newly diagnosed individuals with pulmonary tuberculosis, as defined by positive sputum culture or a positive GeneXpert MTB/ RIF, with presumed or proven drug-sensitive tuberculosis, of all ages, irrespective of HIV status. Trials including people with extrapulmonary tuberculosis were eligible if such participants constituted less than $10 \%$ of participants, or if disaggregated data were available.

\section{Types of interventions}

\section{Intervention}

Treatment regimens of less than six months' duration including any anti-tuberculosis drug(s) or combinations thereof (new drugs or standard anti-tuberculosis drugs at higher than recommended doses).

\section{Control}

Standard first-line therapy for pulmonary tuberculosis, defined as a regimen comprising two months of HRZE and four months of HR or HRE.

\section{Types of outcome measures}

\section{Primary outcomes}

- Relapse of tuberculosis, defined as clinical or bacteriologic recurrence within two years of completion of anti-tuberculosis therapy

\section{Secondary outcomes}

- Death from any cause during anti-tuberculosis therapy or within two years of end of treatment

- Treatment discontinuation: rates of discontinuation of therapy at any time point during treatment

- Positive sputum culture/smear at eight weeks: proportion of participants who remain smear or culture positive at the end of eight weeks of therapy
- Treatment failure: persistent or recurrent positive sputum cultures at the time of treatment completion

- Acquired drug resistance: development of secondary drug resistance to anti-tuberculosis drugs, identified by drug susceptibility testing

\section{Adverse events}

- Serious adverse events: adverse events that were fatal or lifethreatening, or that resulted in a change in treatment regimen

- Other adverse events: other adverse events reported by trial authors, such as hepatitis, prolongation of the QT interval, hypersensitivity reactions, thrombocytopenia, peripheral neuropathy, ocular toxicity, and arthralgia

\section{Search methods for identification of studies}

We attempted to identify all relevant trials regardless of language or publication status (published, unpublished, in press, or in progress).

\section{Electronic searches}

We searched the following databases up to 10 July 2019 using the search terms and strategy we have described in Appendix 1: the Cochrane Infectious Diseases Group Specialized Register; the Central Register of Controlled Trials (CENTRAL), in the Cochrane Library; MEDLINE (PubMed, from 1966); Embase (OVID, from 1947); the Latin American and Caribbean Health Science Information database (LILACS, from 1982); and Science Citation Index-Expanded (Web of Science, from 1900). We also searched the website of the Indian Medlars Center (indmed.nic.in/, 10 July 2019) and the South Asian Database of Controlled Clinical Trials (cochrane-sadcct.org/, 10 July 2019). We searched the WHO International Clinical Trials Registry Platform (who.int/ictrp/en/), ClinicalTrials.gov (clinicaltrials.gov/ct2/home), the Clinical Trials Unit of the International Union Against Tuberculosis and Lung Disease (theunion.org/what-we-do/research/clinical-trials), the UK Medical Research Council Clinical Trials Unit (ctu.mrc.ac.uk/), and Clinical Trials Registry India (ctri.nic.in/) for trials in progress (all accessed on 10 July 2019).

\section{Searching other resources}

We searched the following conference proceedings for abstracts of relevant trials: World Congress on TB, World Lung Conferences of the International Union Against Tuberculosis Lung Disease (2004-2018), American Thoracic Society Meeting Proceedings (2009 to 2019), and the British Society for Antimicrobial Therapy (2010-2019). We contacted relevant organizations, including the Global Partnership to Stop TB and the WHO, for ongoing or completed but unpublished trials. We contacted researchers and experts in the field of clinical trials to identify any additional eligible studies. We checked the references of all included studies to identify additional relevant trials.

\section{Data collection and analysis}

\section{Selection of studies}

Two review authors ( $A G$ and $A M$ ) independently screened all citations and abstracts identified by the search strategy for inclusion. After eliminating duplicates, we scrutinized each report to ensure that multiple publications from the same trial were linked. If eligibility was not clear, or if we noted discrepancies, 
we resolved them through discussion or through consultation with another review author (SJ or JT). AG and AM obtained and scrutinized full texts of potentially eligible studies for inclusion and exclusion. Another review author (PT) independently screened the selected trials and the potentially eligible trials. We listed the excluded studies and tabulated reasons for their exclusion. We presented the study selection process in a PRISMA flow diagram.

\section{Data extraction and management}

Two review authors (AG and AM) independently extracted data using a pre-tested data extraction form. We resolved discrepancies in the extracted data through discussion and by referring to the original articles.

We extracted the following data from the included studies.

- Trial details: publication year, country where the trial was undertaken, study authors, year in which the study was done, study design, number of participants recruited, inclusion criteria, exclusion criteria, recruitment sites.

- Baseline characteristics of participants: age, gender, nutritional status, comorbid illnesses including HIV, sputum smear grading, disease severity, chest X-ray findings.

- Intervention and control arms: numbers allocated to each arm, numbers completing the trial, description of the drugs used in the trial, drug dosage, route and frequency of administration, duration of treatment in the intensive and continuation phases.

- Outcomes: we extracted data for the primary and secondary outcomes as defined above.

For each outcome, we extracted information on the number of participants randomized. For dichotomous outcomes, we extracted the number of participants who experienced the event and the number of people assessed for the event.

Two other review authors (PT and RK) independently verified all extracted data.

\section{Assessment of risk of bias in included studies}

Two review authors ( $A G$ and $A M$ ) independently assessed risk of bias in the trials included in this review using Cochrane's 'Risk of bias' tool in Review Manager 5 (RevMan 5) (Review Manager 2014). We assessed each of the included trials for risk of bias in seven domains: random sequence generation, allocation concealment, blinding of participants and personnel, blinding of outcome assessment (assessed at end of treatment and at end of follow-up), incomplete outcome data, selective outcome reporting, and other potential biases. We resolved disagreements through discussion with a third review author (JT or SS). For each domain in the 'Risk of bias' assessment, we judged the risk of bias as low, high, or unclear. Another review author (PT) independently verified all assessments. We recorded our judgements and support for these judgements in 'Risk of bias' tables accompanying the characteristics of each included study, and we summarized our findings in a 'Risk of bias' summary and graph.

\section{Measures of treatment effect}

All outcomes were dichotomous, and we compared them using risk ratios and presented these with their $95 \%$ confidence intervals.

\section{Unit of analysis issues}

The included studies were parallel-group RCTs. For trials with multiple intervention arms, we undertook pair-wise comparisons of only relevant interventions and when possible combined the results of trial arms with similar ATT regimens. When adverse events were reported as the numbers of events (counts) as well as the numbers of participants experiencing adverse events (rates), we recorded both but used the latter for data synthesis.

\section{Dealing with missing data}

When data for outcomes were missing from the primary trial report, we sought these in supplementary data provided in appendices or related publications. When trials reported intention-to-treat (ITT) or modified intention-to-treat (m-ITT) or per-protocol analyses (available case analyses), we recorded the numbers excluded from analyses from among those randomized and allocated to each arm before and during treatment and during follow-up. We also noted the reasons for their exclusion. Post-randomization exclusions are not uncommon in trials comparing newer ATT regimens. One type occurs when sputum smear positive participants are randomized, but when sputum culture and drug susceptibility results become available, they may not confirm tuberculosis or may indicate infection with other mycobacteria, or the presence of drug resistance. These ineligible participants are excluded from the trials (late screening failures). Modified-ITT analysis in such situations excludes late screening failures from ITT analyses, and all other participants are analysed in their allocated arms. In this deviation from the standard ITT analysis, post-randomization exclusions are unrelated to compliance, withdrawals, or losses to follow-up, or to the likelihood of getting the intervention; when ineligible participants do not represent populations to which trial results are likely to be applied, the risk of bias may not differ from traditional ITT analysis (Fergusson 2002). However, if mITT analyses exclude participants post-randomization for reasons other than late screen failure, this can lead to overestimation of treatment effects compared to standard ITT analyses (Abraha 2015). For this review, we used the data provided in ITT or m-ITT analysis of the included trials for our main analysis, because this analysis included more eligible participants than were included in the reported per-protocol analyses and it did not require us to make assumptions about missing data. When ITT or m-ITT analyses reported in the trials differed from standard interpretations, we assessed the impact of missing data by performing sensitivity analysis for the review's primary outcome of relapse. In imputing missing data, we had intended to perform the commonly used 'best-worst case' analysis, in which the 'best-case' scenario is that all participants with missing outcomes in the experimental intervention group had good outcomes (no relapse), and all those with missing outcomes in the control intervention group had poor outcomes (relapse); the 'worst-case' scenario is the converse. However, these are extreme assumptions, especially with rare outcomes such as relapse. Instead, we used relapse proportions in the treatment and control arms from per-protocol analysis in these trials to impute relapse rates for the missing population.

\section{Assessment of heterogeneity}

We assessed clinical heterogeneity by looking at variability among trial participants, interventions, outcomes, and trial methods, including risk of bias. We assessed statistical heterogeneity by inspecting forest plots for non-overlapping confidence intervals, and we used the $\mathrm{Chi}^{2}$ test with a $10 \%$ level of statistical 
significance to denote that the inconsistency is not due to random error. We used the $\mathrm{I}^{2}$ statistic, with a value of $50 \%$ or greater to generally denote moderate heterogeneity (the proportion of intertrial inconsistency that exceeds random error). However, we acknowledge that absolute thresholds for interpretation of $\mathrm{I}^{2}$ can be misleading. Therefore we interpreted $\mathrm{I}^{2}$ between $0 \%$ and $40 \%$ as possibly unimportant; from $30 \%$ to $60 \%$ as possibly representing moderate heterogeneity; from $50 \%$ to $90 \%$ as representing substantial heterogeneity; and from $75 \%$ to $100 \%$ as showing considerable heterogeneity, depending on the magnitude and direction of effects and the strength of evidence for heterogeneity (e.g. P value from the $\mathrm{Chi}^{2}$ test) (Deeks 2011).

\section{Assessment of reporting biases}

We intended to evaluate the possibility of publication bias by evaluating funnel plots for asymmetry, but because we included fewer than 10 trials, this was not possible.

\section{Data synthesis}

We used risk ratios (RRs) with 95\% confidence intervals (Cls) as summary effect estimates for dichotomous outcomes, and we synthesized data using RevMan 5 (Review Manager 2014). We conducted meta-analyses using a fixed-effect model when heterogeneity was low and a random-effects model when heterogeneity was moderate (see Assessment of heterogeneity section). However, if heterogeneity was moderate and inconsistency was due to trials with large and small effects favouring an intervention, this need not necessarily denote imprecision of clinical importance (Guyatt 2011c). In such instances, if using a random-effects model in sensitivity analyses also resulted in $95 \% \mathrm{Cls}$ indicating appreciable effects of the intervention (see Sensitivity analysis), we used the fixedeffect model in meta-analysis but also reported random-effects estimates in the results. If random-effects meta-analysis had resulted in imprecision (as indicated by the $95 \% \mathrm{Cl}$ including non-appreciable benefits) or had changed the direction of effect, we would have retained the random-effects model in metaanalysis. If heterogeneity was substantial but could be explained in subgroup analyses (see below), we provided effect estimates for the subgroups without an overall pooled effect estimate.

\section{Certainty of the evidence}

We assessed the certainty of evidence by using the GRADE approach for the primary outcome of relapse and for the secondary outcomes important for clinical decision-making, that is, death due to any cause, treatment failure, development of drug resistance, and serious adverse events (Guyatt 2011a). For each of these outcomes, we assessed how certain we were that pooled effect estimates were true (Balshem 2011), and that their $95 \%$ Cls represented the range of effects that were plausible and likely to be useful (Hultcrantz 2017). Certainty of evidence for each outcome is influenced by risk of bias in the studies contributing to pooled effect estimates for each outcome, as well as other factors such as unexplained inconsistency, indirectness, imprecision, and publication bias (Balshem 2011). Pooled effect estimates from RCTs are generally considered to provide high-certainty evidence, but if there were serious or very serious concerns that any of the above-mentioned factors may have compromised the certainty of effect estimates, we rated down the certainty for that outcome by one or two levels. In making these assessments, we used the overall guidance provided in Schünemann 2011 and Schünemann 2013. We also used guidance provided in Guyatt 2011b to assess the impact of imprecision on the certainty of evidence for each outcome. According to this guidance, precision is considered adequate if the $95 \% \mathrm{Cl}$ excludes an RR of 1.0, and the total number of events or patients in the total sample size is large enough to satisfy or exceed that required for an adequately powered individual trial (optimal information size, or OIS). However, when event rates are very low, as is likely with trials comparing shortened versus standard ATT regimens that were designed to assess equivalence or non-inferiority within prespecified non-inferiority margins, $\mathrm{Cls}$ around relative effects may be wide but $\mathrm{Cls}$ around absolute effects will be narrow. In such instances, rating down for imprecision may be inappropriate (Guyatt 2011b). For rating inconsistency, we used guidance provided in Guyatt 2011c, particularly when heterogeneity was moderate in fixed-effect meta-analysis but inconsistency in results was due to trials with large and small effects favouring an intervention. In such instances, if using a randomeffects model did not result in $95 \% \mathrm{Cls}$ that now included nonappreciable effects or no benefit associated with the intervention, we did not rate down for imprecision. We incorporated the ratings on certainty of evidence for effect estimates for each outcome along with relative and absolute measures of effect in 'Summary of findings' tables for each comparison in this review, using the GRADEpro Guideline Development Tool (GRADEpro GDT).

\section{Subgroup analysis and investigation of heterogeneity}

When we considered heterogeneity to be moderate or substantial, we explored potential causes in subgroup analyses based on categories of shortened treatment regimens. We subgrouped fourmonth regimens according to whether they replaced components of standard ATT drugs or augmented them in comparison with standard six-month ATT regimens.

\section{Sensitivity analysis}

We re-analysed data using a random-effects model in sensitivity analysis if fixed-effect meta-analysis revealed moderate heterogeneity but inconsistency in results of the trials was due to differences in the magnitude of effect favouring an intervention, rather than to differences in the direction of effects. Moderate inconsistency need not necessarily reduce our confidence in the pooled estimate if inconsistency is largely due to differences between large and small effects favouring an intervention (Guyatt 2011c). Thus, when we judged heterogeneity to be moderate but inconsistency in results was due to large and small effects favouring an intervention, we assessed the robustness of the results by changing from a fixed-effect to a random-effects metaanalysis. If pooled effect estimates in random-effects meta-analysis continued to favour the intervention, and if both limits of the $95 \% \mathrm{Cl}$ continued to indicate appreciable benefit, we used the fixed-effect model in the analysis but reported both fixed-effect and random-effects meta-analysis in the results. We retained the fixed-effect model in meta-analysis in such instances to avoid compromising grading of imprecision in evaluating certainty of the evidence while summarizing the findings. Random-effects metaanalyses provide pooled estimates of the range of possible effects, with point estimates representing the mean of their distribution; this inherently denotes imprecision. Using the random-effects model under such circumstances would warrant rating down for imprecision while assessing the certainty of evidence when this is not warranted. 
We also assessed the impact of risk of bias on effect estimates of the primary outcome in sensitivity analysis by excluding studies judged to be at high risk of bias. We explored the effects of missing outcome data for the primary outcome of relapse in sensitivity analysis comparing results of the main analysis with results of the per-protocol analysis, and including all randomized participants (excluding late screening failures, treatment failures, and deaths), and we imputed missing data using relapse rates from available data.

\section{RE S U LTS}

\section{Description of studies}

\section{Results of the search}

We identified 1489 articles through database screening and two articles by searching other sources. After screening the 1491 titles and abstracts, we excluded 1457 records that were not relevant. We retrieved 34 full-text records of potentially eligible studies (Figure 1). We excluded 11 records of RCTs that did not fulfil the inclusion criteria for the review (see Characteristics of excluded studies). We identified 23 relevant records for inclusion that reported on 11 RCTs. Eight of these records pertained to six ongoing studies that are detailed in Characteristics of ongoing studies. The remaining 15 records related to five RCTs that met criteria for selection to this review. No studies await assessment.

Figure 1. Study flow diagram.

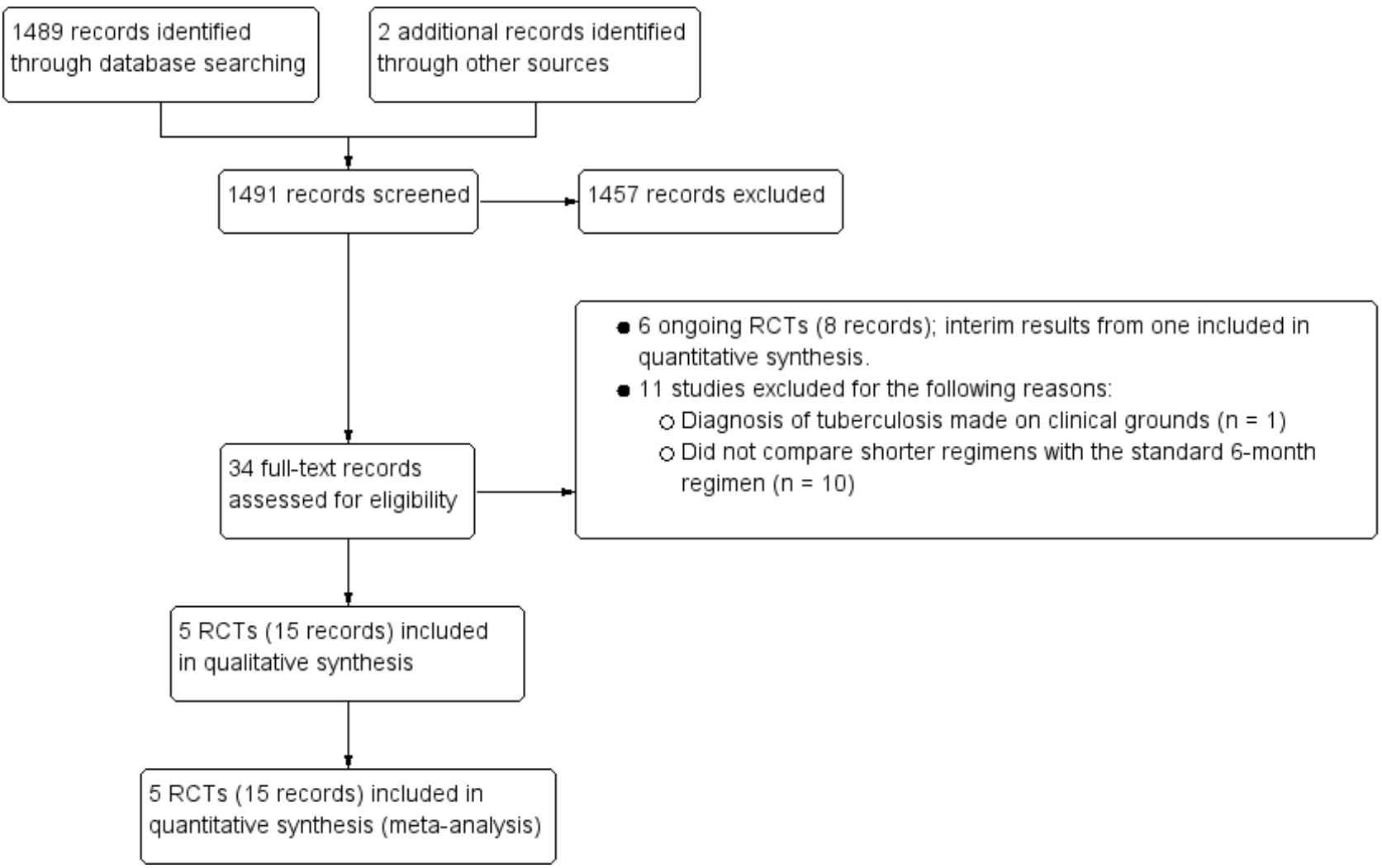

\section{Included studies}

We included five RCTs that randomized a total of 5825 participants (Gillespie 2014; Jawahar 2013; Jindani 2014; Merle 2014; Velayutham 2014). Refer to Characteristics of included studies for a summary of included trial characteristics. Table 1 provides additional descriptive details.

\section{Setting}

Three of the included trials were multi-country trials. Gillespie 2014 (REMoxTB study) included participants from multiple sites in nine countries: four in Africa (Kenya, South Africa, Tanzania, Zambia), four in Asia (China, India, Malaysia, Thailand), and one in Latin America (Mexico). Jindani 2014 (RIFAQUIN trial) recruited participants from six cities in four countries in Africa (Botswana, South Africa, Zambia, Zimbabwe). Merle 2014 (OFLOTUB/Gatifloxacin) included participants from five cities in five countries in Africa (Benin, Guinea, Kenya, Senegal, South Africa). The other two trials were conducted in two cities in south India (Jawahar 2013; Velayutham 2014).

\section{Study participants}

The five trials recruited only adults ( $>18$ years of age). Most participants were male, ranging from $64 \%$ to $74 \%$ across the five trials. Two trials excluded HIV-positive participants (Jawahar 2013; Velayutham 2014). Gillespie 2014 included 110 HIV-positive participants ( $7 \%$ in each arm) whose CD4 counts were $>250$ cells/ $\mu \mathrm{L}$, and who were not receiving antiretroviral treatment (ART). 
Merle 2014 included 304 (18.1\%) individuals with HIV who were not in stage 3 or 4 disease and were not receiving ART (17.4\% in the shortened regimen, $18.7 \%$ in the standard regimen). Jindani 2014 included the largest proportion of HIV-positive participants (158; $27 \%$ ) after excluding those with CD4 count $<150 / \mathrm{mm}^{3}$ and those on ART; $28 \%$ were allocated to the shortened regimen and $29 \%$ to the six-month regimen.

All five trials included patients with lung cavitation. In Gillespie 2014 , this accounted for $71 \%$ overall $(69 \%$ and $70 \%$ in the intervention groups, $72 \%$ in the control group). In Jindani 2014, $67 \%$ given the control regimen and $65 \%$ receiving the shortened regimen had cavitation. Merle 2014 included 50\% in the control regimen and $52 \%$ in the shortened regimen with cavitation. Velayutham 2014 reported that cavitation was present in $36 \%$ of those allocated to the shortened regimen and in $41 \%$ of those given the control regimen. Jawahar 2013 did not provide numerical data about proportions with lung cavitation.

Gillespie 2014 and Jindani 2014 excluded those with body weight less than $35 \mathrm{~kg}$; in Gillespie 2014 and in Jindani 2014, 9\% to 11\% and $4 \%$ to $5 \%$ of included participants, respectively, had body weight $<40$ kg. Jawahar 2013 and Velayutham 2014 excluded participants who weighed $<30 \mathrm{~kg}$. In Jawahar 2013, mean body weight ranged from $43.7 \mathrm{~kg}$ to $44.2 \mathrm{~kg}$ in the shortened treatment arms and was 43 $\mathrm{kg}$ in the control arm. In Velayutham 2014, 53\% in the shortenedtreatment arms and 54\% in the standard treatment arm weighed > $42 \mathrm{~kg}$. Merle 2014 required participants to weigh between $38 \mathrm{~kg}$ and $80 \mathrm{~kg}$; mean weight was $53.8 \mathrm{~kg}$ in the intervention arm and $54.2 \mathrm{~kg}$ in the control arm.

The diagnosis was made by using two positive sputum samples and was confirmed by culture in all trials. Gillespie 2014 required culture-confirmed susceptibility to rifampicin, isoniazid, pyrazinamide, and moxifloxacin; Jindani 2014 additionally required susceptibility to isoniazid; and Merle 2014 required susceptibility to ethambutol and gatifloxacin. All trials excluded people with MDR-TB (Table 1).

\section{Shorter ATT regimens}

The five included studies evaluated shorter regimens involving two fluoroquinolones (moxifloxacin and gatifloxacin) given to 3512 participants compared to 2176 participants given standard six-month ATT regimens. We did not find trials evaluating other fluoroquinolones, nitroimidazoles, diarylquinolines, oxazolidinediones, or ethylenediamines in shortened ATT regimens compared to standard ATT regimens. We also did not find eligible trials that included other candidate drugs for shorter regimens, such as amoxicillin clavulanate, linezolid, carbapenems, or clofazimine.

\section{Comparision 1. Moxifloxacin-containing four-month ATT regimens}

Four trials compared moxifloxacin-containing shortened ATT regimens (three to four months) versus standard six-month ATT regimens.They differed in whether moxifloxacin was used to replace one of the standard ATT drugs in the four-month ATT arm (Gillespie 2014; Jawahar 2013; Jindani 2014), or to augment them (Velayutham 2014). Treatments were supervised in all trials.

\section{Moxifloxacin replacing standard ATT drugs}

Gillespie 2014 (REMoxTB study) randomized 1931 participants to three arms. Two arms compared moxifloxacin-containing daily regimens for four months ( 17 weeks) versus a control intervention for six months ( 26 weeks) of a daily ATT regimen. One arm (isoniazid group, where moxifloxacin (M) (400 mg) replaced ethambutol (E); $N=655)$ received eight weeks of $M$ with isoniazid, rifampicin, and pyrazinamide (HRZ) plus E placebo administered daily, followed by nine weeks of MHR, followed by nine weeks of $\mathrm{H}$ and $\mathrm{R}$ placebo. The second intervention arm (ethambutol group, where moxifloxacin $(400 \mathrm{mg}$ ) replaced isoniazid; $\mathrm{N}=636$ ) received eight weeks of MRZE plus $\mathrm{H}$ placebo administered daily, followed by nine weeks of MR plus $\mathrm{H}$ placebo daily, followed by nine weeks of $\mathrm{H}$ and $\mathrm{R}$ placebo. The control arm $(N=640)$ received eight weeks of HRZE and $M$ placebo given daily, followed by nine weeks of HR and M placebo given daily, followed by nine weeks of HR. Results of the two moxifloxacin arms did not differ significantly. We combined the data for these two intervention arms compared to the six-month regimen in data synthesis for our primary analysis.

Jawahar 2013 randomized 429 participants to three arms. In the two intervention arms, gatifloxacin $(G)$ or moxifloxacin $(M)$ replaced ethambutol in the shortened regimen. The moxifloxacin arm $(\mathrm{N}=$ 118 ) received two months of moxifloxacin $(400 \mathrm{mg})$ and $\mathrm{HRZ}$ thrice weekly, followed by two months of MHR thrice weekly. The control arm $(N=170)$ received two months of HRZE thrice weekly, followed by four months of HR thrice weekly. This trial was stopped early by the data safety monitoring board at a planned interim analysis, after it had recruited only a third of the 1200 estimated sample, due to higher relapse rates in the intervention arms.

Jindani 2014 (RIFAQUIN trial) also had three arms randomizing 827 participants (of the estimated sample size of 1095). In two intervention arms, moxifloxacin $(400 \mathrm{mg})$ replaced isoniazid throughout, and high-dose $(900 \mathrm{mg})$ rifapentine $(\mathrm{P})$ replaced rifampicin in the continuation phase. We did not include one of these arms in data synthesis because the four-month continuation phase resulted in a six-month ATT regimen. In the other arm, 275 participants were given eight weeks of MRZE administered daily, followed by nine weeks of MP administered twice weekly. In the control arm, 275 participants were given eight weeks of HRZE administered daily, followed by 18 weeks of HR daily.

\section{Moxifloxacin augmenting standard ATT drugs}

Velayutham 2014 is the interim report of an ongoing trial CTRI/2008/091/000024 - that compared four different regimens in which moxifloxacin $(400 \mathrm{mg}$ ) was added to HRZE in shortened courses. The four arms randomized 629 participants to receive HRZEM daily for three months, or daily for two months followed by RHM daily for two months, or daily for two months followed by RHM thrice weekly for two months, or daily for two months followed by RHEM thrice weekly for two months. The standard six-month (2HRZE/4HR) regimen was given thrice weekly to 172 participants. The report presented planned interim outcomes and final results are awaited.

\section{Comparison 2. Gatifloxacin-based four-month ATT regimens}

\section{Gatifloxacin replacing standard ATT drugs}

Merle 2014 (OFLOTUB/gatifloxacin) randomized 1836 participants, of whom 917 were given two months of gatifloxacin $(400 \mathrm{mg}$; replacing ethambutol) and HRZ daily, followed by two months of daily HRG. In the control arm, 919 participants were given the standard daily six-month (2HRZE/4HR) regimen. 
In Jawahar 2013, the gatifloxacin arm replaced ethambutol in 141 participants who received two months of HRZG thrice weekly, followed by two months of HRG thrice weekly. The 170 participants in the control arm received $2 \mathrm{HRZE} / 2 \mathrm{HR}$ given thrice weekly.

\section{Follow-up}

Participants in three of the included trials were followed for a period of 24 months after end of treatment (Jawahar 2013; Merle 2014; Velayutham 2014). Gillespie 2014 and Jindani 2014 followedup participants for a period of 18 months after randomization (12 months after treatment). However, $14 \%$ of participants in Jindani 2014 who were randomized in the last six months of enrolment received follow-up for 12 or 15 months after randomization. All trials reported regular scheduled assessments for efficacy and safety outcomes for participants in the intervention and control arms (see Characteristics of included studies).

\section{Outcomes}

Four trials provided data on relapse - the primary outcome of this review (Gillespie 2014; Jawahar 2013; Jindani 2014; Merle 2014). In Gillespie 2014 and Jindani 2014, relapse was differentiated from re-infection through genotyping of patients with culture-confirmed recurrence. In Merle 2014, genotyping results were available for only 77 of $140(55 \%)$ of those with culture-confirmed recurrence. However, $79 \%$ of the 77 with genotyping results were confirmed as relapses. In Jawahar 2013, relapse was not differentiated from re-infection but most recurrences occurred within six months after treatment, suggesting that these were instances of relapse.

Again, four trials provided data on death from any cause, including tuberculosis, that occurred on treatment and during follow-up (Gillespie 2014; Jawahar 2013; Jindani 2014; Merle 2014). No deaths were reported in the interim analysis provided in Velayutham 2014. Rates of treatment discontinuation and treatment failure were reported in four trials (Gillespie 2014; Jawahar 2013; Jindani 2014; Merle 2014), with different definitions used to compute these outcomes (Table 1 ).

Four trials reported the outcome of sputum culture positivity at eight weeks (Gillespie 2014; Jawahar 2013; Merle 2014; Velayutham 2014). In Velayutham 2014, data for this outcome were presented for all participants allocated to four groups combined, but because participants in the four groups had received identical regimens for the first two months, we used these data in the meta-analysis. In the fifth trial (Jindani 2014), these results were presented as combined data for the four-month and six-month moxifloxacin arms, and disaggregated data for sputum positivity at two months were not available. Gillespie 2014, Jawahar 2013, and Jindani 2014 provided data on acquired drug resistance. Merle 2014 and Velayutham 2014 did not report on this.
Acquired drug resistance was assessed and reported in three trials (Gillespie 2014; Jawahar 2013; Jindani 2014), which assessed drug susceptibility at baseline as well as in those who were culture positive at end of treatment, or who experienced relapse/ recurrence. Resistance results were missing for isoniazid in 24 patients and for pyrazinamide in 27 patients at baseline in Gillespie 2014, and the cases of acquired drug resistance reported were only probable and were not unequivocal in the absence of whole genome sequencing. Jawahar 2013 did not directly assess susceptibility to moxifloxacin and gatifloxacin but used susceptibility to ofloxacin as a proxy indicator. Merle 2014 assessed drug susceptibility at baseline and performed indirect drug susceptibility tests during follow-up but did not report acquired drug resistance.

Serious adverse events experienced by trial participants were reported in all trials or could be deduced from the adverse events reported. Gillespie 2014 and Jindani 2014 did not report adverse events other than serious adverse events. Merle 2014 also reported the proportions of participants with QT prolongation and with hyperglycaemic episodes.

\section{Excluded studies}

We excluded 11 studies for reasons detailed under Characteristics of excluded studies. One trial, Alavi 2009, studied the effects of rifampicin, isoniazid, and ofloxacin in people with smear negative pulmonary tuberculosis, diagnosed solely on the basis of clinical criteria. Five were phase $2 \mathrm{~b}$ trials with no six-month standard ATT comparator arm (Burman 2006; Conde 2009; Conde 2016; Dorman 2009; Rustomjee 2008). These trials, along with El-Sadr 1998 which we excluded because it compared levofloxacin added for the first two months of the standard six-month ATT regimen versus six to nine months of standard ATT regimens, are included in an earlier Cochrane Review (Ziganshina 2013). We excluded three other trials because they lacked comparisons with a standard six-month ATT arm (Kohno 1992; Tuberculosis Research Centre 1986; Tuberculosis Research Centre 2002). Johnson 2009 evaluated the effects of four months of standard ATT drugs versus six months of standard ATT but randomized only those who were sputum negative after four months of treatment to receive no further treatment or two more months of ATT.

\section{Risk of bias in included studies}

Please refer to Figure 2 for the summary of 'Risk of bias' assessments for each included study, and to Figure 3 for a risk of bias graph regarding each item presented as percentages across all included trials. Please also see 'Risk of bias' tables for individual trials under Characteristics of included studies for supporting evidence on the judgement of risk of bias for the included studies. 
Figure 2. Risk of bias summary: review authors' judgements about each risk of bias item for each included trial.

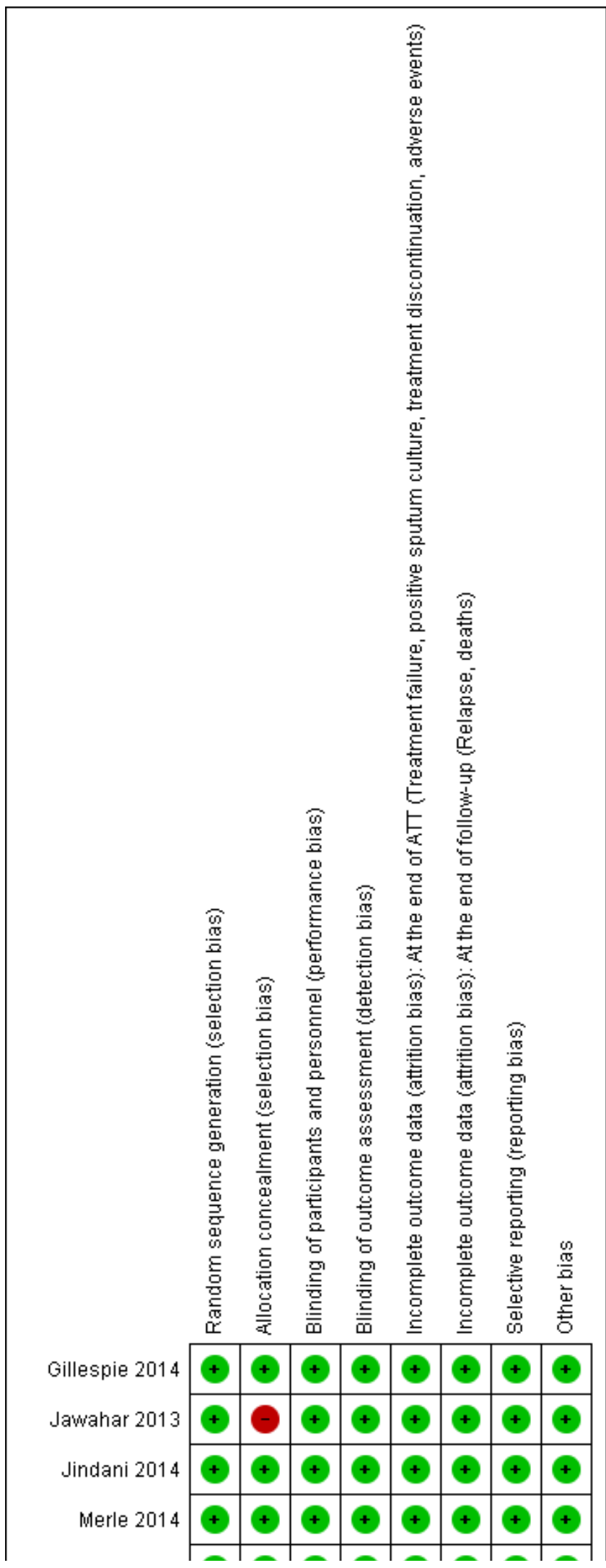


Figure 2. (Continued)

\begin{tabular}{|c|c|c|c|c|c|c|c|c|}
\hline Merle 2014 & + & + & + & + & + & + & + & + \\
\hline Velayutham 2014 & + & + & + & + & + & + & + & + \\
\hline
\end{tabular}

Figure 3. Risk of bias graph: review authors' judgements about each risk of bias item presented as percentages across all included studies.

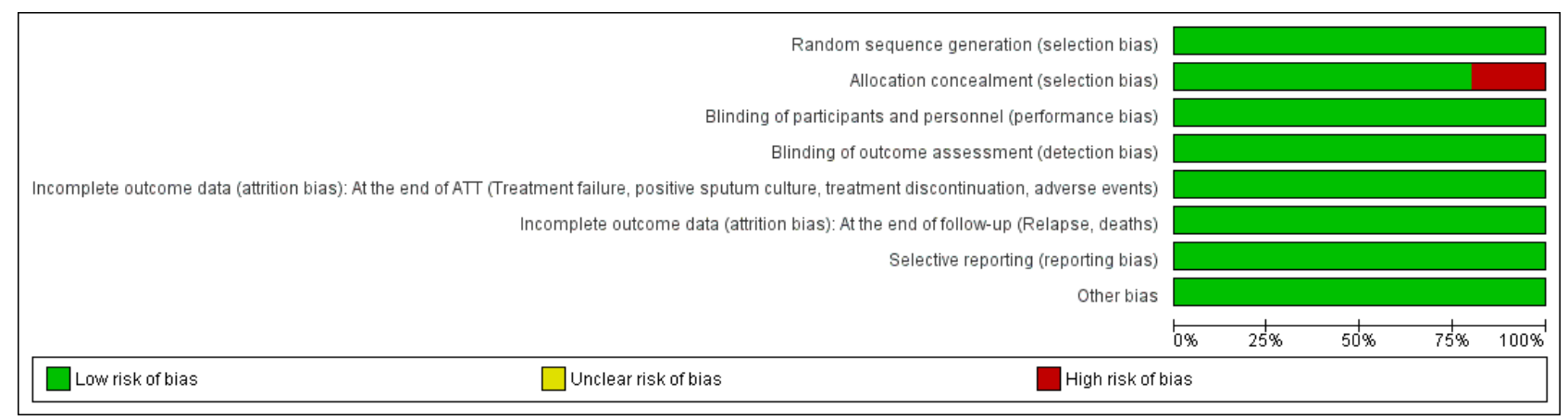

\section{Allocation}

All included studies were judged to be at low risk of bias for generating the random sequence. All but one - Jawahar 2013 were judged as having low risk of bias for allocation concealment. Jawahar 2013 was judged to be at high risk of bias because recruitment ratios were altered during the course of the trial, thus likely compromising concealment of allocation. In conjunction with premature termination of the trial following a planned interim analysis, this led to baseline imbalance in some prognostic indicators.

\section{Blinding}

In Jawahar 2013, participants and care providers were not blinded to the interventions, and allocation concealment was likely to have been compromised. Jindani 2014 was an open-label trial and treating physicians were aware of the treatment allocated. However, we believe this did not increase the risk of performance bias in these trials because we found no evidence that this influenced the administration of interventions or co-interventions differentially between four-month and six-month regimens. We judged the other three trials to have low risk of performance bias, and we judged the five included trials as having low risk for detection bias.

\section{Incomplete outcome data}

We judged the five trials to be at low risk of attrition bias for outcomes assessed at the end of ATT and at the end of follow-up. These trials had low attrition (Jawahar 2013; Velayutham 2014), or, if attrition exceeded 10\% (Gillespie 2014; Jindani 2014; Merle 2014), differential attrition was not substantial and the results of per-protocol analysis, modified intention-to-treat analyses, and other sensitivity analyses reported in the trials were consistent. In Jawahar 2013, although the power of the trial to prove equivalence was reduced due to early termination, we judged this study as having low risk of attrition bias, as attrition was low with similar reasons for exclusion, and this was unlikely to have altered the relative estimates of effects.

\section{Selective reporting}

The five studies reported all outcomes stated in the methods sections of their trial publications, or their protocols, or their clinical trial registry documents, and we judged them to be at low risk of reporting bias.

\section{Other potential sources of bias}

In three trials (Gillespie 2014; Jindani 2014; Merle 2014), study drugs were provided by their manufacturers, but we judged these studies to be at low risk of bias because the trial publications provided explicit statements that the manufacturers had no role in the study nor in the publication of results. We did not detect any other sources of bias.

\section{Effects of interventions}

See: Summary of findings for the main comparison Moxifloxacincontaining 4-month ATT regimens versus standard 6-month ATT regimen for drug-sensitive pulmonary tuberculosis; Summary of findings 2 Gatifloxacin-containing 4-month ATT regimens compared to standard 6-month ATT regimens for drug-sensitive pulmonary tuberculosis

\section{Comparison 1. Moxifloxacin-containing four-month ATT regimens versus standard six-month ATT regimens}

\section{Primary outcome}

Relapse

Three trials provided data on relapse over 12 to 24 months following treatment in people with drug-sensitive pulmonary tuberculosis (Gillespie 2014; Jawahar 2013; Jindani 2014). Two trials differentiated relapse from re-infection using molecular methods (Gillespie 2014; Jindani 2014). Of 2769 participants randomized to the three regimens, 2265 participants (82\%) were culture negative at the end of treatment and were evaluated for relapse or recurrence. Relapse proportions for the two regimens compared in the three trials are shown in Table 2. 
Overall, $177(5.2 \%)$ in the two groups included in the primary modified ITT analysis experienced a recurrence; most cases (156/178; 88\%) were confirmed as relapse through genotyping; and 17 of $21(81 \%)$ tuberculosis recurrences in Jawahar 2013 occurred in the first six months after treatment, suggesting that they were relapses rather than re-infections. Relapse in the six-month ATT arm varied from $2.3 \%$ of 555 participants in Gillespie 2014, to $6.5 \%$ of 155 participants in Jawahar 2013 and $3.7 \%$ of 163 participants in Jindani 2014. The corresponding incidence of relapse in the moxifloxacin-containing shorter ATT regimens was $9.8 \%$ of 1119 in Gillespie 2014, $10.1 \%$ of 108 in Jawahar 2013, and $16.4 \%$ of 165 in Jindani 2014. Meta-analysis showed that risk of relapse was thrice more common with moxifloxacin-containing four-month ATT regimens than with the standard six-month regimen (RR 3.56, 95\% Cl 2.37 to 5.37; 2265 participants, 3 trials; Analysis 1.1). Results showed some heterogeneity $\left(\mathrm{I}^{2}=54 \%\right)$, but inconsistency between large and small effects favoured the six-month ATT regimen, with considerable overlap in the $95 \% \mathrm{Cl}$ of the effect estimates. Reanalysing data in sensitivity analysis using a random-effects model did not introduce imprecision into estimates of appreciable benefit with the six-month regimen (RR 3.18, 95\% $\mathrm{Cl} 1.69$ to 5.97).

In the main analysis, we combined modified-ITT data from the two moxifloxacin-containing intervention arms in Gillespie 2014. We subgrouped data according to whether moxifloxacin was used in the four-month regimen to replace ethambutol in the intensive phase (one of the intervention arms in Gillespie 2014 and the moxifloxacin arm in Jawahar 2013), or to replace isoniazid in the four-month regimen (the other intervention arm in Gillespie 2014, and the moxifloxacin with high-dose rifapentine arm in Jindani
2014). Results again favoured the six-month ATT regimen (Analysis 1.2), irrespective of whether moxifloxacin replaced isoniazid in fourmonth ATT regimens (RR 2.74, 95\% $\mathrm{Cl} 1.69$ to 4.43; 747 participants, 3 trials; Analysis 1.2: subgroup 1), or whether moxifloxacin replaced ethambutol (RR 4.89, 95\% Cl 3.02 to $7.92 ; 1424$ participants, 2 trials; Analysis 1.2: subgroup 2). We did not undertake subgroup analysis based on HIV status as there were only three trials, and one excluded HIV-positive people (Jawahar 2013). However, Gillespie 2014 and Jindani 2014 reported no significant interaction effects between HIV status and unfavourable outcomes in subgroup analyses.

Jawahar 2013 was at high risk of bias for allocation concealment, and we explored the impact of this in sensitivity analysis by removing this study's data. Pooled estimates from the two studies without high risk of bias also show that the four-month regimen increases relapse compared to the standard six-month regimen (RR $4.26,95 \% \mathrm{Cl} 2.65$ to $6.84 ; 2002$ participants, 2 trials; $I^{2}=0 \%$ ).

We used data for relapse from m-ITT analyses reported in the three trials for the main meta-analysis in this review. However results did not differ substantially when we performed sensitivity analyses using data from the per-protocol analyses in the three trials in metaanalysis (Table 2; Analysis 1.3: subgroup 2). When we explored the impact of missing data for all randomized participants (excluding late screening failures, treatment failures, and deaths) and imputed relapse rates for missing participants using relapse proportions reported in per-protocol analyses of individual trials, results were consistent with the main meta-analysis (Table 2; Analysis 1.3: subgroup 3; Figure 4).

Figure 4. Forest plot of comparison: 1 Moxifloxacin-containing 4-month ATT versus standard 6-month ATT regimens, outcome: 1.3 Relapse: sensitivity analysis accounting for missing data.

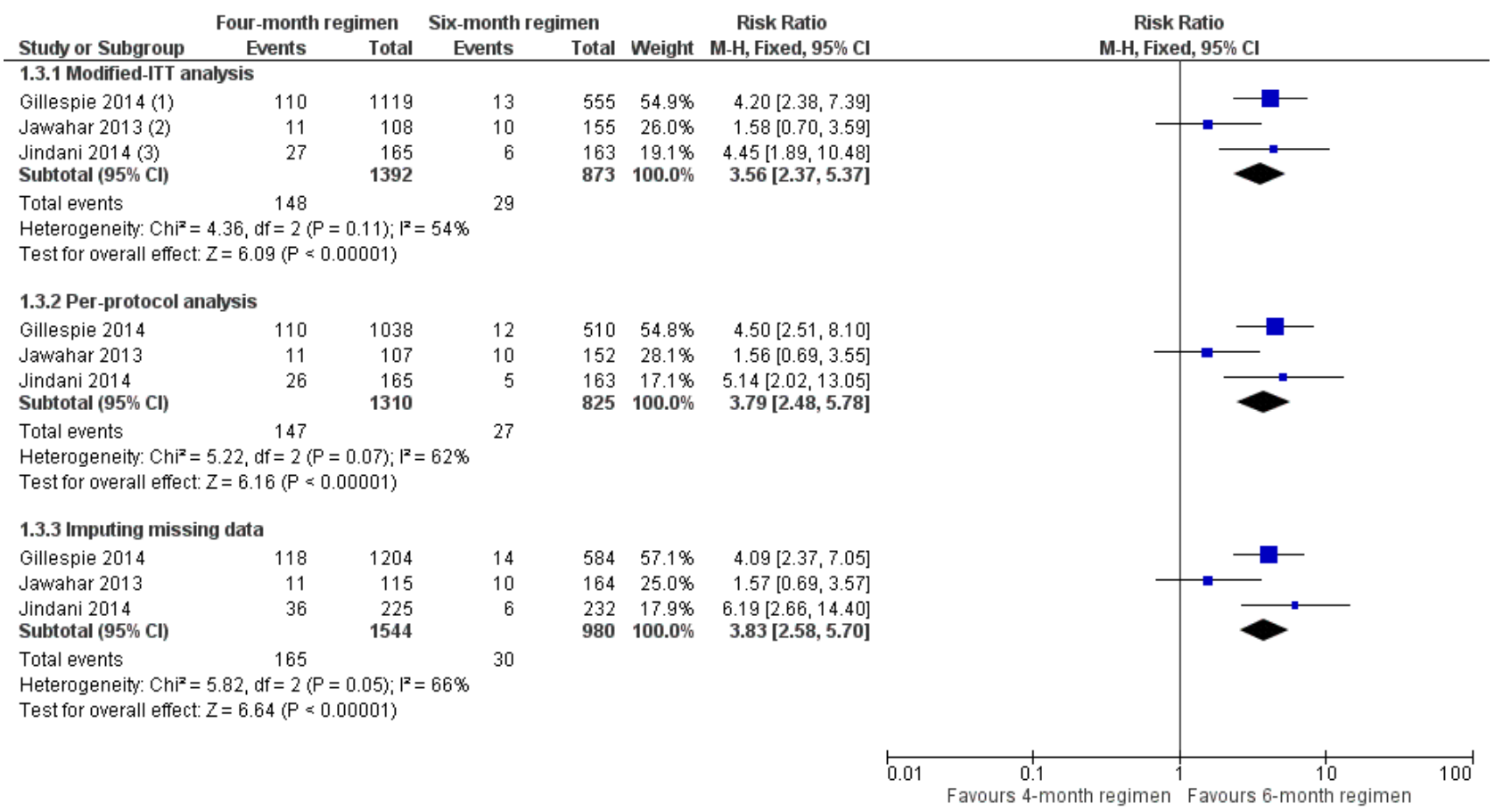

Footnotes

(1) Moxifloxacin daily for 4 months replacing isoniazid (or ethambutol in first 2 month) versus daily standard 6-month regimen; FU: 12 months after treatment

(2) Moxifloxacin thrice-weekly for 4 months (replacing ethambutol) versus thrice-weekly standard 6-month regimen; FU: 24 months after treatment

(3) Moxifloxacin replacing isoniazid (daily for 2 months + twice-weekly rifapentine for 2 months) versus standard 6 -month daily regimen; FU:18 months after treatment 


\section{Secondary outcomes}

\section{Death from any cause}

Three trials reported 62 deaths (Gillespie 2014; Jawahar 2013; Jindani 2014). Gillespie 2014 reported 27 deaths with four-month ATT; 19 (70\%) were tuberculosis-related deaths, and 11 of 16 (69\%) deaths with six-month ATT were tuberculosis-related. The one death (non-tuberculosis) in Jawahar 2013 occurred with six-month ATT. Jindani 2014 reported 2 of 12 (16\%) deaths as tuberculosis-related with the four-month regimen, and 1 of 6 $(16 \%)$ as tuberculosis-related deaths with six-month ATT. Pooled estimates of the risk of death due to any cause did not significantly differ between four-month and six-month ATT regimens (2760 participants, 3 trials; Analysis 1.4).

\section{Treatment discontinuation}

Of 2335 evaluable participants in three trials (Gillespie 2014; Jawahar 2013; Jindani 2014), 121 (5.2\%) discontinued treatment for different reasons (Table 1). In meta-analysis, treatment discontinuation showed little or no difference between the two groups (2335 participants, 3 studies; Analysis 1.5).

\section{Sputum culture/smear positivity at eight weeks}

Data for sputum culture conversion at the end of the intensive phase of ATT treatment were reported by all trials in the review; however, Jindani 2014 reported only combined sputum conversion data for the four-month and six-month moxifloxacin-containing ATT arms of the trial. Disaggregated data for the four-month moxifloxacin arm were not available for inclusion in meta-analysis.

The pooled point estimate from the three trials with usable data for sputum culture/smear positivity at eight weeks favoured the four-month moxifloxacin-containing ATT regimen (Gillespie 2014; Jawahar 2013; Velayutham 2014), but the $95 \% \mathrm{Cl}$ did not rule out a small benefit for the standard six-month ATT regimen, and heterogeneity was substantial $\left(\mathrm{I}^{2}=91 \% ; 2828\right.$ participants, 3 trials; Analysis 1.6). We explored heterogeneity by subgrouping the data according to whether moxifloxacin replaced isoniazid or ethambutol in four-month ATT regimens (Gillespie 2014; Jawahar 2013), or augmented standard ATT drugs in four-month ATT regimens in random-effects meta-analysis(Velayutham 2014). Fourmonth moxifloxacin-containing regimens that replaced isoniazid or ethambutol were not unequivocally better than standard sixmonth ATT regimens in achieving sputum culture conversion at eight weeks (2087 participants, 2 trials; Analysis 1.6: subgroup 1). However, moxifloxacin augmentation of standard ATT drugs in four-month regimens was more effective than standard sixmonth ATT in sterilizing sputum (sputum positivity at eight weeks $4.6 \%$ versus $19.2 \%$; RR $0.24,95 \% \mathrm{Cl} 0.15$ to $0.39,741$ participants; Analysis 1.6: subgroup 2) (Velayutham 2014). The test for subgroup differences confirmed that moxifloxacin augmentation rather than substitution of standard ATT drugs achieves better sputum conversion at eight weeks compared to standard six-month ATT regimens $\left(P=0.001 ; I^{2}=90.2 \%\right.$; Analysis 1.6).

\section{Treatment failure}

In the three trials that reported this outcome (Gillespie 2014; Jawahar 2013; Jindani 2014), treatment failures were equally rare, with only 14 failures reported among 1399 participants evaluated in the four-month arm and 14 among 883 participants evaluated in the six-month arm (2282 participants, 3 trials; Analysis 1.7). Most of these were culture confirmed treatment failures.

\section{Acquired drug resistance}

Acquired drug resistance was evaluated in three of the four included trials among those who had treatment failure, or who suffered a relapse with the four-month regimen and the sixmonth regimen (Gillespie 2014; Jawahar 2013; Jindani 2014). Due to the greater proportion of relapses in the four-month ATT arm, proportions assessed for acquired drug resistance differed between the four-month regimen $(162 / 1392 ; 11.7 \%)$ and the sixmonth regimen (43/873; 4.9\%). Overall, eight people were judged to have developed acquired drug resistance. Two persons in the four-month moxifloxacin-containing ATT regimens in the three trials were detected with acquired drug resistance - one to moxifloxacin and one to isoniazid. The incidence of acquired drug resistance ranged from $0.83 \%(1 / 120)$ in Gillespie 2014 to $7.7 \%$ (1/13) in Jawahar 2013 to 0\% (of 29 assessed) in Jindani 2014. Six people developed acquired drug resistance in the six-month standard ATT arms - three to isoniazid and three to rifampicin. The incidence ranged from 15\% (3/20) in Gillespie 2014 to $13 \%$ (2/15) in Jawahar 2013 to $12.5 \%$ (1/8) in Jindani 2014. Results for the four people with acquired drug resistance in Gillespie 2014 were not unequivocal but were judged probable. We pooled the data for acquired drug resistance from these trials using numbers evaluated for treatment failure in each trial as a more appropriate denominator for assessing acquired drug resistance than only those who experienced treatment failure or relapse. The pooled effect estimate suggests that acquired drug resistance was less frequent with the four-month moxifloxacin-containing ATT regimen than with the standard six-month ATT regimen, but events were rare and 95\% Cls were imprecise (2282 participants, 3 trials; Analysis 1.8).

\section{Adverse events}

\section{Serious adverse events}

All five included studies reported serious adverse events (SAEs) that were fatal or life-threatening, or required hospitalization or a change in treatment regimen. Gillespie 2014 reported that a total of 349 SAEs occurred in 173 participants, with 246 events occurring during the treatment period and 103 during follow-up. Serious adverse events occurred in 62 of $655(9 \%)$ in the isoniazid group and in 52 of $636(8 \%)$ in the ethambutol group, compared with 59 of 639 (9\%) in the control group. The incidence of adverse events, including seizures, clinically significant cardiac toxicity, hypoglycaemia or hyperglycaemia, and peripheral neuropathy, did not significantly differ. Jawahar 2013 noted only two SAEs - a case of jaundice in a person on the six-month regimen and QTC prolongation in a person on the moxifloxacin-ATT regimen. Jindani 2014 reported 12 SAEs among 11 participants on the four-month ATT regimen, four of which were considered possibly or probably related to study medicines. In the control arm, 16 events were reported among 12 participants, with six possibly or probably related to treatment. Velayutham 2014 reported QTc prolongation in five participants in the moxifloxacin group and in one on standard ATT, but all cases were reversible. Other SAEs included hepatitis $(12$ in the moxifloxacin arm and 2 in the control arm) and seizures (four in the moxifloxacin arm and two in the control arm). 
The meta-analysis did not show significant differences between treatment regimens in the incidence of SAEs among 3548 participants in the four trials (Analysis 1.9).

\section{Other adverse events}

In Jawahar 2013, the most common adverse events were gastrointestinal symptoms (nausea, vomiting, abdominal discomfort), which occurred in 25 of 115 (22\%) in the moxifloxacin group and in 15 of 165 (9\%) in the control group. Giddiness or dizziness was also more frequent with moxifloxacin-containing regimens $(17 / 115 ; 15 \%)$ than with standard ATT $(5 / 165 ; 3 \%)$. Arthralgia attributable to pyrazinamide was seen in 3 of $115(3 \%)$ in the four-month regimen and in 4 of $165(2 \%)$ in the six-month regimen.

Velayutham 2014 also reported that arthralgia was significantly greater in the moxifloxacin group ( $25 \%$ of 616 participants) than in the control group (4\% of 164 participants). Skin rash with or without pruritis occurred in $5 \%$ of 616 participants in the moxifloxacin arms and in $4 \%$ of 164 participants in the six-month ATT arm. The other three trials did not report adverse events other than SAEs.

\section{Comparison 2. Gatifloxacin-based four-month ATT regimens versus standard six-month ATT regimens}

Two trials provided data for this intervention. Jawahar 2013 was a three-armed, open-label, equivalence trial, one arm of which randomized 141 adults with drug-sensitive pulmonary tuberculosis to two months of supervised gatifloxacin $400 \mathrm{mg}$ (replacing ethambutol), isoniazid, rifampicin, and pyrazinamide thrice weekly, followed by two months of gatifloxacin, isoniazid, and rifampicin thrice weekly. The 170 participants in the control arm were administered thrice-weekly supervised standard sixmonth ATT. Merle 2014 was an open-label, two-arm, non-inferiority trial that randomized 917 participants to a similar gatifloxacincontaining regimen (also replacing ethambutol) but given daily and compared the effects with 919 participants given a daily, supervised, standard six-month ATT regimen.

We did not find trials that used gatifloxacin to replace isoniazid or to augment standard ATT regimens.

\section{Primary outcome}

\section{Relapse}

Both trials reported on relapse after confirming culture conversion by Löwenstein-Jensen (LJ) solid media to confirm tuberculosis recurrence over 24 months after treatment in people who had become culture negative with treatment. Jawahar 2013 did not differentiate relapse from recurrence but reported that all 19 recurrences in the gatifloxacin-containing ATT arm and 8 of 10 recurrences in the six-month ATT arm occurred within six months after treatment (suggestive of relapse rather than reinfection). In Merle 2014, of 140 participants with culture-positive recurrence, $77(55 \%)$ had strains genotyped by means of a 15locus mycobacterial interspersed repetitive unit-variable-number tandem-repeat analysis. Of these 77 patients, 15 of 20 (75\%) in the gatifloxacin arm and 46 of $57(81 \%)$ in the standard ATT arm had a verified relapse. Relapse was diagnosed in $6.5 \%$ of 155 participants given six months of ATT in Jawahar 2013 and in $7.1 \%$ of 662 people given six months of ATT in Merle 2014. Relapse was more common with the gatifloxacin-containing regimens: $15.6 \%$ of 122 in Jawahar 2013, and 14.6\% of 694 in Merle 2014. Meta-analysis of the two trials showed that relapse was twice as common with the gatifloxacin-containing four-month regimen than with the sixmonth ATT regimen (RR 2.11, $95 \% \mathrm{Cl} 1.56$ to $2.84 ; 1633$ participants, 2 trials; Analysis 2.1).

Jawahar 2013 was at high risk of bias for allocation concealment and excluded HIV-positive individuals. However, meta-analysis results did not reveal any inconsistency in the results. Merle 2014 included HIV-positive participants and undertook subgroup analysis based on HIV status. No significant interaction effects were detected between HIV status and unfavourable outcomes.

As in the previous comparison, we used m-ITT analysis data from both trials for meta-analysis in this review. Sensitivity analyses comparing m-ITT data and per-protocol data showed similar results, as did meta-analysis using all randomized participants (minus late screening failures, treatment failures, and deaths) with imputed relapse rates for missing participants from relapse proportions in the per-protocol analyses reported in the two trials (Table 3; Analysis 2.2; Figure 5). 
Figure 5. Forest plot of comparison: 2 Gatifloxacin-containing 4-month ATT versus standard 6-month ATT regimens, outcome: 2.2 Relapse: sensitivity analysis accounting for missing data.

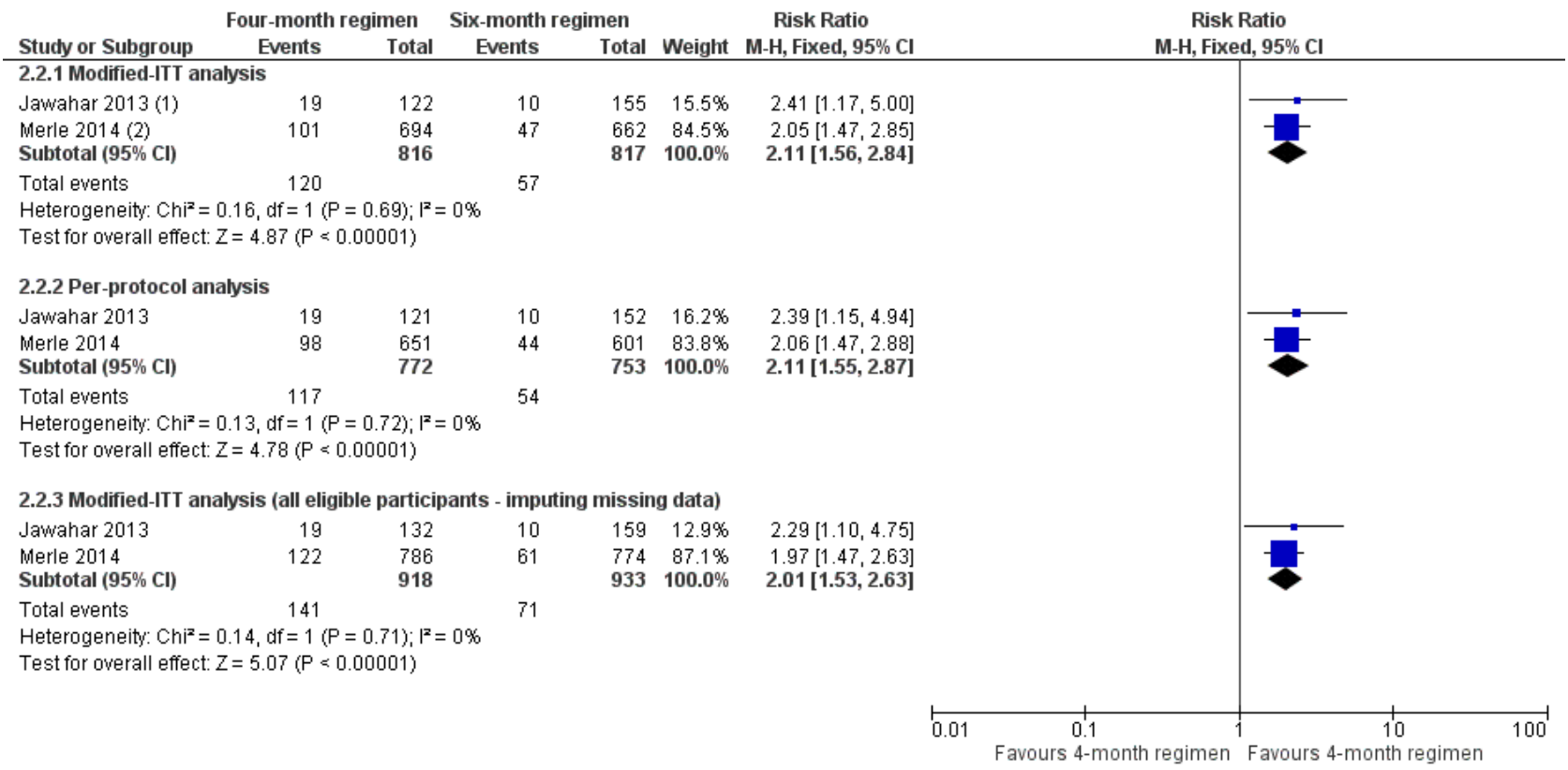

$\underline{\text { Footnotes }}$

(1) Gatifloxacin thrice-weekly for 4 months (replacing ethambutol in intensive phase) versus thrice-weekly standard 6-month regimen; FU: 24 months after treatment

(2) Gatifloxacin daily for 4 months (replacing ethambutol in intensive phase) versus standard daily 6-month regimen; FU: 24 months after treatment

\section{Secondary outcomes}

\section{Death from any cause}

One non-tuberculosis-related death was reported in each of the four-month and six-month arms in Jawahar 2013, In Merle 2014, five deaths in the gatifloxacin arm and nine deaths in the six-month ATT arm occurred during treatment. Two deaths in the gatifloxacin arm and three in the control arm were defined as SAEs. An additional 19 deaths in the gatifloxacin arm and 18 deaths in the standard ATT arm were reported after treatment. Meta-analysis did not reveal significant differences in risk of death due to any cause between ATT regimens (1886 participants, 2 trials; Analysis 2.3).

\section{Treatment discontinuation}

In Jawahar 2013, seven people in each arm discontinued treatment ( $5,2 \%$ of 136 with gatifloxacin-containing ATT, and $4.2 \%$ of 165 with standard ATT); in Merle 2014, 27 of 694 (3.9\%) and 41 of 662 (6.2\%) discontinued treatment. Although the control arm included more people who discontinued treatment in Merle 2014, and the reverse was seen in Jawahar 2013, risk of treatment discontinuation between the two ATT regimens was not appreciably different (1657 participants, 2 trials; Analysis 2.4).

\section{Positive sputum culture at eight weeks}

Gatifloxacin replacing ethambutol in ATT regimens did not offer any advantage over standard ATT in sterilizing sputum at the end of the intensive phase of anti-tuberculosis treatment. At eight weeks $16 \%$ of 1818 participants on the two ATT regimens were sputum positive. Pooled data did not reveal that either intervention was better in sputum conversion at eight weeks (1818 participants, 2 trials; Analysis 2.5).

\section{Treatment failure}

Treatment failure was rare in both trials. In the gatifloxacincontaining ATT arms, 19 of 830 in the two trials (2.1\%) had positive sputum cultures at end of treatment. In the control arms, 21 of 827 (2.5\%) participants experienced treatment failure. Pooled data did not show significant differences in treatment failure between the two ATT regimens (1657 participants, 2 trials; Analysis 2.6).

\section{Acquired drug resistance}

Of the two included trials evaluating gatifloxacin-containing fourmonth ATT regimens versus standard six-month ATT regimens, only Jawahar 2013 reported on acquired drug resistance among 41 participants who experienced culture confirmed treatment failure, or who suffered a recurrence in the six-month ATT arm. Rifampicin resistance developed in one participant and isoniazid resistance in another. None of the participants given the gatifloxacin-containing four-month ATT regimen was detected to have acquired drug resistance. Acquired drug resistance did not differ significantly between the two ATT regimens when the number of participants in each ATT regimen assessed for treatment failure was used as the denominator rather than only the number with treatment failure (301 participants; Analysis 2.7). However, susceptibility to gatifloxacin was not directly evaluated in this trial.

\section{Serious adverse events}

Five people in Jawahar 2013 had SAEs; with gatifloxacin-containing ATT, three had seizures and one had QTc prolongation requiring termination of treatment; and with control ATT, one person had jaundice. In Merle 2014, 20 people in the gatifloxacin arm had 20 SAEs, of which 14 were considered unrelated to treatment; two of three SAEs considered treatment related were deaths. With control ATT, 23 people had 23 SAEs, of which 20 were considered 
unrelated to treatment; two of three considered treatment related were deaths. Pooled effect estimates were similar for both regimens (1993 participants, 2 trials; Analysis 2.8).

\section{Other adverse events}

In Jawahar 2013, nausea, vomiting, and abdominal discomfort $(23 \%)$ and giddiness (18\%) were more frequent among 136 participants given the four-month regimen than among 165 participants on standard ATT ( $9 \%$ and 3\%, respectively). Merle 2014 systematically assessed participants for QTc and blood sugar abnormalities and reported no differences in abnormal peak values of the QTc interval between ATT regimens, nor in episodes of high or low blood sugar, between ATT regimens.

\section{DISCUSSION}

We included five trials that compared fluoroquinolone-containing four-month anti-tuberculosis treatment (ATT) regimens versus standard six-month ATT regimens, recruiting 5825 adults with drug-sensitive pulmonary tuberculosis from 14 countries with high tuberculosis transmission in Asia, Africa, and Latin Ameria. Three were multi-country trials that included a total of 572 HIV-positive people who were not receiving antiretroviral treatment (ART).

\section{Summary of main results}

Moxifloxacin-containing four-month ATT regimens that substitute for ethambutol or isoniazid probably increase relapse following treatment in adults with drug-sensitive pulmonary tuberculosis compared to standard six-month ATT regimens (moderatecertainty evidence; Summary of findings for the main comparison). Compared to standard six-month ATT, four-month ATT regimens that substitute gatifloxacin for ethambutol probably increase relapse following treatment in adults with drug-sensitive tuberculosis (moderate-certainty evidence; Summary of findings 2).

Compared to six-month ATT, four-month ATT regimens containing either moxifloxacin or gatifloxacin probably make little or no difference in treatment failure, death, or serious adverse events (moderate-certainty evidence). Four-month moxifloxacincontaining regimens may not increase the incidence of acquired drug resistance (low-certainty evidence). We are uncertain whether gatifloxacin-containing four-month ATT regimens increase the incidence of acquired drug resistance (very low-certainty evidence). See Summary of findings for the main comparison for moxifloxacincontaining four-month regimens, and Summary of findings 2 for gatifloxacin-containing four-month regimens.

\section{Overall completeness and applicability of evidence}

The trials that met our inclusion criteria evaluated only two of the third-generation fluoroquinolones in use (moxifloxacin and gatifloxacin). Four of the five trials evaluated their effects in replacing ethambutol or isoniazid in shortened ATT regimens. Only one ongoing trial evaluated the effects of adding a fluoroquinolone (moxifloxacin) to standard ATT drugs in shortened regimens, and results for the clinically relevant outcomes of treatment failure and relapse are awaited. Available evidence from the studies in this review indicates that shortened regimens that replace ethambutol or isoniazid with moxifloxacin may not increase acquired drug resistance. We are uncertain whether gatifloxacincontaining regimens will increase acquired drug resistance, as this was assessed in only one trial that used ofloxacin susceptibility as a proxy. Resistance to fluoroquinolones in people with newly diagnosed pulmonary tuberculosis, and in those undergoing retreatment, is increasingly recognized as a problem, particularly in parts of the world where fluoroquinolone use is widespread and is unregulated (Agarwal 2009; Devasia 2009; Selvakumar 2015). Fluoroquinolone-related harms were systematically assessed in all five trials, particularly in the three multi-country trials. Ongoing trials are comparing other four-month regimens versus standard six-month ATT (Characteristics of ongoing studies). Results of these studies will add to the available evidence to inform decisions on whether first-line treatment for drug-sensitive pulmonary tuberculosis can be shortened effectively without compromising safety or increasing relapse or acquired drug resistance.

Two of the three trials of moxifloxacin-containing regimens differentiated relapse from re-infection through genotyping. In the other two trials, most recurrences were considered relapse rather than re-infection, although in a smaller proportion, reinfection may have caused recurrence. ATT treatment primarily affects relapse rates - not re-infection rates; the latter would depend on other factors such as comorbid HIV infection and the intensity of tuberculosis transmission (Wood 2011). The trials in this review were conducted in high tuberculosis-burden countries, where the pressure of tuberculosis transmission is high. These high-burden countries account for $84 \%$ of the burden of tuberculosis worldwide (WHO 2018). Relapse and re-infection are not usually differentiated in tuberculosis control programmes in these countries. It is reassuring to note that in this regard, one of the sensitivity analyses undertaken in Gillespie 2014 and Jindani 2014 included all reinfections under unfavourable outcomes, and effect estimates did not substantially differ from meta-analysis that excluded reinfection.

The trials included in this review excluded children and pregnant or lactating women. They also excluded people with many comorbid conditions such as previous tuberculosis, those with HIV on ART or with low CD4 counts, and those with diabetes. Therefore, results of this review can be applied primarily to adults with drug-sensitive pulmonary tuberculosis without serious comorbid conditions. This review provides evidence of moderate certainty that fourmonth ATT regimens that substitute moxifloxacin or gatifloxacin for isoniazid or ethambutol are probably inferior to standard six-month ATT regimens in preventing relapse (even though there is probably little or no difference in cure). It may be argued that relapse with the four-month regimens would likely not be less in populations with serious comorbid conditions than was reported in the trials in this review.

Nevertheless, extrapolation of the results of this review to people with diabetes (many of whom may have other serious comorbid illnesses) may be more problematic. First, comorbid diabetes mellitus (DM) and tuberculosis are increasingly common, as people with DM have increased risk of developing active tuberculosis; most of this dual burden is found in low- and middle-income, high tuberculosis-burden countries (Al-Rifai 2017; Jeon 2008; Tegegne 2018). People with DM and tuberculosis are more likely to have poorer treatment outcomes than people for whom DM is not comorbid with tuberculosis (Baker 2011). Diabetes increases the risk of treatment failure, death, relapse, and recurrence due to new infection among people with tuberculosis (Baker 2011). Diabetes also increases the odds of developing multi-drug resistant 
tuberculosis (MDR-TB) (Liu 2017; Tegegne 2018). Management of tuberculosis and DM also poses problems due to drug interactions between anti-tuberculosis drugs, particularly rifampicin, and antidiabetic drugs, and adverse drug events are more frequent among those with tuberculosis and DM than in those with tuberculosis alone (Riza 2014).

\section{Certainty of the evidence}

We used the GRADE approach to judge the certainty of evidence for pre-selected outcomes for each comparison in this review (Guyatt 2011a). One of the trials that contributed data to both comparisons in this review was judged at high risk of selection bias due to compromised allocation concealment (Jawahar 2013). However, removal of data from this trial in sensitivity analyses did not alter the direction of effect estimates, so we did not downgrade for risk of bias in the comparison of moxifloxacin-containing four-month versus standard six-month ATT regimens. We downgraded all outcomes by one level for indirectness due to restricted inclusion criteria in all trials, particularly exclusion of people with DM and tuberculosis. People with tuberculosis and DM are four times more likely to relapse than those without DM (Baker 2011). However, they also are more likely to die than people without DM, and this can affect relapse estimates variably. These differences in vulnerability among people with comorbid DM and tuberculosis reduce our certainty in the effect estimates for relapse with shortened versus standard ATT regimens ascertained most often from people without comorbid DM recruited to the trials in this review. Results for this outcome in the comparison of moxifloxacin-containing fourmonth ATT versus standard ATT show inconsistency between large and small effects in favour of the six-month regimen. We graded the certainty of evidence for the primary outcome of relapse in both comparisons as moderate (Summary of findings for the main comparison; Summary of findings 2).

We also graded the certainty of evidence for death due to any cause, treatment discontinuation, and serious adverse events in both comparisons as moderate, downgrading all by one level for indirectness due to restricted inclusion criteria, particularly for those with DM. For these outcomes, the $95 \%$ confidence intervals (Cls) for the risk ratios (RRs) were wide, but events were few and samples size was sufficiently large. The RR and the $95 \% \mathrm{Cl}$ around the RR were precise and indicated little or no difference in clinically appreciable effects with either treatment regimen. Moreover, the primary studies were designed as non-inferiority trials, with the non-inferiority margin set at $6 \%$, and the $95 \%$ $\mathrm{Cls}$ for pooled absolute estimates of risk for the outcomes of death, treatment failure, and serious adverse events in both sets of comparisons were well within this margin. We therefore did not downgrade these outcomes for imprecision. We graded the certainty of evidence for the outcome of acquired drug resistance as low for the comparison of moxifloxacin-based combination regimens, and as very low for the comparison of gatifloxacin-based regimens, because in addition to indirectness, we downgraded these outcomes for imprecision, and additionally for high risk of bias for the comparison with the gatifloxacin-containing regimen, because the sole trial reported baseline imbalance among the proportions with drug resistance (Jawahar 2013).

\section{Potential biases in the review process}

We used standard methods as provided in the Cochrane Handbook for Systematic Reviews of Interventions (Higgins 2011). The literature search covered multiple databases; in addition, we evaluated reference lists of included studies and of relevant systematic reviews for potentially eligible trials. We were unable to formally assess publication bias by using funnel plots because we identified only five relevant trials with outcomes pertinent to this review. We are aware of six ongoing studies that will inform updates of this review. At least two review authors independently screened studies for inclusion, and this was independently verified by a senior review author. Data extraction was done independently by two review authors and was verified independently by two other review authors.

We attempted to account for loss to follow-up for the primary outcome of relapse by using in our main analysis data provided in each trial report's modified intent-to-treat (m-ITT) analysis, because trials included more randomized participants than were included in their per-protocol analyses. The three multi-country trials had shown that the results of sensitivity analyses comparing per-protocol and m-ITT analyses were consistent. However, when data from trials are included in a meta-analysis, pooled estimates can vary depending on how much information is missing for trial outcomes, as well as the magnitude and direction of effect estimates in individual trials. The series of sensitivity analyses that we carried out did not indicate that missing data for relapse influenced the overall results.

We excluded many trials that compared ATT regimens containing fluoroquinolones versus standard ATT regimens (see Characteristics of excluded studies) and reported data for sputum culture positivity at eight weeks - a secondary outcome of this review. This review's inclusion criteria required comparison of shortened tuberculosis regimens versus standard six-month tuberculosis regimens, and because these phase $2 b$ trials were primarily designed to evaluate and report sputum conversion only at two months, they did not fulfil the review's inclusion criteria. On the other hand, we included Velayutham 2014, which reported sputum culture results at two months but did not provide data on treatment success or relapse. However, unlike the phase $2 b$ trials that we excluded, this study was designed as a phase 3 trial that fulfilled this review's selection criteria. Sputum conversion data at eight weeks was a pre-stated secondary outcome, and the interim report included adverse events during treatment, further justifying its inclusion. We also excluded data for sputum conversion from one of the trial arms in Jindani 2014, which used moxifloxacin in place of isoniazid but for six months. Data for sputum culture conversion at two months from the four-month and six-month moxifloxacin arms were combined and reported, and we could not use these data. However, we will review in the following section the data for sputum culture conversion from these trials for other published work to take into account the totality of trial evidence for this outcome.

\section{Agreements and disagreements with other studies or reviews}

An earlier Cochrane Review on fluoroquinolones for treating pulmonary tuberculosis found only ongoing trials of fluoroquinolone-containing shortened regimens compared to sixmonth standard ATT regimens that have now been included in this present review (Ziganshina 2013). Ziganshina 2013 included the phase $2 b$ trials that were excluded from the present review. Other systematic reviews on fluoroquinolones for treating people with drug-sensitive pulmonary tuberculosis also included these phase 
2b trials (Lee 2016; Li 2016; Ruan 2016), as well as the phase 3 trials included in this review. Meta-analyses of data for sputum positivity at eight weeks from the phase $2 b$ and phase 3 trials (including combined data from the moxifloxacin-containing fourand six-month arms in Jindani 2014) included in these reviews showed similar results as this review for sputum conversion at eight weeks. These systematic reviews also reported similar results for the other outcomes reported in this review.

The effect of shortened treatment regimens for people with noncavitary disease was not an objective of this review. However, another systematic review sought to evaluate whether people with non-cavitary tuberculosis may have better outcomes than those with cavitary disease with shorter regimens, because the bacterial load is less with non-cavitary pulmonary tuberculosis. Only the three multi-country trials included in this review met their inclusion criteria and provided data for participants with non-cavitary disease (Gillespie 2014; Jindani 2014; Merle 2014). They used data in meta-analysis from 1066 participants from the three trials who had non-cavitary pulmonary tuberculosis. They had intended to study the effects of fluoroquinolone-containing regimens on relapse and cure but could not find disaggregated data for these outcomes for people with non-cavitary tuberculosis in the three trials. They used the composite 'unfavourable outcome' in these trials and used a margin of $6 \%$ in the risk ratio (RR) for pooled estimates to indicate non-inferiority. The 95\% confidence interval $(\mathrm{CI})$ for the pooled RR for unfavourable outcomes using data from the three trials exceeded this margin, and the results were heterogeneous. In subgroup analyses of pooled data from trials using daily treatment (Gillespie 2014; Merle 2014), the results were homogeneous and the $95 \% \mathrm{Cl}$ for the RR was within the noninferiority margin (RR $1 \%, 95 \% \mathrm{Cl}-3 \%$ to $5 \%$; 965 participants, 2 trials). Also, in subgroup analysis using pooled data from the arms of these two trials when fluoroquinolones were substituted for ethambutol and were compared to six-month ATT, the $95 \% \mathrm{Cl}$ for the pooled RR for an unfavourable outcome was within the noninferiority margin (RR $-1 \%, 95 \% \mathrm{Cl}-5 \%$ to $4 \% ; 857$ participants, 2 trials). Pooling data from the three trials for serious adverse events among participants with non-cavitary disease also showed no difference for the flouroquinolone-containing regimens versus the six-month regimens, with the $95 \% \mathrm{Cl}$ for the RR clearly within the non-inferiority margin (RR $0 \%, 95 \% \mathrm{Cl}-2 \%$ to $1 \%$; 4811 participants, 3 trials). Alipanah 2016 concluded that fourmonth daily regimens substituting ethambutol with gatifloxacin or moxifloxacin may be non-inferior to standard therapy for patients with culture confirmed, non-cavitary, drug-susceptible pulmonary tuberculosis. These review authors acknowledged that these estimates may be prone to error because they had to use data from a mix of per-protocol and intention-to-treat analysis data from the trials in their analysis.

The suggestion from the results in Alipanah 2016 that increased relapse proportions seen with moxifloxacin- and gatifloxacincontaining regimens compared to the standard six-month regimen in the present review may be due to inclusion of people with cavitary lung disease due to tuberculosis needs verification. Support for this observation comes from a pooled analysis of individual patient data-sets of 3411 participants from Gillespie 2014, Jindani 2014, and Merle 2014 (Imperial 2018). This analysis identified two subgroups of participants that differed in their response to the four-month regimens. A subgroup of patients with drug-susceptible tuberculosis with low grades of sputum positivity or absence of cavitation at baseline assessments was at lower risk for unfavourable outcomes, and this population (with either of these low-risk characteristics) constituted $47 \%$ of the 3405 participants in the three trials. The four-month fluoroquinolone regimens in these trials were effective in reducing the risk of unfavourable outcomes in this population with "minimal disease". Another subgroup of participants with a smear grade of $3+$ and the presence of cavitation on chest radiographs at baseline $34 \%$ of total sample) had unfavourable outcomes. Data from this pooled analysis suggest that this "hard-to-treat" population may require treatment for longer than those given the standard sixmonth regimen to achieve optimal outcomes (Imperial 2018). These observations from Alipanah 2016 and Imperial 2018 have implications of heuristic value for the design and interpretation of future trials on this topic.

\section{AUTHORS' CONCLUSIONS}

\section{Implications for practice}

Evidence to date does not support the use of fluoroquinolonecontaining shortened ATT regimens for adults with newly diagnosed drug-sensitive pulmonary tuberculosis. Although there is probably little or no difference in cure or serious adverse events with four-month ATT regimens that replace ethambutol with moxifloxacin or gatifloxacin, or isoniazid with moxifloxacin, compared to standard six-month ATT regimens, the shortened regimens will probably increase relapse substantially.

\section{Implications for research}

Six ongoing trials will provide more evidence on shortened ATT regimens compared to standard six-months ATT regimens. One is CTRI/2008/091/000024, which reported interim results for sputum culture conversion at eight weeks with moxifloxacin added to standard ATT drugs (Velayutham 2014). Moxifloxacin added to standard ATT resulted in significantly fewer people with positive sputum culture at eight weeks than were seen with standard ATT in a direct comparison and also compared to regimens in which moxifloxacin was substituted for ethambutol or isoniazid, in indirect comparisons in this review (Analysis 1.6). This trial anticipates recruiting 1650 participants and, when results are available, will provide data on treatment failure and on relapse assessed over 24 months after treatment completion to evaluate whether this early advantage with moxifloxacin addition translates into cure and relapse that are not inferior to those seen with standard six-month treatment. Four moxifloxacin-containing arms are comparing moxifloxacin added to isoniazid, rifampicin, ethambutol, and pyrazinamide given daily for three months, or given daily for four months, versus pyrazinamide and ethambutol given only for the first two months; or given daily for two months and thrice weekly for two months versus pyrazinamide and ethambutol only in the first two months; or given daily for two months and thrice weekly for two months with ethambutol continued and pyrazinamide omitted. Interim results in 2011 after 605 participants were enrolled showed that recurrence rates were similar in the four-month moxifloxacin-containing arms but tuberculosis recurrence was significantly higher among those treated with the three-month moxifloxacin regimen compared to the other regimens. The data safety monitoring board halted recruitment to the three-month moxifloxacin regimen. 
Four other ongoing trials are recruiting adults with drug-sensitive pulmonary tuberculosis into shortened regimens compared to standard six-month ATT regimens. The RIFASHORT trial will compare rifampicin given at higher doses (1200 mg daily or 1800 mg daily) added to standard ATT (NCT02581527). NCT02410772 has two intervention arms, one of which is comparing daily rifapentine instead of rifampicin added to standard ATT drugs for eight weeks, followed by rifapentine and isoniazid for nine weeks. Another arm adds moxifloxacin to the above regimen. NCT02901288 also has two intervention arms. One is evaluating 4.5 months of isoniazid, rifampin, pyrazinamide, ethambutol, and levofloxacin, and the other is comparing 4.5 months of isoniazid, rifampin, pyrazinamide, and ethambutol versus standard ATT. In the STAND trial (NCT02342886), two of the intervention arms will evaluate pretomanid (PA 824) $200 \mathrm{mg}$ or $100 \mathrm{mg}$ daily added to moxifloxacin and pyrazinamide for 17 weeks compared to standard six-month ATT.

One ongoing trial is evaluating two months of standard ATT followed by rifampicin and isoniazid, with or without ethambutol, versus standard six-month ATT in children 0 to 16 years (ISRCTN63579542). We did not find any other ongoing trials in children.

These ongoing trials hope to recruit a total of 10,250 participants, and when they are published will provide additional data for the outcomes in this review and for additional comparisons. If data are separately available in these trials for the outcomes in this review, particularly for relapse, treatment failure, and adverse events among those with and without cavitary lung disease and according to baseline sputum smear grading, this will enable evaluation of the observations in Alipanah 2016 and Imperial 2018 that those without cavitary lung disease and low smear grade may form a subgroup of people with drug-sensitive pulmonary tuberculosis for whom shortened ATT regimens might prove most effective in terms of cure and without increase in relapse compared to standard six-month ATT.

\section{ACKNOWLEDGEMENTS}

The Academic Editor is Professor Mical Paul.

We thank Vittoria Lutje, the Information Specialist for the Cochrane Infectious Diseases Group (CIDG), for her guidance with the literature search strategy. We are grateful to Paul Garner, CIDG Coordinating Editor, for his comments and support. We also gratefully acknowledge helpful comments from an anonymous peer reviewer and from Dr Marylou Murray. We thank the Contact Editor for this review, Mical Paul, and the CIDG statistician, Marty Richardson, for their painstaking peer review and helpful comments. We thank $\mathrm{Dr}$ MS Jawahar, lead author of an included trial, for providing further information about methods and recruitment-related data.

The editorial base of the Cochrane Infectious Diseases Group is funded by UK aid from the UK government for the benefit of lowand middle-income countries (project number 300342-104). The views expressed do not necessarily reflect the UK government's official policies.

The review was supported by READ-It (project number 300342-104). RK and PT was supported by the grant previous to READ-It (Grant: 5242).

This review was undertaken as part of the training course seeking to build capacity on undertaking systematic reviews in India, especially in the context of tuberculosis. The training project was conceived and implemented by the South East Asia office of the International Union Against Tuberculosis and Lung Diseases (The Union) in collaboration with the Central TB Division, Ministry of Health and Family Welfare, Government of India, and Cochrane South Asia. The training was generously supported by the US Agency for International Development (USAID). We also thank the Department for International Development (DFID), UK, for funding the Global Operational Research Fellowship Programme at The Union, Paris, France, in which JT and SS work as Senior Operational Research Fellows. 
R E F E R E N C E S

\section{References to studies included in this review}

Gillespie 2014 \{published data only\}

* Gillespie SH, Crook AM, McHugh TD, Mendel CM, Meredith SK, Murray SR, et al. Four-month moxifloxacin-based regimens for drug-sensitive tuberculosis. New England Journal of Medicine 2014;371(17):1577-87. [DOI: 10.1056/NEJMoa1407426; NCT00864383]

NCT00864383. Controlled comparison of two moxifloxacin containing treatment shortening regimens in pulmonary tuberculosis (REMoxTB). https://clinicaltrials.gov/ct2/show/ NCT00864383 (accessed 1 September 2018).

Phillips PJP, Mendel CM, Burger D A, Crook AM, Nunn AJ, Dawson $\mathrm{R}$, et al. Limited role of culture conversion for decision-making in individual patient care and for advancing novel regimens to confirmatory clinical trials. BMC Medicine 2016;14:19. [DOI: 10.1186/s12916-016-0565-y]

Phillips PPJ, Mendel CM, Nunn AJ, McHugh TD, Crook AM, Hunt $R$, et al. A comparison of liquid and solid culture for determining relapse and durable cure in phase III TB trials for new regimens. BMC Medicine 2017;15(1):207. [DOI: 10.1186/ s12916-017-0955-9]

\section{Jawahar 2013 \{published and unpublished data\}}

CTRI/2012/10/003060. A study of the efficacy and tolerability of moxifloxacin and gatifloxacin containing regimens in the treatment of patients with sputum-positive pulmonary tuberculosis. http://ctri.nic.in/Clinicaltrials/showallp.php? mid1=5124\&EncHid=\&userName=CTRI/2012/10/003060 (accessed 28 August 2018). [CTRI/2012/10/003060]

* Jawahar MS, Banurekha VV, Paramasivan CN, Rahman F, Ramachandran R, Venkatesan P, et al. Randomized clinical trial of thrice-weekly 4-month moxifloxacin or gatifloxacin containing regimens in the treatment of new sputum positive pulmonary tuberculosis patients. PLOS One 2013;8(7):e67030. [PUBMED: 23843980]

\section{Jindani 2014 \{published data only (unpublished sought but not} used)\}

ISRCTN44153044. An international multicentre controlled clinical trial to evaluate high dose RIFApentine and a QUINolone in the treatment of pulmonary tuberculosis. https:// doi.org/10.1186/ISRCTN44153044 (accessed 26 August 2018). [ISRCTN44153044]

* Jindani A, Harrison TS, Nunn AJ, Phillips PPJ, Churchyard GJ, Charalambous $\mathrm{S}$, et al. High-dose rifapentine with moxifloxacin for pulmonary tuberculosis. New England Journal of Medicine 2014;371(17):1599-608.

\section{Merle 2014 \{published data only\}}

* Merle CS, Fielding K, Sow OB, Gninafon M, Lo MB, Mthiyane T, et al. A four-month gatifloxacin-containing regimen for treating tuberculosis. New England Journal of Medicine 2014;371(17):1588-98.
Merle CS, Fielding K, Sow OB, Gninafon M, Lo MB, Mthiyane T, et al. Correction: $A$ four-month gatifloxacin-containing regimen for treating tuberculosis. New England Journal of Medicine 2015;372(17):1677. [PUBMED: 25901444]

Merle CS, Sismanidis C, Sow OB, Gninafon M, Horton J, Lapujade O, et al. A pivotal registration phase III, multicenter, randomized tuberculosis controlled trial: design issues and lessons learnt from the Gatifloxacin for TB (OFLOTUB) project. Trials 2012;13:61. [DOI: 10.1186/1745-6215-13-61]

NCT00216385. A controlled trial of a 4-month quinolonecontaining regimen for the treatment of pulmonary tuberculosis. https://clinicaltrials.gov/ct2/show/NCT00216385 (accessed 30 June 2018). [NCT00216385]

Olliaro PL, Merle C, Mthiyane T, Bah B, Kassa F, Amukoye E, et al. Effects on the QT interval of a gatifloxacin-containing regimen versus standard treatment of pulmonary tuberculosis. Antimicrobial Agents and Chemotherapy 2017;61(7):pii: e01834-16. [DOI: 10.1128/AAC.01834-16]

Velayutham 2014 \{published and unpublished data\} CTRI/2008/091/000024. Randomised clinical trial to study the efficacy and tolerability of 3- and 4-month regimens containing moxifloxacin in the treatment of patients with sputum smear and culture positive pulmonary tuberculosis. http://ctri.nic.in/Clinicaltrials/showallp.php? mid1=68\&EncHid=\&userName=CTRI/2008/091/000024 (accessed 28 August 2018). [CTRI/2008/091/000024]

* Velayutham BV, Allaudeen IS, Sivaramakrishnan GN, Perumal V, Nair D, Chinnaiyan P, et al. Sputum culture conversion with moxifloxacin-containing regimens in the treatment of patients with newly diagnosed sputum-positive pulmonary tuberculosis in South India. Clinical Infectious Diseases 2014;59(10):e142-9. [CTRI 2008/091/000024; PUBMED: 25028463]

\section{References to studies excluded from this review}

Alavi 2009 \{published data only\}

Alavi SM. Comparative evaluation of three and six month therapeutic regimens for smear negative pulmonary tuberculosis. Jundishapur Journal of Microbiology 2009;2(1):14-7.

\section{Burman 2006 \{published data only\}}

Burman WJ, Goldberg S, Johnson JL, Muzanye G, Engle M, Mosher AW, et al. Moxifloxacin versus ethambutol in the first 2 months of treatment for pulmonary tuberculosis. American Journal of Respiratory and Critical Care Medicine 2006;174(3):331-8. [DOI: 10.1164/rccm.200603-3600C]

\section{Conde 2009 \{published data only\}}

Conde MB, Efron A, Loredo C, De Souza GR, Graça NP, Cezar MC, et al. Moxifloxacin versus ethambutol in the initial treatment of tuberculosis: a double-blind, randomised, controlled phase II trial. Lancet 2009;373(9670):1183-9. 
Conde 2016 \{published data only\}

Conde MB, Mello FC, Duarte RS, Cavalcante SC, Rolla V, et al. A phase 2 randomized trial of a rifapentine plus moxifloxacinbased regimen for treatment of pulmonary tuberculosis. PLOS One 2016;11(5):e0154778. [DOI: 10.1371/journal.pone.0154778]

\section{Dorman 2009 \{published data only\}}

Dorman SE, Johnson JL, Goldberg S, Muzanye G, Padayatchi N, Bozeman L, et al. Substitution of moxifloxacin for isoniazid during intensive phase treatment of pulmonary tuberculosis. American Journal of Respiratory and Critical Care Medicine 2009;180(3):273-80. [DOI: 10.1164/rccm.200901-00780C]

\section{El-Sadr 1998 \{published data only\}}

El-Sadr WM, Perlman DC, Matts JP, Nelson ET, Cohn DL, Salomon N, et al. Evaluation of an intensive intermittentinduction regimen and duration of short-course treatment for human immunodeficiency virus-related pulmonary tuberculosis. Terry Beirn Community Programs for Clinical Research on AIDS (CPCRA) and the AIDS Clinical Trials Group (ACTG). Clinical Infectious Diseases 1998;26(5):1148-58.

\section{Johnson 2009 \{published data only\}}

Johnson JL, Hadad DJ, Dietze R, Maciel EL, Sewali B, Gitta P, et al. Shortening treatment in adults with noncavitary tuberculosis and 2-month culture conversion. American Journal of Respiratory and Critical Care Medicine 2009;180(6):558-63. [PUBMED: 19542476]

\section{Kohno 1992 \{published data only\}}

Kohno S, Koga H, Kaku M, Maesaki S, Hara K. Prospective comparative study of ofloxacin or ethambutol for the treatment of pulmonary tuberculosis. Chest 1992;102(6):1815-8. [PUBMED: 1446494]

\section{Rustomjee 2008 \{published data only\}}

Rustomjee R, Lienhardt C, Kanyok T, Davies GR, Levin J, Mthiyane T, et al. A phase II study of the sterilising activities of ofloxacin, gatifloxacin and moxifloxacin in pulmonary tuberculosis. International Journal of Tuberculosis and Lung Disease 2008;12(2):128-38. [PUBMED: 18230244]

\section{Tuberculosis Research Centre 1986 \{published data only\}}

Tuberculosis Research Centre, Madras, and National Tuberculosis Institute, Bangalore. A controlled clinical trial of 3- and 5-month regimens in the treatment of sputumpositive pulmonary tuberculosis in south India. American Review of Respiratory Disease 1986;134(1):27-33. [DOI: 10.1164/ arrd.1986.134.1.27]

\section{Tuberculosis Research Centre 2002 \{published data only\}}

Jawahar MS, Rahman F, Narayanan, et al. Shortening short course chemotherapy: a randomised clinical trial for treatment of smear positive pulmonary tuberculosis with regimens using ofloxacin in the intensive phase. Indian Journal of Tuberculosis 2002;49(1):27.

\section{References to ongoing studies}

CTRI/2008/091/000024 \{published data only\}

CTRI/2008/091/000024. Randomised clinical trial to study the efficacy and tolerability of 3- and 4-month regimens containing moxifloxacin in the treatment of patients with sputum smear and culture positive pulmonary tuberculosis. http://ctri.nic.in/Clinicaltrials/showallp.php? mid1=68\&EncHid=\&userName=CTRI/2008/091/000024 (accessed 28 August 2018). [CTRI/2008/091/000024]

ISRCTN63579542 \{published data only\}

Shorter treatment for minimal TB in children (SHINE) Protocol Version 2.1. www.ctu.mrc.ac.uk/research/documents/ tb_protocols/shine_protocol2.12015 (accessed 4 September 2018). [ ISRCTN63579542]

ISRCTN63579542. SHINE study. Shorter treatment for minimal TB in children. www.isrctn.com/ISRCTN63579542 (accessed 17 July 2018).

NCT02342886 \{published data only\}

NCT02342886. Shortening treatment by advancing novel drugs (STAND). https://clinicaltrials.gov/ct2/show/record/ NCT02342886?term=pa-824+moxifloxacin (accessed 28 July 2018). [NCT02342886]

NCT02410772 \{published data only\}

NCT02410772. Rifapentine-containing tuberculosis treatment shortening regimens. https://clinicaltrials.gov/show/ NCT02410772 (accessed 2 March 2018). [NCT02410772]

\section{NCT02581527 \{published data only\}}

NCT02581527. An international multicentre controlled clinical trial to evaluate $1200 \mathrm{mg}$ and $1800 \mathrm{mg}$ rifampicin daily in the reduction of treatment duration for pulmonary tuberculosis from 6 months to 4 months (RIFASHORT). https:// clinicaltrials.gov/show/NCT02581527 (accessed 10 March 2018). [NCT02581527]

\section{NCT02901288 \{published data only\}}

Gao M, Gao J, Du J, Liu Y, Zhang Y, Ma L, et al. Efficacy of ultrashort course chemotherapy for new smear positive drug susceptible pulmonary tuberculosis: study protocol of a multicenter randomized controlled clinical trial. BMC Infectious Diseases 2017;17:435. [DOI: 10.1186/s12879-017-2505-7; NCT02901288]

NCT02901288. Shortened regimens for drug-susceptible pulmonary tuberculosis. https://clinicaltrials.gov/ct2/show/ NCT02901288 (accessed 10 July 2018). [NCT02901288]

\section{Additional references}

\section{Abraha 2015}

Abraha I, Cherubini A, Cozzolino F, De Florio R, Luchetta ML, Rimland JM, et al. Deviation from intention to treat analysis in randomised trials and treatment effect estimates: metaepidemiological study. BMJ 2015;350:h2445. [DOI: 10.1136/ bmj.h2445] 


\section{Agarwal 2009}

Agrawal D, Udwadia ZF, Rodriguez C, Mehta A. Increasing incidence of fluoroquinolone resistant Mycobacterium tuberculosis in Mumbai, India. International Journal of Tuberculosis and Lung Disease 2009;13(1):79-83.

\section{Al-Rifai 2017}

Al-Rifai RH, Pearson F, Critchley JA, Abu-Raddad LJ. Association between diabetes mellitus and active tuberculosis: a systematic review and meta-analysis. PLOS One 2017;12(11):e0187967. [10.1371/journal.pone.0187967]

\section{Alipanah 2016}

Alipanah N, Cattamanchi A, Menzies R, Hopewell PC, Chaisson RE, Nahid P. Treatment of non-cavitary pulmonary tuberculosis with shortened fluoroquinolone-based regimens: a meta-analysis. International Journal of Tuberculosis and Lung Disease 2016;20(11):1522-8. [DOI: 10.5588/ijtld.16.0217]

\section{Andries 2005}

Andries K, Verhasselt P, Guillemont J, Göhlmann HW, Neefs JM, Winkler $\mathrm{H}$, et al. A diarylquinoline drug active on the ATP synthase of Mycobacterium tuberculosis. Science 2005;307(5707):223-7.

\section{Baker 2011}

Baker MA, Harries AD, Jeon CY, Hart JE, Kapur A, Lönnroth K, et al. The impact of diabetes on tuberculosis treatment outcomes: a systematic review. BMC Medicine 2011;9:18. [DOI: 10.1186/1741-7015-9-81]

\section{Balshem 2011}

Balshem H, Helfand M, Schunemann HJ, Oxman AD, Kunz R, Brozek J, et al. GRADE guidelines. 3. Rating the quality of evidence. Journal of Clinical Epidemiology 2011;64(4):401-6.

\section{Boeree 2017}

Boeree MJ, Heinrich N, Aarnoutse R, Diacon AH, Dawson R, Rehal S, et al. High-dose rifampicin, moxifloxacin, and SQ109 for treating tuberculosis: a multi-arm, multi-stage randomised controlled trial. Lancet Infectious Diseases 2017;17(1):39-49. [DOI: 10.1016/S1473-3099(16)30274-2]

\section{Conde 2011}

Conde MB, Lapa E Silva JR. New regimens for reducing the duration of the treatment of drug-susceptible pulmonary tuberculosis. Drug Development Research 2011;72(6):501-8. [DOI: 10.1002/ddr.20456]

\section{D'Ambrosio 2015}

D'Ambrosio L, Centis R, Sotgiu G, Pontali E, Spanevello A, Migliori GB. New anti-tuberculosis drugs and regimens: 2015 update. ERJ Open Research 2015;1(1):00010-2015.

\section{DAIDS 2009}

U.S. Department of Health and Human Services, National Institutes of Health, National Institute of Allergy and Infectious Diseases, Division of AIDS (DAIDS). Division of AIDS Table for Grading the Severity of Adult and Pediatric Adverse Events, Version 1.0. [Updated August 2009].. Available from: https:// rsc.niaid.nih.gov/sites/default/files/table-for-grading-severity- of-adult-pediatric-adverse-events.pdf (accessed 21 September 2018)

\section{Dawson 2015}

Dawson R, Diacon AH, Everitt D, van Niekerk C, Donald PR, Burger DA, et al. Efficiency and safety of the combination of moxifloxacin, pretomanid (PA-824), and pyrazinamide during the first 8 weeks of antituberculosis treatment: a phase $2 b$, open-label, partly randomised trial in patients with drugsusceptible or drug-resistant pulmonary tuberculosis. Lancet 2015;385(9979):1738-47.

\section{Deeks 2011}

Deeks JJ, Higgins JPT, Altman DG (editors). Chapter 9 Analysing data and undertaking meta-analyses. In: Higgins JP, Green S, editor(s). Cochrane Handbook for Systematic Reviews of Interventions Version 5.1.0 (updated March 2011). The Cochrane Collaboration, 2011. Available from handbook.cochrane.org. The Cochrane Collaboration, 2011. Available from www.cochrane-handbook.org.

\section{Devasia 2009}

Devasia RA, Blackman A, Gebretsadik T, Griffin M, Shintani A, May C, et al. Fluoroquinolone resistance in Mycobacterium tuberculosis: the effect of duration and timing of fluoroquinolone exposure. American Journal of Respiratory and Critical Care Medicine 2009;180(4):365-70. [DOI: 10.1164/ rccm.200901-01460C]

\section{Diacon 2012}

Diacon AH, Donald PR, Pym A, Grobusch M, Patientia RF, Mahanyele R, et al. Randomized pilot trial of eight weeks of bedaquiline (TMC207) treatment for multidrug-resistant tuberculosis: long-term outcome, tolerability, and effect on emergence of drug resistance. Antimicrobial Agents and Chemotherapy 2012;56(6):3271-6.

\section{Diacon 2014}

Diacon AH, Pym A, Grobusch MP, de los Rios JM, Gotuzzo E, Vasilyeva I, et al. Multidrug-resistant tuberculosis and culture conversion with bedaquiline. New England Journal of Medicine 2014;371(8):723-32.

\section{Fergusson 2002}

Fergusson D, Aaron SD, Guyatt G, Hebert, P. Post-randomization exclusions: the intention to treat principle and excluding patients from analysis. BMJ 2002;325:652-4.

\section{Gelband 1999}

Gelband H. Regimens of less than six months for treating tuberculosis. Cochrane Database of Systematic Reviews 1999, Issue 4. [DOI: 10.1002/14651858.CD001362]

\section{Ginsberg 2010}

Ginsberg AM. Tuberculosis drug development: progress, challenges, and the road ahead. Tuberculosis (Edinburgh, Scotland) 2010;90(3):162-7. 


\section{Ginsburg 2003}

Ginsburg AS, Grosset JH, Bishai WR. Fluoroquinolones, tuberculosis, and resistance. Lancet. Infectious Diseases 2003;3(7):432-42.

\section{Gler 2012}

Gler MT, Skripconoka V, Sanchez-Garavito E, Xiao H, CabreraRivero JL, Vargas-Vasquez DE, et al. Delamanid for multidrugresistant pulmonary tuberculosis. New England Journal of Medicine 2012;366(23):2151-60.

\section{GRADEpro GDT [Computer program]}

McMaster University (developed by Evidence Prime). GRADEpro GDT. Version accessed 12 November 2017. Hamilton (ON): McMaster University (developed by Evidence Prime), 2015.

\section{Guyatt 2011a}

Guyatt G, Oxman AD, Akl EA, Kunz R, Vist G, Brozek J, et al. GRADE guidelines. 1. Introduction - GRADE evidence profiles and summary of findings tables. Journal of Clinical Epidemiology 2011;64(4):383-94. [PUBMED: 21195583]

\section{Guyatt 2011b}

Guyatt GH1, Oxman AD, Kunz R, Brozek J, Alonso-Coello P, Rind $D$, et al. GRADE guidelines. 6 . Rating the quality of evidence - imprecision. Journal of Clinical Epidemiology 2011;64(12):1283-93. [PUBMED: 21839614]

\section{Guyatt 2011c}

Guyatt GH, Oxman AD, Kunz R, Woodcock J, Brozek J, Helfand $M$, et al. GRADE guidelines. 7. Rating the quality of evidence - inconsistency. Journal of Clinical Epidemiology 2011;64(12):1294-302. [PUBMED: 21803546]

\section{Haverkamp 2012}

Haverkamp W, Kruesmann F, Fritsch A, van Veenhuyzen D, Arvis P. Update on the cardiac safety of moxifloxacin. Current Drug Safety 2012;7(2):149-63. [PUBMED: 22873499]

\section{Higgins 2011}

Higgins JPT, Green S (editors). Cochrane Handbook for Systematic Reviews of Interventions Version 5.1.0 (updated March 2011). Available from www. handbook.cochrane.org. The Cochrane Collaboration, 2011.

\section{Hultcrantz 2017}

Hultcrantz M, Rind D, Akl EA, Treweek S, Mustafa RA, lorio A, et al. The GRADE Working Group clarifies the construct of certainty of evidence. Journal of Clinical Epidemiology 2017;87:4-13.

\section{Imperial 2018}

Imperial MZ, Nahid P, Phillips PPJ, Davies GR, Fielding K, Hanna D, et al. A patient-level pooled analysis of treatmentshortening regimens for drug-susceptible pulmonary tuberculosis. Nature Medicine 2018;24(11):1708-15. [DOI: 10.1038/s41591-018-0224-2]

\section{Jeon 2008}

Jeon CY, Murray MB. Diabetes mellitus increases the risk of active tuberculosis: a systematic review of 13 observational studies. PLOS Medicine 2008;5:e152. [PUBMED: 10.1371/

journal.pmed.0050152]

\section{Kerantzas 2017}

Kerantzas CA, Jacobs WR. Origins of combination therapy for tuberculosis: lessons for future antimicrobial development and application. mBio 2017;8(2):e01586-16.

\section{Lee 2016}

Lee HW, Lee JK, Kim E, Yim J-J, Lee C-H. The effectiveness and safety of fluoroquinolone-containing regimen as a first-line treatment for drug-sensitive pulmonary tuberculosis: a systematic review and meta analysis. PLOS One 2015;11(7):e0159827. [10.1371/ journal.pone.015982]

\section{Lessem 2015}

Lessem E, Cox H, Daniels C, Furin J, McKenna L, Mitnick CD, et al. Access to new medications for the treatment of drugresistant tuberculosis: patient, provider and community perspectives. International Journal of Infectious Diseases 2015;32:56-60.

\section{Li 2016}

Li D, Wang T, Shen S, Cheng S, Yu J, Zhang Y, et al. Effects of flouroquinolones in newly diagnosed, sputum-positive tuberculosis therapy: a systematic review and network meta analysis. PLOS One 2015;10(12):e0145066. [10.1371/ journal.pone.0145066]

\section{Lienhardt 2010}

Lienhardt C, Vernon A, Raviglione MC. New drugs and new regimens for the treatment of tuberculosis: review of the drug development pipeline and implications for national programmes. Current Opinion in Pulmonary Medicine 2010;16(3):186-93.

\section{Liu 2017}

Liu Q, Li W, Xue M, Chen Y, Du X, Wang C, et al. Diabetes mellitus and the risk of multidrug resistant tuberculosis: a metaanalysis. Scientific Reports 2017;7(1):1090. [DOI: 10.1038/ s41598-017-01213-5]

\section{Ma 2010}

Ma Z, Lienhardt C, Mcllleron H, Nunn AJ, Wang X. Global tuberculosis drug development pipeline: the need and the reality. Lancet 2010;375(9731):2100-9.

\section{Matsumoto 2006}

Matsumoto M, Hashizume H, Tomishige T, Kawasaki M, Tsubouchi H, Sasaki H, et al. OPC-67683, a nitro-dihydroimidazooxazole derivative with promising action against tuberculosis in vitro and in mice. PLOS Medicine 2006;3(11):e466.

\section{Mitchison 1985}

Mitchison DA. The action of antituberculosis drugs in shortcourse chemotherapy. Tubercle 1985;66(3):219-25. 


\section{Moadebi 2007}

Moadebi S, Harder CK, Fitzgerald MJ, Elwood KR, Marra F. Fluoroquinolones for the treatment of pulmonary tuberculosis. Drugs 2007;67(14):2077-99.

\section{Munsiff 2006}

Munsiff SS, Kambili C, Ahuja SD. Rifapentine for the treatment of pulmonary tuberculosis. Clinical Infectious Diseases 2006;43(11):1468-75.

\section{Review Manager 2014 [Computer program]}

Nordic Cochrane Centre, The Cochrane Collaboration. Review Manager 5 (RevMan 5). Version 5.3. Copenhagen: Nordic Cochrane Centre, The Cochrane Collaboration, 2014.

\section{Rifat 2018}

Rifat D, Prideaux B, Savic RM, Urbanowski ME, Parsons TL, Luna B, et al. Pharmacokinetics of rifapentine and rifampin in a rabbit model of tuberculosis and correlation with clinical trial data. Science Translational Medicine 2018;10(435):pii: eaai7786. [DOI: 10.1126/scitranslmed.aai7786]

\section{Riza 2014}

Riza AL, Pearson F, Ugarte-Gil C, Alisjahbana B, van de Vijver S, Panduru NM, et al. Clinical management of concurrent diabetes and tuberculosis and the implications for patient services. Lancet Diabetes and Endocrinology 2014;2(9):740-53. [PUBMED: 25194887]

\section{Ruan 2016}

Ruan Q, Liu Q, Sun F, Shao L, Jin J, Yu S, et al. Moxifloxacin and gatifloxacin for initial therapy of tuberculosis: a meta-analysis of randomized clinical trials. Emerging Microbes and Infections 2016;5:e12. [DOI: 10.1038/emi.2016.12]

\section{Rubinstein 2002}

Rubinstein E, Camm J. Cardiotoxicity of fluoroquinolones. Journal of Antimicrobial Chemotherapy 2002;49(4):593-6.

\section{Sacksteder 2012}

Sacksteder KA, Protopopova M, Barry CE, Andries K, Nacy CA. Discovery and development of SQ109: a new antitubercular drug with a novel mechanism of action. Future Microbiology 2012;7(7):823-37.

\section{Savic 2017}

Savic RM, Weiner M, MacKenzie WR, Engle M, Whitworth WC, Johnson JL, et al. Defining the optimal dose of rifapentine for pulmonary tuberculosis: exposure-response relations from two phase II clinical trials. Clinical Pharmacology and Therapeutics 2017;102(2):321-31. [PUBMED: 28124478]

\section{Schluger 2013}

Schluger NW. Fluoroquinolones in the treatment of tuberculosis: which is best?. American Journal of Respiratory and Critical Care Medicine 2013;188(7):768-9.

\section{Schünemann 2011}

Schünemann HJ, Oxman AD, Vist GE, Higgins JPT, Deeks JJ, Glasziou P, Guyatt GH. Chapter 12. Interpreting results and drawing conclusions. In: Higgins JP, Green S, editor(s). Cochrane
Handbook for Systematic Reviews of Interventions Version 5.1.0 (updated March 2011). The Cochrane Collaboration, 2011. Available from handbook.cochrane.org. The Cochrane Collaboration.

\section{Schünemann 2013}

Schünemann H, Brożek J, Guyatt G, Oxman A, editors. GRADE Handbook for Grading Quality of Evidence and Strength of Recommendations. Updated October 2013. Available from guidelinedevelopment.org/handbook. The GRADE Working Group.

\section{Selvakumar 2015}

Selvakumar N, Kumar V, Balaji S, Prabuseenivasan S, Radhakrishnan R, Sekar G, et al. High rates of ofloxacin resistance in Mycobacterium tuberculosis among both new and previously treated patients in Tamil Nadu, South India. PLOS One 2015;10(3):e0117421. [10.1371/ journal.pone.0117421]

\section{Sotgiu 2012}

Sotgiu G, Centis R, D'Ambrosio L, Alffenaar JWC, Anger HA, Caminero JA, et al. Efficacy, safety and tolerability of linezolid containing regimens in treating MDR-TB and XDR-TB: systematic review and meta-analysis. European Respiratory Journal 2012;40(6):1430-42.

\section{Svensson 2018}

Svensson EM, Svensson RJ, Te Brake LHM, Boeree MJ, Heinrich N, Konsten S, et al. The potential for treatment shortening with higher rifampicin doses: relating drug exposure to treatment response in patients with pulmonary tuberculosis. Clinical Infectious Diseases 2018;67(1):34-41. [DOI: 10.1093/cid/ ciy026]

\section{Tegegne 2018}

Tegegne BS, Mengesha MM, Teferra AA, Awoke MA, Habtewold TD. Association between diabetes mellitus and multi-drug-resistant tuberculosis: evidence from a systematic review and meta-analysis. Systematic Reviews 2018;7:161. [DOI: 10.1186/s13643-018-0828-0]

\section{Temple 1999}

Temple ME, Nahata MC. Rifapentine: its role in the treatment of tuberculosis. Annals of Pharmacotherapy 1999;33(11):1203-10.

\section{Wallis 2010}

Wallis RS, Jakubiec WM, Kumar V, Silvia AM, Paige D, Dimitrova D, et al. Pharmacokinetics and whole-blood bactericidal activity against Mycobacterium tuberculosis of single doses of PNU-100480 in healthy volunteers. Journal of Infectious Diseases 2010;202(5):745-51.

\section{WHO 2010}

World Health Organization. Guidelines for Treatment of Tuberculosis. 4th edition. 2010. WHO/HTM/TB/2009.420. www.who.int/tb/publications/2010/9789241547833/en/ (accessed 1 July 2017).

\section{WHO 2015}

World Health Organization. The End TB Strategy. Global Strategy and Targets for Tuberculosis Prevention, Care and 
Control After 2015. WHO/HTM/TB/2015.19. www.who.int/tb/ post2015_strategy/en/ (accessed 1 August 2017).

\section{WHO 2017}

World Health Organization. Bending the Curve Ending TB: Annual Report 2017. apps.who.int/iris/ bitstream/10665/254762/1/978929022584-eng.pdf. WHO Regional Office for South-East Asia, (accessed 12 July 2017).

\section{WHO 2018}

World Health Organization. Global Tuberculosis Report 2018. www.who.int/tb/publications/global_report/en/. Geneva: World Health Organization, (accessed prior to 24 October 2019). [Licence: CC BY-NC-SA 3.0 IGO.]

\section{Williams 2009}

Williams KN, Stover CK, Zhu T, Tasneen R, Tyagi S, Grosset JH, et al. Promising antituberculosis activity of the oxazolidinone PNU-100480 relative to that of linezolid in a murine model. Antimicrobial Agents and Chemotherapy 2009;53(4):1314-9.

\section{CHARACTERISTICS OF STUDIES}

Characteristics of included studies [ordered by study ID]

\section{Wood 2011}

Wood R, Lawn SD, Caldwell J, Kaplan R, Middelkoop K, Bekker LG. Burden of new and recurrent tuberculosis in a major South African city stratified by age and HIV-status. PLOS One 2011;6(10):e25098. [DOI: 10.1371/journal.pone.0025098]

\section{Ziganshina 2013}

Ziganshina LE, Titarenko AF, Davies GR. Fluoroquinolones for treating tuberculosis (presumed drug-sensitive). Cochrane Database of Systematic Reviews 2013, Issue 6. [DOI: 10.1002/14651858.CD004795.pub4]

\section{Zumla 2014}

Zumla Al, Gillespie SH, Hoelscher M, Philips PPJ, Cole ST, Abubakar I, et al. New antituberculosis drugs, regimens, and adjunct therapies: needs, advances, and future prospects. Lancet Infectious Diseases 2014;14(4):327-40.

* Indicates the major publication for the study

\section{Gillespie 2014}

Methods

Study design: multi-centre, randomized, parallel-group, double-blind (participant, care provider, investigator, outcomes assessor), 3-armed, placebo-controlled, non-inferiority trial

Study period: January 2008 to February 2014

Recruitment sites: 47 sites in 9 countries

Countries where the trial was undertaken: South Africa, India, Tanzania, Kenya, Thailand, Malaysia, Zambia, China, Mexico

Length of follow-up: 18 months after randomization (1 year after treatment completion)

Participants

No. of participants randomized: 1931

Interventions: 1291 (636 to ethambutol group; 655 to isoniazid group)

Control: 640 .

Age: > 35 years, $37 \%$ in isoniazid group, $39 \%$ in ethambutol group, and $40 \%$ in control group

Gender: male $70 \%$ in ethambutol group, $68 \%$ in isoniazid group, $70 \%$ in control group

Inclusion criteria:

- 2 sputum specimens positive for tubercle bacilli on direct smear microscopy, of which 1 was confirmed by the REMoxTB study laboratory at the local laboratory

- No history of previous anti-tuberculosis chemotherapy

- Aged 18 years and older

- Firm home address that is readily accessible for visiting and willingness to inform the study team of any change in address and follow-up period

- Agreement to participate in the study and to give a sample of blood for HIV testing

- Negative pregnancy test (women of childbearing potential)

- Pre-menopausal women must be using a barrier form of contraception or must be surgically sterilized or have an IUCD in place 
- Laboratory parameters performed at least 14 days before enrolment

* Serum aspartate transaminase (AST) and alanine transaminase (ALT) activity less than 3 times upper limit of normal

* Serum total bilirubin level less than 2.5 times upper limit of normal

* Creatinine clearance $(\mathrm{CrCl})$ level greater than $30 \mathrm{~mL} / \mathrm{min}$

* Haemoglobin level at least $7.0 \mathrm{~g} / \mathrm{dL}$

* Platelet count at least $50 \times 10^{9}$ cells/L

* Serum potassium greater than $3.5 \mathrm{mmol} / \mathrm{L}$

\section{Exclusion criteria:}

- Patients unable to take oral medication

- Previously enrolled in this study

- Receiving any investigational drug in the past 3 months or an antibiotic active against $M$ tuberculosis

- Pregnancy or breastfeeding

- Any condition that may prove fatal during the first 2 months of the study period

- Severe tuberculosis with high risk of a poor outcome (e.g. meningitis)

- Pre-existing condition likely to prejudice the response to, or assessment of, treatment; a condition likely to lead to uncooperative behaviour

- Contraindication to any medications in the study regimens

- Congenital or sporadic cardiac syndrome or taking medications that could result in QTc prolongation

- Patients already receiving antiretroviral therapy

- Weight less than $35 \mathrm{~kg}$

- HIV infection with CD4 count less than 250 cells/ $\mu \mathrm{L}$

- End-stage liver failure (class Child-Pugh C)

- Patients whose initial isolate was shown to be multiple drug resistant or monoresistant to rifampicin, or to any fluoroquinolone

Proportion with HIV seropositivity: $7 \%$ overall (and in intervention and control groups)

Proportion with cavitation: $71 \%$ overall (69\% and $70 \%$ in intervention groups and $72 \%$ in control groups)

Baseline drug resistance: isoniazid: $7 \%$ overall ( $6 \%$ in control arm and $7 \%$ in each intervention arm); pyrazinamide: $2 \%$ overall ( $1 \%$ in each intervention arm)

Interventions

Interventions: 4-month (17-week) ATT regimen

Isoniazid group (moxifloxacin for 17 weeks substituting ethambutol): $\mathrm{N}=655$; 568 eligible, 514 completed ( $78 \%$ of those randomized; $91 \%$ of those eligible)

8 weeks of moxifloxacin, isoniazid, rifampicin, pyrazinamide, + ethambutol placebo administered daily, followed by

9 weeks of moxifloxacin, isoniazid, and rifampicin, followed by 9 weeks of isoniazid and rifampicin placebo

Ethambutol group (moxifloxacin for 17 weeks substituting isoniazid): $\mathrm{N}=636$; 551 eligible, 524 completed ( $82 \%$ of those randomized; $91 \%$ of those eligible)

8 weeks of moxifloxacin, ethambutol, rifampicin, pyrazinamide + isoniazid placebo administered daily, followed by

9 weeks of moxifloxacin and rifampicin + isoniazid placebo daily, followed by

9 weeks of isoniazid and rifampicin placebo

Control: 6-month (26-week) ATT regimen: $\mathrm{N}=640$; 555 eligible, 510 completed ( $80 \%$ of those randomized; $92 \%$ of those eligible) 
8 weeks of isoniazid, rifampicin, ethambutol, pyrazinamide, and moxifloxacin placebo given daily, followed by

9 weeks of isoniazid, rifampicin, and moxifloxacin placebo given daily, followed by

9 weeks of isoniazid and rifampicin

Dosage: Moxifloxacin $400 \mathrm{mg}$, isoniazid $300 \mathrm{mg}$; rifampicin, ethambutol, and pyrazinamide were dosed based on weight

Outcomes

Outcomes reported and used in this review:

- Relapse within 18 months after randomization (relapse strains were those shown to be identical on 24-locus MIRU analysis)

- Death from any cause

- Rates of treatment discontinuation

- Sputum smear/culture positivity at 8 weeks

- Proportion with bacteriologically or clinically defined failure

- Serious adverse events

- Other adverse events

\section{Outcomes sought but not reported:}

- Development of secondary drug resistance to anti-tuberculosis drugs, identified by drug susceptibility testing

\section{Outcomes reported but not used in this review:}

- Composite unfavourable outcome (clinical or bacteriologic failure or relapse within 18 months after randomization) (non-inferiority was defined as a between-group difference of less than 6 percentage points in the upper boundary of the 2 -sided $97.5 \%$ Wald confidence interval for the difference in proportion of patients with an unfavourable outcome)

- Re-treated for tuberculosis

- Time to first culture-negative sputum

- Pharmakokinetic data (substudy reported separately) cies and local agencies and institutions in participating countries). Bayer Healthcare donated moxifloxacin and Sanofi donated rifampicin

Treatment supervision: treatment was given daily and was observed according to guidelines at the study site

Follow-up method: following screening and baseline visits, there were 8 weekly visits followed by 8 visits until 18 months after randomization. Safety analysis was performed at the screening visit and thereafter at weeks $2,8,12$, and 17

Trial registration ID: NCT00864383 (retrospectively registered: registered March 2009; study start January 2008)

\section{Acronym: REMoxTB}

Comment: data from both moxifloxacin-containing shorter regimens were combined and compared with data from the standard treatment regimen

\section{Risk of bias}


Gillespie 2014 (Continued)

Random sequence genera- Low risk tion (selection bias)
Quote from report: "Randomization was performed with the use of lists with blocks of variable sizes that were stratified according to the patient weight group and study centre"

\section{Allocation concealment Low risk} (selection bias)
Quote from report: "During randomization, patients were assigned a unique study number selected sequentially from the appropriate randomization list that corresponded to the treatment pack allocated"

Quote from report: "Only statisticians who were responsible for preparing the reports for the independent data and safety monitoring committee and essential manufacturing and distribution staff members had access to the list of identifiers matched to the intervention"

$\begin{array}{ll}\begin{array}{l}\text { Blinding of participants } \\ \text { and personnel (perfor- }\end{array} & \text { Low risk } \\ \begin{array}{l}\text { mance bias) } \\ \text { All outcomes }\end{array} & \begin{array}{l}\text { Quote from study protocol: "This will be a blinded study with matching place- } \\ \text { bo for each of the study medicines except for pyrazinamide" }\end{array} \\ & \begin{array}{l}\text { Quote from trial registration document: "Masking: quadruple (participant, care } \\ \text { provider, investigator, outcomes assessor)" }\end{array}\end{array}$

Blinding of outcome as- Low risk sessment (detection bias) All outcomes
Quote from trial registration document: "Masking: quadruple (participant, care provider, investigator, outcomes assessor)"

Incomplete outcome da- Low risk

ta (attrition bias): At the end of ATT (Treatment failure, positive sputum culture, treatment discontinuation, adverse events)
Quote from report: "Of the 1931 patients who underwent randomization, 89\% in the isoniazid group, $92 \%$ in the ethambutol group, and $89 \%$ in the control group met the requirements for treatment adherence, which was based on receipt of approximately $80 \%$ of the assigned regimen"

Comment: the modified-intention-to-treat analysis used included $87 \%$ of those randomized to the combined moxifloxacin-containing ATT regimens and $87 \%$ of those randomized to standard regimens. A sensitivity analysis included $94 \%$ of those randomized to both regimens
Incomplete outcome da- Low risk ta (attrition bias): At the end of follow-up (Relapse, deaths)
Of those randomized, $91 \%$ of those allotted to the 2 moxifloxacin combination therapy arms and $92 \%$ allotted to control treatment were included in the modified intention-to-treat analyses. Results of the per-protocol and modified intention-to-treat analyses were consistent

Selective reporting (re- Low risk
porting bias)
Although the trial was retrospectively registered, all pre-stated outcomes listed in the trial registration document and protocol were published with no evidence of selective reporting

Other bias Low risk

Quote from report: "Bayer Healthcare donated moxifloxacin, and Sanofi donated rifampin. Neither company had any role in the study design, data accrual, data analysis, or manuscript preparation. Representatives of Bayer Healthcare reviewed the manuscript but did not suggest revisions"

Study period: started May 2004 for an anticipated duration of 5 years; terminated early (between February and October 2006) due to high recurrence rates in the shorter treatment arms

Recruitment sites: Chennai and Madurai

Country where the trial was undertaken: India 
Length of follow-up: 24 months after treatment completion

Participants

No. of participants randomized: 429 (of 1200 anticipated)

Intervention groups: 259 (gatifloxacin regimen 141; moxifloxacin regimen 118)

Control group: 170

Age: $<40$ years $72 \%$ (gatifloxacin 66\%; moxifloxacin $77 \%$; control $73 \%$ )

Gender: male $74 \%$ (gatifloxacin 76\%; moxifloxacin $72 \%$; control $72 \%$ )

\section{Inclusion criteria:}

- Adult patients 18 years or older with newly diagnosed pulmonary tuberculosis with at least 2 positive sputum cultures

- Resident within a designated study area and permitted home visits

\section{Exclusion criteria:}

- Those with previous treatment for tuberculosis exceeding 30 days, weighing $<30 \mathrm{~kg}$, pregnant or lactating women

- Those with concomitant diabetes mellitus, severe systemic hypertension, epilepsy, serious forms of extrapulmonary tuberculosis, or HIV infection

Proportion with HIV seropositivity: nil (excluded)

Proportion with cavitation: not reported

Baseline drug resistance: isoniazid $7 \%$ overall (gatifloxacin 4\%; moxifloxacin $1.2 \%$; control $12 \%$ ); rifampicin $0.2 \%$ overall (moxifloxacin $1 \%$ ); ofloxacin $1.7 \%$ overall (gatifloxacin $2 \%$; control $3 \%$ ); isoniazid and ethambutol $0.4 \%$ overall ( $1 \%$ in each intervention arm); isoniazid and ofloxacin $0.2 \%$ overall (control $1 \%)$

Interventions

Interventions: 4-month ATT regimens

Gatifloxacin regimen (gatifloxacin replacing ethambutol): $\mathrm{N}=141 ; 136$ eligible, 131 completed (93\% of those randomized; $96 \%$ of those eligible)

2 months of gatifloxacin, isoniazid, rifampicin, and pyrazinamide thrice weekly, followed by

2 months of gatifloxacin, isoniazid, and rifampicin thrice weekly

Moxifloxacin regimen (moxifloxacin replacing ethambutol): $\mathrm{N}=118 ; 115$ eligible, 113 completed (96\% of those randomized; $98 \%$ of those eligible)

2 months of moxifloxacin, isoniazid, rifampicin, and pyrazinamide thrice weekly, followed by

2 months of moxifloxacin, isoniazid, and rifampicin thrice weekly

Control: 6-month ATT regimen: $\mathrm{N}=170$; 165 eligible, 159 completed (94\% of those randomized; $96 \%$ of those eligible)

2 months of ethambutol, isoniazid, rifampicin, and pyrazinamide thrice weekly, followed by

4 months of isoniazid and rifampicin thrice weekly

Dosage: gatifloxacin or moxifloxacin $400 \mathrm{mg}$, rifampicin 450 or $600 \mathrm{mg}$, depending on body weight (<60 $\mathrm{kg}$ or $\geq 60 \mathrm{~kg}$ ), pyrazinamide $1500 \mathrm{mg}$, and isoniazid $600 \mathrm{mg}$

- Recurrence of tuberculosis among those with a favourable response at the end of treatment

- Death from any cause 
Jawahar 2013 (Continued)

- Rates of treatment discontinuation

- Sputum smear/culture positivity at 8 weeks

- Proportion with bacteriologically or clinically defined failure

- Serious adverse events

- Other adverse events

- Development of secondary drug resistance to anti-tuberculosis drugs, identified by drug susceptibility testing

Follow-up method: a physician examined the patient every month and recorded adherence to treatment, any adverse drug reactions, and the clinical response. Sputum specimens were examined every month by microscopy and culture: 2 ( 2 overnight and 1 spot) during the treatment phase, and 2 ( 1 overnight and 1 spot) during the follow-up phase

Treatment supervision: directly observed, thrice-weekly treatment in all arms

Trial registration ID: CTRI/2012/10/003060; retrospectively registered (trial commenced May 2004; trial registered 15/10/2012, after termination)

Comment: the data safety monitoring board recommended termination of both intervention arms in 2006 due to high tuberculosis recurrence rates in the 2 arms compared to the standard 6-month regimen

\section{Risk of bias}

Bias Authors' judgement Support for judgement

Random sequence genera- Low risk tion (selection bias)
Quote from report: "Restricted random allocation sequences were generated by a biostatistician using random number tables, separately for the two strata and sealed envelopes were used to assign regimens"

Quote from trial registration document: "Stratified block randomization"
Allocation concealment High risk (selection bias)
Quote from report: "Patients were enrolled by the physicians, and when ready for allocation, the biostatistician drew the regimen from sealed envelopes. Allocation was stratified on sputum smear grading and extent of lesions in chest $x$ ray"

Quote from report: "The study design envisaged enrolling 400 patients in each arm in a 1:1:1 ratio. However, due to the non-availability of one of the test drugs (M), patients were enrolled initially in a $1: 1$ ratio in the $G$ and control regimen arms commencing in May 2004. Subsequently, when $M$ became available (May 2005), patients were enrolled to the $G, M$, and control regimen arms in a $1: 2: 1$ ratio to compensate for the delay in recruiting to the moxifloxacin arm at the onset"

Quotes from correspondence with study authors: "When the first patient on Moxifloxacin was allocated, there were 110 patients randomised to the Gatifloxacin regimen and 110 to the Control regimen. The last patient was allocated to the Gatifloxacin regimen on 3 February 2006"

Comment: alteration of recruitment ratios raises serious concerns that allocation concealment was compromised. Even though biostatisticians implemented allocation after clinicians confirmed eligibility, by the time the first patient was recruited to the moxifloxacin regimen in May 2005, 110 allocated to the gatifloxacin regimen ( $80 \%$ of 136 eligible among those finally recruited), and 110 allocated to the control regimen ( $67 \%$ of the 165 eligible) had already been recruited. This would have alerted investigators that most of those to be recruited over the following year would be allocated to the moxifloxacin regimen. In addition, premature termination of the trial, combined with the al- 
teration in allocation ratios, appears to have led to imbalance in the numbers recruited to the gatifloxacin (141), moxifloxacin (118), and control (170) regimens that is not explained, given that block randomization was used. There were also baseline imbalances in proportions resistant at baseline to any of the anti-tuberculosis drugs tested (6\%, $3 \%$, and $16 \%$ respectively)

\begin{tabular}{|c|c|c|}
\hline $\begin{array}{l}\text { Blinding of participants } \\
\text { and personnel (perfor- } \\
\text { mance bias) }\end{array}$ & Low risk & $\begin{array}{l}\text { This was an open-label trial; given the likelihood that allocation concealment } \\
\text { was compromised, treating personnel may have had knowledge of allocation. } \\
\text { However, it is unlikely that this led to performance bias }\end{array}$ \\
\hline
\end{tabular}

All outcomes

Blinding of outcome as- Low risk sessment (detection bias) All outcomes

\begin{abstract}
Quote from report: "Sputum specimens were given identification laboratory numbers, and bacteriological investigations were carried out by technicians who were blinded to the clinical status of the patient and the regimen. ECG was done every month"

Comment: although bacteriologic outcomes were done blind to treatment allocation, clinical efficacy and safety outcomes were undertaken by study personnel. But results at the time of termination favouring the standard and not experimental interventions suggest that detection bias was unlikely
\end{abstract}

\begin{abstract}
Incomplete outcome da- Low risk ta (attrition bias): At the end of ATT (Treatment failure, positive sputum culture, treatment discontinuation, adverse events)

In the combined fluoroquinolone arms, 228 of 259 (88\%) completed treatment compared to 152 of $170(89 \%)$ in the control arms. Study results did not differ between per-protocol and modified intention-to-treat analyses (that excluded only late-screening failures). The modified-ITT analysis included 97\%, 98\%, and $97 \%$ of those in the gatifloxacin, moxifloxacin, and standard ATT arms, respectively. The results of both analyses were consistent
\end{abstract}

Incomplete outcome da- Low risk ta (attrition bias): At the end of follow-up (Relapse, deaths)
In the combined fluoroquinolone arms, 230 of 259 (89\%) were assessed for tuberculosis recurrence compared to 154 of 170 (91\%) in the control arm. Perprotocol and modified intention-to-treat analyses did not significantly alter the results. Early termination led to recruitment of only a third of the estimated 1200 participants required to prove equivalence, but although this reduces the power of the trial to detect equivalence, lack of differential attrition, with similar reasons for exclusion, is unlikely to affect the reported relative effect estimates

\begin{tabular}{|c|c|c|}
\hline $\begin{array}{l}\text { Selective reporting (re- } \\
\text { porting bias) }\end{array}$ & Low risk & $\begin{array}{l}\text { Although the trial was retrospectively registered, stated outcomes in trial reg- } \\
\text { istry documents and in the online study protocol were available in the trial re- } \\
\text { port and do not suggest selective reporting }\end{array}$ \\
\hline
\end{tabular}

Other bias Low risk

The trial was terminated early at the recommendation of the Data Safety Monitoring Board after an interim analysis showed high recurrence rates in the fluoroquinolone arms compared to the control arm. Because this was a planned interim analysis, it is unlikely to have introduced bias, other than that discussed under allocation concealment

\section{Jindani 2014}

Methods

Study design: randomized, multi-centre, parallel-group, open-label, 3-arm, active-controlled, equivalence trial

Study period: August 15, 2008, and August 1, 2011

Recruitment sites: Worcester, Johannesburg, Harare, Marondera, Francistown, and Macha

Countries where the trial was undertaken: Botswana, South Africa, Zambia, and Zimbabwe 
Length of follow-up: 18 months after randomization in 86\%; in Botswana and South Africa, $6 \%$ of those randomized in the last 6 months of enrolment were followed up for 12 to 15 months and $8 \%$ for 15 to 18 months

Participants

No. of participants randomized: 827 (of the estimated sample of 1095)

Interventions: 275 in 4-month regimen (277 in 6-month regimen)

Control group: 275 in the control regimen

Age: 18 to 34 years $61 \%$ in the control regimen and $68 \%$ in the 4-month regimen

Gender: male $64 \%$ in the control regimen, $63 \%$ in the 4-month regimen

Inclusion criteria:

- Newly diagnosed pulmonary tuberculosis

- 2 sputum specimens positive for tubercle bacilli on direct smear microscopy

- Either no previous anti-tuberculosis chemotherapy or less than 2 weeks of previous chemotherapy at enrolment

- Aged 18 years and older

- Firm home address that is readily accessible for visiting and intending to remain there or within the recruitment area for the entire treatment and follow-up period

- Willing to agree to participate in the study and to give a sample of blood for HIV testing (and in Botswana to have HIV status disclosed to them)

- Pre-menopausal women must be using a barrier form of contraception or must be surgically sterilized or have an IUCD in place for the duration of the treatment phase

\section{Exclusion criteria:}

- Any condition (except HIV infection) that may prove fatal during the study period

- Tuberculosis meningitis

- Pre-existing non-tuberculous disease likely to prejudice the response to, or assessment of, treatment (e.g. insulin-dependent diabetes, liver or kidney disease, blood disorders, peripheral neuritis)

- Female and known to be pregnant or breastfeeding

- Condition likely to lead to uncooperative behaviour such as psychiatric illness or alcoholism

- Contraindications to any medications in the study regimens

- History of prolonged QTC syndrome or current or planned therapy with quinidine, procainamide, amiodarone, sotalol, disopyramide, ziprasidone, or terfenadine during the intensive phase of tuberculosis therapy

- Haemoglobin $<7 \mathrm{~g} / \mathrm{L}$

- AST or ALT > 5 times the upper range

- Creatinine clearance $<30 \mathrm{~mL} / \mathrm{min}$

- History of seizures

- HIV positive with CD4 count less than $150 / \mathrm{mm}^{3}$

- Weight $<35 \mathrm{~kg}$

- Already receiving anti-retroviral therapy (ART)

Proportion with HIV seropositivity: $32 \%$ in the control regimen and $28 \%$ in the 4-month regimen

Proportion with cavitation: $67 \%$ in the control regimen and $65 \%$ in the 4-month regimen

Baseline drug resistance: excluded people resistant to isoniazid, rifampicin, or moxifloxacin

Interventions Interventions:

4-month (17-week) ATT regimen: moxifloxacin replacing isoniazid throughout with twice-weekly administration in continuation phase + rifapentine twice weekly replacing rifampicin in continuation 
phase: $\mathrm{N}=275$ randomized; 239 eligible, 165 completed ( $60 \%$ of those randomized; $69 \%$ of those eligible)

2 months ( 8 weeks) of ethambutol, moxifloxacin, rifampicin, and pyrazinamide administered daily, followed by

2 months (9 weeks) of moxifloxacin and rifapentine administered twice weekly

6-month (26-week) ATT regimen: moxifloxacin replacing isoniazid throughout with weekly rifapentine + weekly moxifloxacin in the 4-month continuation phase

2 months (8 weeks) of ethambutol, moxifloxacin, rifampicin, and pyrazinamide administered daily, followed by

4 months (18 weeks) of rifapentine and moxifloxacin once a week

Control: 6-month (26-week) ATT regimen: $\mathrm{N}=275$ randomized; 240 eligible, 163 completed (59\% of those randomized; $68 \%$ of those eligible)

2 months ( 8 weeks) of isoniazid, rifampicin, ethambutol, and pyrazinamide administered daily, followed by 18 weeks of isoniazid and rifampicin daily

Dosage: moxifloxacin $400 \mathrm{mg}$; rifapentine $900 \mathrm{mg}$ in the 4-month treatment arm (and $1200 \mathrm{mg}$ in the 6month arm). All doses given were based on the weight of the patient

Outcomes

\section{Outcomes reported and used in this review:}

- Relapse after treatment

- Death from any cause

- Failure to complete treatment

- Treatment failure

- Acquired drug resistance

- Serious adverse events

\section{Outcomes sought for this review and not reported:}

- Sputum positive smear/culture at 8 weeks (disaggregated data from the 2 moxifloxacin arms not reported)

\section{Outcomes reported and not used in this review:}

- Culture results at end of follow-up

- ART start times in HIV-infected people

- Adherence trial medications were donated by Sanofi, Genus Pharmaceuticals, and Sandoz

\section{Follow-up method}

Patients were followed-up monthly up to 12 months after randomization and thereafter once in 3 months until 18 months. Two sputum samples were collected before treatment initiation for smear and culture, and 1 sample was collected monthly for 12 months and then again at 15 months and 18 months of follow-up

Treatment supervision: treatment was directly observed in all participants in the intensive treatment phase. Drugs were taken under the supervision of a relative or another designated person in the 18week continuation phase in the control arm. Moxifloxacin and rifapentine treatment was supervised at the treatment facility twice weekly for the 9-week continuation phase

Trial registration ID: ISRCTN44153044 (prospectively registered)

Acronym: RIFAQUIN 


\section{Risk of bias}

\begin{tabular}{lll}
\hline Bias & Authors' judgement & Support for judgement \\
\hline $\begin{array}{l}\text { Random sequence genera- } \\
\text { tion (selection bias) }\end{array}$ & Low risk & $\begin{array}{l}\text { Quote from report: "A randomized allocation sequence was generated for each } \\
\text { study centre with the use of blocks of varying size by an independent statisti- } \\
\text { cian based at the MRC CTU" }\end{array}$ \\
\hline $\begin{array}{l}\text { Allocation concealment } \\
\text { (selection bias) }\end{array}$ & $\begin{array}{l}\text { Quote from updated protocol: "Sealed opaque envelopes containing the treat- } \\
\text { ment allocation slips will be held by the pharmacist. When a patient is found to } \\
\text { be eligible their details will be entered on the enrolment log by the designat- } \\
\text { ed member of the clinic team against the next available study number. These } \\
\text { patient details and the study number will be entered on to the patient's pre- } \\
\text { scription. This will be taken to the pharmacy and the patient details entered } \\
\text { onto the pharmacy register by the pharmacist against the next study number } \\
\text { which will act as a check that the correct (next available) study number had } \\
\text { been used. The pharmacist will then take the envelope corresponding to the } \\
\text { study number and reveal the treatment allocation which will be written on the } \\
\text { allocation slip. This will then be attached to the prescription and kept in the } \\
\text { patient's Trial folder or other appropriate place and the designated member of } \\
\text { the clinic team made aware of the treatment allocation" }\end{array}$
\end{tabular}

$\begin{array}{ll}\text { Blinding of participants } & \text { Low risk } \\ \begin{array}{l}\text { and personnel (perfor- } \\ \text { mance bias) }\end{array} & \begin{array}{l}\text { This was an open-label trial. The treating team was aware of allocated treat- } \\ \text { ments. However, this does not seem to have influenced drug administration or } \\ \text { use of co-interventions }\end{array}\end{array}$
use of co-interventions

All outcomes

Blinding of outcome as- Low risk sessment (detection bias)

All outcomes
Quote from report: "Apart from the statisticians reporting to the data and safety monitoring committee, the staff at St. George's and at the MRC CTU were unaware of treatment assignment except when a lack of awareness would have been unethical (e.g., in some discussions of serious adverse events). Participating laboratories were unaware of treatment assignment throughout the study"

Comment: although treatment allocation before the start of the trial was concealed, the clinical team evaluating participants for efficacy and safety outcomes was aware of treatment allocation. However, laboratory assessments were objective and clinical outcomes were mostly based on objective assessments

\section{Incomplete outcome da- Low risk}

ta (attrition bias): At the end of ATT (Treatment failure, positive sputum culture, treatment discontinuation, adverse events)
Although overall attrition was over $30 \%$, there was no differential attrition in the 4-month arm (31\%) versus the control arm (32\%). In the sensitivity analysis in the supplementary table, S1 attrition was $13 \%$ in each arm. We do not think this is likely to alter the estimates of relative effects

\begin{tabular}{|c|c|c|}
\hline $\begin{array}{l}\text { Incomplete outcome da- } \\
\text { ta (attrition bias): At the } \\
\text { end of follow-up (Relapse, } \\
\text { deaths) }\end{array}$ & Low risk & $\begin{array}{l}\text { Modifed intention-to-treat and per-protocol analyses presented in Table } 2 \text { of } \\
\text { the main report and in the sensitivity analyses in Table S1 in the online supple- } \\
\text { mentary appendix do not indicate that bias due to differential attrition is likely } \\
\text { to have affected the estimates of relative effects }\end{array}$ \\
\hline
\end{tabular}
deaths)

\begin{tabular}{|c|c|c|}
\hline $\begin{array}{l}\text { Selective reporting (re- } \\
\text { porting bias) }\end{array}$ & Low risk & $\begin{array}{l}\text { This trial was prospectively registered, and protocol amendments and report- } \\
\text { ing of results do not indicate selective reporting }\end{array}$ \\
\hline Other bias & Low risk & $\begin{array}{l}\text { Quote from report: "Some of the trial medications were donated by Sanofi, } \\
\text { Genus Pharmaceuticals, and Sandoz, and a representative of Sanofi was a } \\
\text { non-voting observer at meetings of the steering committee, but none of these }\end{array}$ \\
\hline
\end{tabular}


Study period: June 2005 to April 2011

Recruitment sites: Conakry, Cotonou, Dakar, Durban, Nairobi

Countries where the trial was undertaken: Benin, Guinea, Kenya, Senegal, South Africa

Length of follow-up: 24 months

Participants

\section{No of participants randomized: 1836}

\section{Intervention: 917}

\section{Control: 919}

Age: mean age intervention 30.9 years, control 30.6 years

Gender: male: Intervention $73 \%$, control $72 \%$

\section{Inclusion criteria:}

- Aged 18 to 65 years (both inclusive) and weighing between $38 \mathrm{~kg}$ and $80 \mathrm{~kg}$

- Recently diagnosed, microscopically proven, pulmonary tuberculosis, defined as 2 consecutively positive sputum smears, of which 1 must be equal to or exceed grade 1

- Findings in medical history and physical examination not exceeding grade 2 according to the Division of Microbiology and Infectious Disease grading system tables (DMID)

- Voluntarily signed informed consent to participate in the study

- Females of childbearing potential must have a confirmed negative pregnancy test at the screening visit and must employ an effective and acceptable method of birth control during treatment

- Laboratory values that do not exceed grade 2 using the Division of Microbiology and Infectious Disease grading system (DMID) other than for glycaemia, haemoglobin, and potassium levels

\section{Exclusion criteria:}

- Patients with a history of tuberculosis treatment within the last 3 years

- Concomitant infection requiring additional anti-infective treatment (especially antiretroviral medication - ARV)

- HIV-infected patients with WHO stage 3 infection (except those presenting with only the "loss of weight $>10 \%$ body weight" criterion) and all patients at WHO stage 4 (see Appendix 5)

- History of diabetes mellitus (DM) or non-insulin-dependent diabetes mellitus (NIDDM) requiring treatment or diet. Additionally, patients who have a fasting glucose level less than $70 \mathrm{mg} / \mathrm{dL}(3.9 \mathrm{mmol} / \mathrm{L})$ or above $115 \mathrm{mg} / \mathrm{dL}(6.4 \mathrm{mmol} / \mathrm{L})$ at screening will be excluded

- Recreational drug abuse and alcohol abuse that, in the opinion of the investigator, could prejudice the conduct of the study in that patient

- History of drug hypersensitivity and/or active allergic disease

- Impaired renal, hepatic, or gastric function that may, in the opinion of the investigator, interfere with drug absorption, distribution, metabolism, or elimination

- Any other findings in medical history and physical examination exceeding grade 2 in the DMID grading system tables 
- Patient using the following therapies:

* Other antibiotics with known anti-tuberculosis activity (i.e. ofloxacin, moxifloxacin, kanamycin)

* Drugs known to prolong the QT interval (i.e. anti-arrhythmics, psychotropics (phenothiazines, tricyclics, tetracyclics), erythromycin, pentamidine, and halofantrine)

* Drugs known to give photosensitivity reactions

* Receiving oral corticosteroids for longer than 2 weeks immediately before inclusion

* Use of antacids containing aluminium or magnesium salts or sucralfate

* Digoxin

* Drugs that are eliminated via tubular secretion (e.g. probenecid, cimetidine, ranitidine)

- Pregnant or lactating women

- Patients with congenital QT interval prolongation $>480 \mathrm{~ms}$

- Patients with clinically significant bradycardia (40 beats/min)

- Baseline laboratory values exceeding grade 2 using the Division of Microbiology and Infectious Disease grading system (DMID) except glycaemia value as previously stated, haemoglobin, and hypokalaemia for which the limit values are as follows: potassium $<3.0 \mathrm{mEq} / \mathrm{L}$ ( $>$ grade 1 ), haemoglobin $<6.5 \mathrm{~g} / \mathrm{dL}$

- Any other finding considered by the investigator as compromising the participation of the patient in the trial

- Any condition rendering the patient unable to understand the nature, scope, and possible consequences of the study and to provide consent

- Participation in another drug trial within the 3 months before the screening visit

Proportion with HIV seropositivity: $19 \%$ in the control regimen and $18 \%$ in the 4-month regimen

Proportion with cavitation: $50 \%$ in the control regimen and $52 \%$ in the 4-month regimen

Baseline drug resistance: excluded people with rifampicin resistance and MDR-TB; isoniazid resistance: (gatifloxacin 8.5\%; control 6.6\%)

Interventions

Intervention: 4-month ATT regimen

Gatifloxacin: gatifloxacin replacing ethambutol in intensive and continuation phases: $\mathrm{N}=917$ randomized; 791 eligible, 651 included in per-protocol analysis ( $71 \%$ of those randomized, $82 \%$ of those eligible)

2 months of gatifloxacin, isoniazid, rifampicin, and pyrazinamide given daily, followed by

2 months of gatifloxacin, isoniazid, and rifampicin

Control: 6-month ATT regimen ( $\mathrm{N}=919$ randomized; 784 eligible, 601 included in per-protocol analysis ( $65 \%$ of those randomized; $77 \%$ of those eligible)

2 months of ethambutol, isoniazid, rifampicin, and pyrazinamide, followed by

4 months of isoniazid and rifampicin

\section{Dosage:}

Fixed-dose combination tablets of isoniazid-rifampin, isoniazid-rifampin-pyrazinamide, or isoniazid-rifampin-pyrazinamide-ethambutol were used wherever needed. Gatifloxacin was given at a dose of $400 \mathrm{mg}$. Other drugs were given in weight-based doses ( $<50 \mathrm{~kg}, \geq 50 \mathrm{~kg}$ )

\section{Outcomes reported and used in this review:}

- Recurrence (relapse or reinfection)

- Death from any cause

- Treatment failure (at 4 months or 6 months)

- Sputum positive smear/culture at 8 weeks

- Treatment discontinuation

- Serious adverse events 
Outcomes sought for this review but not reported:

- Acquired drug resistance

Outcomes reported and not used in this review:

- Unfavourable outcome by 24 months after treatment (composite of treatment failure, recurrence, death, or withdrawal)

- Unfavourable outcome at 18 months after randomization

- Time to an unfavourable outcome after treatment

- Pharmacokinetic/pharmacodynamic data (published separately)

Notes

Funding: Institut de Recherche pour le Développement (IRD) (on behalf of the OFLOTUB Consortium), World Health Organization (WHO). Lupin Pharmaceuticals provided study medicines

Follow-up method: trial drugs were administered orally under supervision 6 days a week during the intensive phase and were provided every 2 weeks in the continuation phase. Two sputum samples were obtained for smear examination, solid culture, and drug sensitivity tests at baseline and at all subsequent visits. Electrocardiograms (ECGs) were done at baseline, between 1 and 5 hours after drug intake, at 4 weeks, at 8 weeks, and at end of treatment

Treatment supervision: trial drugs were given orally, 6 days a week under direct observation supervision during intensive phase and were provided every 2 weeks in the continuation phase to a supervisor who ensured treatment was taken. Adherence was assessed by pill count that remained in the weekly treatment boxes

Trial Registration ID: NCT00216385 (retrospectively registered: September 2005)

Acronym: OFLOTUB/gatifloxacin

\section{Risk of bias}

\section{Bias \\ Authors' judgement Support for judgement}

Random sequence genera- Low risk tion (selection bias)
Quote from report: "Patients were randomly assigned, in a 1:1 ratio with stratification according to country, to either a gatifloxacin-containing regimen (experimental group) or the 6-month standard treatment (control group)"

Quote from protocol: "Randomization lists, stratified by study site and indicating a randomization number and which treatment is to be given, will be produced prior to the start of the trial by the medical statistician in London"

\begin{tabular}{|c|c|c|}
\hline $\begin{array}{l}\text { Allocation concealment } \\
\text { (selection bias) }\end{array}$ & Low risk & $\begin{array}{l}\text { Quote from protocol:"The Code for each individual will be provided in sepa- } \\
\text { rate sealed envelopes and assigned to individuals in the order in which they } \\
\text { are enrolled in the study" }\end{array}$ \\
\hline $\begin{array}{l}\text { Blinding of participants } \\
\text { and personnel (perfor- } \\
\text { mance bias) } \\
\text { All outcomes }\end{array}$ & Low risk & $\begin{array}{l}\text { Quote from protocol: "It must be noted that management of patients cannot } \\
\text { be blinded, because of the difference in treatment length, but steps will be } \\
\text { taken to ensure equal management and follow-up of both treatment arms" }\end{array}$ \\
\hline $\begin{array}{l}\text { Blinding of outcome as- } \\
\text { sessment (detection bias) } \\
\text { All outcomes }\end{array}$ & Low risk & $\begin{array}{l}\text { Quote from protocol: "Lastly, when patients recruited in the trial come to the } \\
\text { clinic with a suspicion of relapse, the treatment they received will be blinded } \\
\text { to the physician examining them" } \\
\text { Quote from protocol: "Laboratory technicians will be blinded to the origin of } \\
\text { each sample, ensuring unbiased assessment of endpoints" }\end{array}$ \\
\hline
\end{tabular}


Merle 2014 (Continued)

Incomplete outcome data (attrition bias): At the end of ATT (Treatment failure, positive sputum culture, treatment discontinuation, adverse events)
Low risk Althought $35 \%$ of those eligible after randomization in the control arm and $29 \%$ in the intervention arm were excluded from the per-protocol analyses, modified intention-to-treat analyses included $86 \%$ of those randomized to each arm. Results of modified intention-to treat analyses and per-protocol analyses were consistent and did not suggest that differential attrition significantly biased the relative estimates of effects

\begin{tabular}{|c|c|c|}
\hline $\begin{array}{l}\text { Incomplete outcome da- } \\
\text { ta (attrition bias): At the } \\
\text { end of follow-up (Relapse, }\end{array}$ & Low risk & $\begin{array}{l}\text { Quote from report: "The cumulative percentage of patients retained in the } \\
\text { experimental and control groups, respectively, was } 93.5 \% \text { and } 93.4 \% \text { by } 52 \\
\text { weeks, } 91.0 \% \text { and } 89.6 \% \text { by } 78 \text { weeks, and } 87.5 \% \text { and } 82.7 \% \text { by } 94 \text { weeks" }\end{array}$ \\
\hline
\end{tabular}
deaths)

Comment: there was differential attrition over 24 months, but results of the per-protocol and intention-to-treat analyses were consistent

\begin{tabular}{lll}
\hline $\begin{array}{l}\text { Selective reporting (re- } \\
\text { porting bias) }\end{array}$ & Low risk & $\begin{array}{l}\text { The trial was retrospectively registered. However all outcomes in the registra- } \\
\text { tion documents and changes in the protocol were documented and reported } \\
\text { adequately, and did not indicate selective reporting }\end{array}$ \\
\hline Other bias & Low risk & $\begin{array}{l}\text { Quote from report: "Lupin Pharmaceuticals had no role in the conduct of the } \\
\text { trial, the analysis of the data, or the preparation of the manuscript" }\end{array}$ \\
\hline
\end{tabular}

Recruitment sites: Chennai, Madurai

Country where the trial was undertaken: India

Length of follow-up: 24 months after treatment completion

\section{Participants}

No. of participants randomized: 801 (of the 1650 anticipated)

Combined intervention arms: 3 to 4 months moxifloxacin: $N=629$

Control: 6 months ATT: $\mathrm{N}=172$

Age: $<35$ years: moxifloxacin $52 \%$; control $52 \%$

Gender: male: moxifloxacin $74 \%$; control $77 \%$

\section{Inclusion criteria:}

- Adult patients, 18 years or older, with newly diagnosed sputum smear positive pulmonary tuberculosis

- Resident within a designated study area and permitted home visits.

\section{Exclusion criteria:}

- Those with previous treatment for tuberculosis exceeding 30 days, weighing over $30 \mathrm{~kg}$, pregnant or lactating women

- Those with concomitant diabetes mellitus, severe systemic hypertension, epilepsy, serious forms of extrapulmonary tuberculosis, or HIV infection

Proportion with HIV seropositivity: nil (excluded)

Proportion with cavitation: moxifloxacin 36\%; control $41 \%$ 
Baseline drug resistance: isoniazid (moxifloxacin 7\%; control 8\%); ofloxacin (moxifloxacin 5\%; control $7 \%)$; rifampicin, ethambutol, isoniazid, and ethambutol; isoniazid and ofloxacin ( $<1 \%$ in both groups)

Interventions

Interventions: 3 - and 4-month moxifloxacin regimens: moxifloxacin added to standard ATT drugs

$\mathrm{N}=629$ randomized; 13 exclusions; 616 (98\%) evaluated of those randomized

- Rifampicin, isoniazid, pyrazinamide, ethambutol, and moxifloxacin daily for 3 months

- Rifampicin, isoniazid, pyrazinamide, ethambutol, and moxifloxacin daily for 2 months, followed by rifampicin, isoniazid, and moxifloxacin daily for 2 months

- Rifampicin, isoniazid, pyrazinamide, ethambutol, and moxifloxacin daily for 2 months, followed by rifampicin, isoniazid, and moxifloxacin thrice weekly for 2 months

- Rifampicin, isoniazid, pyrazinamide, ethambutol, and moxifloxacin daily for 2 months, followed by rifampicin, isoniazid, ethambutol, and moxifloxacin thrice weekly for 2 months

Control (6-month regimen): N = 172 randomized: 8 exclusions; 164 (95\%) evaluated of those randomized

- Rifampicin, isoniazid, pyrazinamide, and ethambutol thrice weekly for 2 months, followed by rifampicin and isoniazid thrice weekly for 4 months

Dosage: rifampicin 450 (<60 kg) or $600 \mathrm{mg}$ (>60 kg); isoniazid $300 \mathrm{mg}$ (daily) and $600 \mathrm{mg}$ (thrice weekly); pyrazinamide $1500 \mathrm{mg}$; ethambutol $800 \mathrm{mg}$ (daily) and $1200 \mathrm{mg}$ (thrice weekly); moxifloxacin 400 $\mathrm{mg}$

\section{Outcomes Outcomes reported and used in this review:}

- Sputum culture conversion at 2 months of treatment (assessed at 5 months)

- Adverse reactions while on anti-tuberculosis drugs

\section{Outcomes sought for the review but not reported:}

- Relapse rates 24 months after treatment among those with a favourable or doubtful bacteriologic response at end of treatment (primary outcome for the ongoing trial)

- Death from any cause

- Failure to complete treatment

- Treatment failure (bacteriologic response at end of treatment is an outcome in the ongoing trial)

- Acquired drug resistance

Follow-up method: over the 24-month follow-up period, participants had monthly clinical examination and adherence and adverse events recording; monthly sputum microscopy and culture (and on days 15 and 45) and monthly drug susceptibility testing for 1 positive culture; monthly ECG, haemogram, liver and kidney functions, random blood sugars, and HIV ELISA tests. Chest X-ray after the 2-month intensive phase. Adverse events were assessed monthly for the duration of treatment (3 to 6 months)

Treatment supervision: during the daily phase, treatment was under direct observation on 5 of 7 days of the week, whereas 2 doses were self-administered. All thrice-weekly phase doses were directly observed. Patients who missed treatment visits were visited at home and were motivated to attend the clinic for treatment

Trial registration ID: CTRI 2008/091/000024 (retrospectively registered on 09/05/2008)

Comment: results for sputum conversion at 2 months presented are the combined results of the 4 moxifloxacin regimens. Results for adverse events include up to the end of the 3- or 4-month moxifloxacin regimens and over 6 months in the control regimen 
Velayutham 2014 (Continued)

\begin{tabular}{lll} 
Bias & Authors' judgement & Support for judgement \\
\hline $\begin{array}{l}\text { Random sequence genera- } \\
\text { tion (selection bias) }\end{array}$ & Low risk & $\begin{array}{l}\text { Quote from report: "Restricted random allocation sequences generated using } \\
\text { random number tables,separately for the } 6 \text { strata, were used to assign the reg- } \\
\text { imens" }\end{array}$
\end{tabular}

\begin{tabular}{lll}
\hline $\begin{array}{l}\text { Allocation concealment } \\
\text { (selection bias) }\end{array}$ & Low risk & $\begin{array}{l}\text { Quote from trial registration document: "Sequentially numbered, sealed, } \\
\text { opaque envelopes" }\end{array}$ \\
\hline $\begin{array}{l}\text { Blinding of participants } \\
\begin{array}{l}\text { and personnel (perfor- } \\
\text { mance bias) }\end{array}\end{array}$ & Low risk & $\begin{array}{l}\text { This was an open-label study but all treatment arms had supervised treatment } \\
\text { and scheduled assessments for efficacy and safety outcomes }\end{array}$ \\
All outcomes &
\end{tabular}

\begin{tabular}{|c|c|c|}
\hline $\begin{array}{l}\text { Blinding of outcome as- } \\
\text { sessment (detection bias) } \\
\text { All outcomes }\end{array}$ & Low risk & $\begin{array}{l}\text { The outcomes reported are sputum culture results at } 2 \text { months and drug ad- } \\
\text { verse events that were assessed for all participants at specified time points. } \\
\text { Sputum culture results and ECG reports are objective outcomes, and the likeli- } \\
\text { hood of detection bias influencing the reporting of other adverse events is low }\end{array}$ \\
\hline
\end{tabular}

\begin{tabular}{|c|c|c|}
\hline $\begin{array}{l}\text { Incomplete outcome da- } \\
\text { ta (attrition bias): At the } \\
\text { end of ATT (Treatment fail- } \\
\text { ure, positive sputum cul- }\end{array}$ & Low risk & $\begin{array}{l}590 \text { of } 616(96 \%) \text { on moxifloxacin regimens and } 151 \text { of } 162(93 \%) \text { on the control } \\
\text { regimen had sputum cultures reported at } 2 \text { months. The } 3 \% \text { differential attri- } \\
\text { tion is unlikely to have influenced the difference in proportions with negative } \\
\text { sputum cultures at } 2 \text { months of } 14.6 \%(95 \% \mathrm{Cl} 8.8 \% \text { to } 21.8 \%)\end{array}$ \\
\hline
\end{tabular}

ture, treatment discontin-

uation, adverse events)

\begin{tabular}{|c|c|c|}
\hline $\begin{array}{l}\text { Incomplete outcome da- } \\
\text { ta (attrition bias): At the } \\
\text { end of follow-up (Relapse, } \\
\text { deaths) }\end{array}$ & Low risk & $\begin{array}{l}\text { Not reported, as the trial is ongoing and this report includes only interim out- } \\
\text { comes }\end{array}$ \\
\hline $\begin{array}{l}\text { Selective reporting (re- } \\
\text { porting bias) }\end{array}$ & Low risk & $\begin{array}{l}\text { This is an interim report of an ongoing trial. The outcomes presented were pre- } \\
\text { stated in the trial registration document }\end{array}$ \\
\hline Other bias & Low risk & No other sources of bias were detected \\
\hline
\end{tabular}

Abbreviations: ALT: alanine aminotransferase; ART: antiretroviral therapy; ARV: antiretroviral; AST: aspartate aminotransferase; ATT: anti-tuberculosis treatment; $\mathrm{CrCl}$ : creatinine clearance; DM: diabetes mellitus; DMID: Division of Microbiology and Infectious Disease; ECG: electrocardiogram; ELISA: enzyme-linked immunosorbent assay; IUCD: intrauterine contraceptive device; $M$ tuberculosis: Mycobacterium tuberculosis; MDR-TB: multi-drug-resistant tuberculosis; NIDDM: non-insulin-dependent diabetes mellitus; WHO: World Health Organization.

Characteristics of excluded studies [ordered by study ID]

\begin{tabular}{ll}
\hline Study & Reason for exclusion \\
\hline Alavi 2009 & $\begin{array}{l}\text { RCT comparing } 3 \text { months of rifampicin + isoniazid + ofloxacin versus a standard 6-month regimen } \\
\text { in people diagnosed with smear negative pulmonary tuberculosis }\end{array}$ \\
& Diagnosis of pulmonary tuberculosis was not confirmed by culture or GeneXpert \\
\hline Burman 2006 & $\begin{array}{l}\text { Factorial RCT comparing } 5 \text { days a week versus } 3 \text { days a week treatment with moxifloxacin substi- } \\
\text { tuting ethambutol in the intensive phase of treatment with isoniazid, rifampicin, and pyrazinamide } \\
\text { Not designed to compare treatments less than } 6 \text { months versus standard 6-month regimen (phase } \\
\text { 2b trial) }\end{array}$ \\
\hline
\end{tabular}




\begin{tabular}{ll}
\hline Study & Reason for exclusion \\
\hline Conde 2009 & $\begin{array}{l}\text { RCT comparing moxifloxacin versus ethambutol in the intensive phase of treatment with ri- } \\
\text { fampicin, isoniazid, and pyrazinamide }\end{array}$ \\
$\begin{array}{l}\text { Not designed to compare treatments less than } 6 \text { months versus standard 6-month regimen (phase } \\
\text { 2b trial) }\end{array}$
\end{tabular}

Conde 2016 RCT comparing rifapentine plus moxifloxacin or rifampin plus ethambutol daily for 8 weeks, along with isoniazid and pyrazinamide

Not designed to compare treatments less than 6 months versus standard 6-month regimen (phase $2 b$ trial)

Dorman 2009

RCT comparing moxifloxacin versus isoniazid in the intensive phase of treatment with rifampicin, ethambutol, and pyrazinamide

Not designed to compare treatments less than 6 months versus standard 6-month regimen (phase $2 \mathrm{~b}$ trial)

\section{El-Sadr 1998}

RCT comparing levofloxacin added for the first 2 months to the standard 6-month ATT regimen versus 6 to 9 months of standard ATT regimen

No comparison with a regimen shorter than 6 months

Johnson $2009 \begin{aligned} & \text { RCT in adults with newly diagnosed, sputum- or culture-confirmed, non-cavitary pulmonary tu- } \\ & \text { berculosis who were culture negative after } 4 \text { months of daily treatment with } 2 \mathrm{HRZE}+2 \mathrm{HR} \text { to stop } \\ & \text { treatment (4-month treatment arm) or continue HR for } 2 \text { months }\end{aligned}$
$\begin{aligned} & \text { Participants had already taken } 4 \text { months of ATT before randomization. Only those who were spu- } \\ & \text { tum-negative were randomized, After randomization, participants received either no treatment or } \\ & \text { only } 2 \text { more months of HR }\end{aligned}$

Kohno 1992

Contolled trial comparing ofloxacin, rifampicin, and isoniazid with the regimen of ethambutol, rifampicin, and isoniazid given daily for 9 months

No control arm with 2HRZE + 4HR (or 4HRE) or treatment arm with shorter regimens

RCT (phase 2) comparing three 6-month regimens with gatifloxacin, moxifloxacin, or ofloxacin given along with rifampicin, isoniazid, and pyrazinamide for the first 2 months followed by 4 months of isoniazid and rifampicin versus standard $2 \mathrm{HRZE}+4 \mathrm{HR}$ regimens

No arm with shorter regimens

\begin{tabular}{ll}
\hline $\begin{array}{l}\text { Tuberculosis Research Centre } \\
1986\end{array}$ & $\begin{array}{l}\text { Controlled trial comparing } 3 \text { and } 5 \text { months of streptomycin, isoniazid, and pyrazinamide, with or } \\
\text { without rifampicin }\end{array}$
\end{tabular}

No control arm with $2 \mathrm{HRZE}+4 \mathrm{HR}$ (or 4HRE) Tuberculosis Research Centre RCT comparing 4 different regimens of ofloxacin, isoniazid, rifampicin, and pyrazinamide
2002
No control arm with $2 \mathrm{HRZE}+4 \mathrm{HR}$ (or 4HRE)

Abbreviations: ATT: anti-tuberculosis treatment; E: ethambutol; H: isoniazid; R: rifampicin; RCT: randomized controlled trial; Z: pyrazinamide.

\section{Characteristics of ongoing studies [ordered by study ID]}


CTRI/2008/091/000024

Trial name or title

Randomized clinical trial to study the efficacy and tolerability of 3- and 4-month regimens containing moxifloxacin in the treatment of patients with sputum smear and culture positive pulmonary tuberculosis

\begin{tabular}{ll}
\hline Methods & Randomized, open-label, parallel-group, 5-arm, active-controlled trial \\
\hline Participants & Inclusion criteria: \\
- Age 18 years to 60 years \\
- No previous anti-tuberculosis treatment \\
- At least 2 sputum smears should be positive for tubercle bacilli by fluorescent mic \\
- - Willing to attend the treatment centre for supervised treatment \\
- Willing for home visits by staff from the centre \\
- Willing to give written informed consent \\
Exclusion criteria: \\
- Body weight less than 30 kg \\
- Hepatic or renal disease as evidenced by clinical or biochemical abnormalities \\
- Diabetes mellitus \\
- History of seizure or loss of consciousness \\
- Psychiatric illness \\
- Thormal electrocardiogram or anti-arrhythmic medication \\
- Seropositive for HIV antibodies \\
- Pregnancy or lactation \\
Visual disorders other than refractory error
\end{tabular}

Anticipated sample size: 1650

\section{Intervention(s):}

Moxifloxacin arm: 4 regimens of 3 to 4 months

\section{RHZEM}

\section{RHZEM/2 RHM}

2 RHZEM/2 RHM thrice weekly

2 RHZEM/2 RHEM thrice weekly

Dose: rifampicin $450 \mathrm{mg}$; isoniazid $300 \mathrm{mg}$ (daily), $600 \mathrm{mg}$ (thrice weekly); pyrazinamide $1500 \mathrm{mg}$; ethambutol $800 \mathrm{mg}$ (daily), $1200 \mathrm{mg}$ (thrice weekly); moxifloxacin $400 \mathrm{mg}$

\section{Control:}

2 RHZE thrice weekly/4 RH thrice weekly (for 6 months)

Dose: rifampicin $450 \mathrm{mg}$; isoniazid $600 \mathrm{mg}$; pyrazinamide $1500 \mathrm{mg}$; ethambutol $1200 \mathrm{mg}$

Primary outcome:

- Relapse rates 24 months after treatment among those with a favourable or doubtful bacteriologic response at end of treatment

\section{Secondary outcomes:}


CTRI/2008/091/000024 (Continued)

- Sputum culture conversion at 2 months of treatment

- Bacteriologic response at end of treatment

- Adverse reactions to anti-tuberculosis drugs during treatment

Starting date

Contact information

Notes
30 May 2007; anticipated study end date: May 2015; no results posted (last modified 06/02/2013)

Dr MS Jawahar, Tuberculosis Research Centre, Mayor VR Ramanathan Road, Chetput, Chennai TAMIL NADU 600031 India. Tel: +91-44-28369500; Email: msjawahar@trcchennai.in

Study locations: Chennai and Madurai in India

Registration number: CTRI/2008/091/000024

Primary sponsor: Indican Council of Medical Research

Comment: the first author confirmed that this is completed, has been analysed, written up, submitted for publication, and will be re-submitted after peer review

\section{ISRCTN63579542}

Trial name or title

Methods

Shorter treatment for minimal tuberculosis in children (SHINE study)

Parallel-group, randomized, non-inferiority, open-label, 2-arm, phase 3 clinical trial

\section{Participants}

\section{Inclusion criteria:}

- Age 0 to 16 years

- Weight $>4 \mathrm{~kg}$

- Clinician has decided to treat with standard first-line regimen

- Asymptomatic or symptomatic but with non-severe tuberculosis including not previously treated for tuberculosis or successfully treated for tuberculosis over 2 years since last completed treatment

- Known HIV status: HIV infected or HIV uninfected

- Willing and likely to adhere to 72 weeks of follow-up

- Informed written consent from parent/legal caregiver

- Home address accessible for visiting and intending to remain within recruitment area for follow-up

\section{Exclusion criteria:}

- Smear positive respiratory sample tuberculosis

- Premature (<37 weeks) and aged under 3 months

- Miliary tuberculosis, spinal tuberculosis, tuberculosis meningitis, osteoarticular tuberculosis, abdominal tuberculosis, congenital tuberculosis

- Pre-existing liver or kidney disease, peripheral neuropathy, cavitation

- Any known contraindication to taking anti-tuberculosis drugs

- Known contact with MDR, pre-XDR, or XDR adult source case

- Proven anti-tuberculosis drug resistance in the child

- Severely sick

- Pregnancy

Anticipated sample size: 1200 
ISRCTN63579542 (Continued)

8 weeks intensive Isoniazid $(H)$, rifampicin $(R)$, pyrazinamide $(Z)$ with or without ethambutol $(E)$ according to local practice, HRZ(E), followed by

8 weeks of continuation HR

Control: 6-month standard ATT regimen

8 weeks intensive HRZ(E), followed by

6 weeks of continuation HR

\section{Outcomes}

\section{Primary outcome measures:}

- Efficacy: unfavourable outcome, defined by the composite endpoint of tuberculosis treatment failure, relapse (or re-infection), or death

- Safety: grade $3 / 4$ adverse events

\section{Secondary outcome measures:}

- Mortality

- Adverse drug reactions up to 30 days of completing treatment

- Unfavourable outcome in those with definite tuberculosis

- Suppressed HIV viral load at 24 and 48 weeks in HIV-infected children starting ART, measured centrally on stored samples

- Adherence and acceptability

- Bacterial infection

Anciliary studies will evaluate pharmacokinetics; cost/cost-effectiveness implications of treatment shortening, and a nested qualitative substudy will investigate the ways in which health workers manage implementation of dose and weight band recommendations, particularly in children taking anti-tuberculosis drugs and ARVs

\begin{tabular}{ll}
\hline Starting date & April 2015; anticipated end date: April 2019 (no longer recruiting) \\
\hline Contact information & SHINE Trial Management Team \\
MRC Clinical Trials Unit at UCL \\
Institute of Clinical Trials and Methodology \\
Aviation House, 125 Kingsway, London WC2B 6NH, UK \\
Ph: +44 (0) 20 7670 4700 \\
Email: SHINE.MRCCTU@ucl.ac.uk
\end{tabular}

Notes

Study locations: South Africa, India, Uganda, Zambia

Registration number: ISRCTN63579542

Primary sponsors: University College London, Joint Global Health Trials Scheme: Department for International Development, the Wellcome Trust, the Medical Research Council, and Svizera Ltd

\section{NCT02342886}

Trial name or title

Shortening treatment by advancing novel drugs (STAND)

Shortened treatment regimens versus the standard regimen for drug-sensitive pulmonary tuberculosis (Review) 
NCT02342886 (Continued)

Methods
Phase 3 open-label, partially randomized, controlled clinical trial
Participants

\section{Inclusion criteria:}

- Signed written consent or witnessed oral consent in the case of illiteracy, before undertaking any trial-related procedures

- Male or female, aged 18 years or older

- Body weight (in light clothing and no shoes) $\geq 30 \mathrm{~kg}$

- Sputum positive for tubercle bacilli (at least $1+$ on the International Union Against Tuberculosis and Lung Disease (IUATLD) and World Health Organization (WHO) scales on smear microscopy at the trial laboratory)

- Drug-sensitive tuberculosis treatment arm participants should be:sensitive to rifampicin by rapid sputum-based test (may be sensitive or resistant to isoniazid) AND either newly diagnosed with TB or with patient history of being untreated for at least 3 years after cure from a previous episode of TB. If they are entered into the trial because they are sensitive to rifampicin by rapid sputum-based test, however, if receipt of rifampicin resistance testing via an indirect susceptibility test in liquid culture shows they are rifampicin resistant, they will be. excluded as late exclusions; possibly replaced as determined by the sponsor

- MDR-TB treatment arm participants should be resistant to rifampicin by rapid sputum-based test (may be sensitive or resistant to isoniazid)

- Chest X-ray that in the opinion of the investigator is compatible with pulmonary TB

- Non-childbearing potential or using effective methods of birth control, as defined below

Non-childbearing potential:

- Participant not heterosexually active or practicing sexual abstinence; or

- Female participant or male participant with female sexual partner - bilateral oophorectomy, bilateral tubal ligation, and/or hysterectomy; or postmenopausal with a history of no menses for at least 12 consecutive months; or

- Male participant or female participant with male sexual partner - vasectomized or with bilateral orchidectomy minimally 3 months before screening

Effective birth control methods:

- Double-barrier method, which can include male condom, diaphragm, cervical cap, or female condom; or

- Female participant: barrier method combined with hormone-based contraceptives or an intrauterine device for the female participant

- Male participant's female sexual partner: double-barrier method or hormone-based contraceptives or an intrauterine device for the female participant

Willing to continue practising birth control methods and not planning to conceive throughout treatment and for 12 weeks (male participants) or 1 week (female participants) after last dose of trial medication or discontinuation from trial medication in case of premature discontinuation

\section{Exclusion criteria:}

- Any non-TB-related condition (including myasthenia gravis) where participation in the trial, as judged by the investigator, could compromise the well-being of the participant or could prevent, limit, or confound protocol-specified assessments

- Being or about to be treated for malaria

- Critically ill and, in the judgement of the investigator, with a diagnosis likely to result in death during the trial or the follow-up period

- TB meningitis or other forms of extrapulmonary tuberculosis with high risk of a poor outcome, or likely to require a longer course of therapy (such as TB of the bone or joint), as judged by the investigator

- History of allergy or hypersensitivity to any of the trial IMP or related substances, including known allergy to any fluoroquinolone antibiotic, history of tendinopathy associated with quinolones, or suspected hypersensitivity to any rifampicin antibiotics 
- For HIV-infected participants, any of the following: CD4+ count $<100$ cells/ $\mu \mathrm{L}$; Karnofsky score < $60 \%$; received intravenous antifungal medication within the last 90 days; WHO clinical stage 4 HIV disease

- Resistant to fluoroquinolones (rapid, sputum-based molecular screening tests). If they are entered into the trial because they are sensitive to fluoroquinolones by rapid sputum-based test, but on receipt of the fluoroquinolone resistance test via an indirect susceptibility test in liquid culture, they show they are fluoroquinolone resistant, they will be excluded as late exclusions; possibly replaced as determined by the sponsor

- Resistant to pyrazinamide (rapid, sputum-based molecular screening tests). Drug-sensitive TB treatment arm participants may be entered before receipt of the rapid, sputum-based molecular pyrazinamide resistance screening test result. On receipt of the result, if resistant, they will be excluded as late exclusions; possibly replaced as determined by the sponsor. MDR-TB treatment arm participants may not be entered before receipt of the rapid, sputum-based molecular pyrazinamide resistance screening test result showing they are sensitive to pyrazinamide

- Having participated in other clinical trials with investigational agents within 8 weeks before trial start or currently enrolled in an investigational trial

- With any of the following at screening (per measurements and reading done by central electrocardiogram (ECG) where applicable): cardiac arrhythmia requiring medication; prolongation of QT/ QTc interval with QTcF (Fridericia correction) $>450 \mathrm{~ms}$; history of additional risk factors for torsade de pointes (e.g. heart failure, hypokalaemia, family history of long QT syndrome); any clinically significant ECG abnormality, in the opinion of the investigator

- Unstable diabetes mellitus that required hospitalization for hyperglycaemia or hypoglycaemia within the past year before the start of screening. Specific treatments:

* Previous treatment with PA-824 as part of a clinical trial

* For DS-TB treatment arms: previous treatment for tuberculosis within 3 years before Day (-9 to -1) (screening). Participants who have previously received isoniazid prophylactically may be included in the trial as long as that treatment is/was discontinued at least 7 days before randomization into this trial. For MDR-TB participants: previous treatment for MDR-TB, although may have been on MDR-TB treatment regimen for no longer than 7 days at the start of screening. Previous treatment for TB includes, but is not limited to, gatifloxacin, amikacin, cycloserine, rifabutin, kanamycin, para-aminosalicylic acid, rifapentine, thiacetazone, capreomycin, quinolones, thioamides, and metronidazole

* Any disease or condition for which use of standard TB drugs or any of their components is contraindicated, including but not limited to allergy to any TB drugs, their components, or the IMP

* Use of any drug within 30 days before randomization known to prolong QTc interval (including, but not limited to, amiodarone, amitriptyline, bepridil, chloroquine, chlorpromazine, cisapride, clarithromycin, disopyramide dofetilide, domperidone, droperidol, erythromycin, halofantrine, haloperidol, ibutilide, levomethadyl, mesoridazine, methadone, pentamidine, pimozide, procainamide, quinacrine, quinidine, sotalol, sparfloxacin, thioridazine)

* Use of systemic glucocorticoids within 1 year of start of screening (inhaled or intranasal glucocorticoids are allowed)

* Participants recently started or expected to need to start antiretroviral therapy (ART) within 1 month after randomization. Patients may be included who have been on ARTs for longer than 30 days before the start of screening, or who are expected to start ART more than 30 days after randomization. Laboratory abnormalities 
NCT02342886 (Continued)

- Participants with the following toxicities at screening as defined by the enhanced Division of Microbiology and Infectious Disease (DMID) adult toxicity table (November 2007), where applicable:

* Creatinine grade 2 or greater (> 1.5 times upper limit of normal (ULN))

* Creatinine clearance $(\mathrm{CrCl})$ level less than $30 \mathrm{~mL} / \mathrm{min}$ according to the Cockcroft-Gault formula

* Haemoglobin grade $4\left(<6.5 \mathrm{~g} / \mathrm{dL}\right.$ ); platelets grade 3 or greater (under $50 \times 10^{9}$ cells/L 50,000/ $\mathrm{mm}^{3}$ )

* Serum potassium less than lower limit of normal for the laboratory; this may be repeated once

* Aspartate aminotransferase (AST) grade 3 or greater ( $\geq 3.0 \times$ ULN)

* Alanine aminotransferase (ALT) grade 3 or greater ( $\geq 3.0 \times$ ULN)

* Alkaline phosphatase (ALP): grade 4 (>8.0 $\times$ ULN) to be excluded; grade 3 ( $\geq 3.0$ to $8.0 \times$ ULN) must be discussed with and approved by the sponsor medical monitor

* Total bilirubin: $2.0 \times$ ULN, when other liver functions are in the normal range; $1.50 \times$ ULN when accompanied by any increase in other liver function tests among participants with total bilirubin $>1.25 \times$ ULN and accompanied by any increase in other liver function tests must be discussed with the sponsor medical monitor before enrolment

Recruited sample size $=1500$

Interventions

Interventions:

- Moxifloxacin $400 \mathrm{mg}+$ PA-824 $200 \mathrm{mg}$ + pyrazinamide $1500 \mathrm{mg}$ orally once daily for 26 weeks

- Moxifloxacin $400 \mathrm{mg}+\mathrm{PA}-824200 \mathrm{mg}+$ pyrazinamide $1500 \mathrm{mg}$ orally once a day for 17 weeks

- Moxifloxacin $400 \mathrm{mg}+$ PA-824 $100 \mathrm{mg}$ + pyrazinamide $1500 \mathrm{mg}$ orally once daily for 17 weeks

\section{Control:}

- $2 \mathrm{HRZE} / 4 \mathrm{HR}$ (26 weeks)

(Additional MDR-TB arm: moxifloxacin 400 mg + PA-824 200 mg + pyrazinamide 1500 mg for 26 weeks)

\section{Primary outcome:}

- Incidence of combined bacteriologic failure or relapse of clinical failure at 12 months from start of therapy

* Bacteriologic failure: during the treatment period, failure to attain culture conversion to negative status in liquid culture

* Bacteriologic relapse: during the follow-up period, failure to maintain culture conversion to negative status in liquid culture, with culture conversion to positive status with a Mycobacterium tuberculosis (MTB) strain that is genetically identical to the infecting strain at baseline

* Bacteriologic reinfection: during the follow-up period, failure to maintain culture conversion to negative status in liquid culture, with culture conversion to positive status with MTB strain that is genetically different from the infecting strain at baseline

* Clinical failure: change from protocol-specified TB treatment due to treatment failure, re-treatment for TB during follow-up, or TB-related death

\section{Secondary outcomes:}

- Incidence of bacteriologic failure or relapse or clinical failure at 24 months from the start of therapy as a confirmatory analysis

- Rate of change in time to culture positivity (TTP) over time in liquid culture MGIT in sputum, represented by the model-fitted $\log ($ TTP) results as calculated by the regression of the observed $\log ($ TTP) results over time. [Screening; Day 1, 7; Week 2 to 7 ; Month 2 to $6,9,12,15,18,24$ ] MGIT is defined as mycobacterial growth indicator tube

- Time in days to sputum culture conversion to negative status in liquid culture (MGIT) through the treatment period to be explored as a potential biomarker of definitive outcome. [Screening; Day $1,7$; Week 2 to 7 ; Month 2 to $6,9,12,15,18,24]$ 
- Proportion of participants with sputum culture conversion to negative status in liquid culture (MGIT) at 4, 8, 12, and 17 weeks to be explored as a potential biomarker of definitive outcome. [Week 4, 8, 12, and 17]

- Incidence of treatment-emergent adverse events (TEAEs) presented by incidence and seriousness, leading to TB-related or non-TB-related death. [Day 1, 7; Week 2 to 7; Month 2 to 6, 9, 12, $15,18,24]$

- Clinical laboratory safety measurements of haematology and chemistry, including observed and change from baseline. [Screening; Day 1; Week 1, 2, 4; Month 2, 3, 4, 6]

- Trough plasma concentrations will be used to evaluate effects of baseline subject covariates on trial drug pharmacokinetics and associated bacteriologic endpoints. [Week 2, Month 2]

- Minimum inhibitory concentration (MIC) against moxifloxacin and PA-824 [Day 1; Week 17 or Week 26 ]. MIC: lowest concentration of moxifloxacin or PA-824 that will inhibit visible growth in culture

- Change from baseline in sperm concentration by group. [Screening; Day 1; Week 12, 13, 26, 27, 39, 40]

- Change from baseline in male FSH by group. [Screening; Day 1; Week 12, 13, 26, 27, 39, 40] Reproductive hormones: $\mathrm{FSH}$, LH, testosterone, inhibin B

- Change from baseline in male LH by group. [Screening; Day 1; Week 12, 13, 26, 27, 39, 40]

- Change from baseline in male testosterone by group. [Screening; Day 1; Week 12, 13, 26, 27, 39, 40]

- Change from baseline in male inhibin B by group. [Screening; Day 1; Week 12, 13, 26, 27, 39, 40]

- Change from baseline in proportion of total motile sperm by group. [Screening; Day 1; Week 12, $13,26,27,39,40]$

- Change from baseline in sperm morphology by group. [Screening; Day 1; Week 12, 13, 26, 27, 39, 40]

- Change from baseline in sperm volume by group. [Screening; Day 1; Week 12, 13, 26, 27, 39, 40]

- Change from baseline in total sperm numbers by group. [Screening; Day 1; Week 12, 13, 26, 27, $39,40]$

Email: shg3@st-andrews.ac.uk

Sponsors: Global Alliance for TB Drug Development

\section{NCT02410772}

\begin{tabular}{ll}
\hline Trial name or title & Rifapentine-containing tuberculosis treatment shortening regimens \\
\hline Methods & Randomized, open-label, parallel-assignment, controlled phase 3 clinical trial \\
\hline Participants & Inclusion criteria: \\
- Suspected pulmonary tuberculosis plus 1 or both of the following: (a) at least 1 sputum specimen \\
positive for acid-fast bacilli on smear microscopy OR (b) at least 1 sputum specimen positive for \\
M tuberculosis by Xpert MTB/RIF testing, with semi-quantitative result of 'medium' or 'high' and \\
rifamycin resistance not detected \\
- Age 12 years or older \\
Verifiable address or residence location that is readily accessible for visiting, and willingness to \\
inform the study team of any change in address during treatment and follow-up period
\end{tabular}


- Women of childbearing potential who are not surgically sterilized must agree to practice a barrier method of contraception or must abstain from heterosexual intercourse during study drug treatment

- Documentation of HIV infection status

- For HIV-positive individuals, CD4 T-cell count greater than or equal to 100 cells $/ \mathrm{mm}^{3}$ based on testing performed at or within 30 days before screening

- Laboratory parameters done at or within 14 days before screening:

* Serum or plasma alanine aminotransferase (ALT) less than or equal to 3 times upper limit of normal

* Serum or plasma total bilirubin less than or equal to 2.5 times upper limit of normal

* Serum or plasma creatinine level less than or equal to 2 times upper limit of normal

* Serum or plasma potassium level greater than or equal to $3.5 \mathrm{meq} / \mathrm{L}$

* Hemoglobin level $7.0 \mathrm{~g} / \mathrm{dL}$ or greater

* Platelet count $100,000 / \mathrm{mm}^{3}$ or greater

- For women of childbearing potential, a negative pregnancy test at or within seven (7) days before screening:

* Karnofsky score greater than or equal to 60

* Written informed consent

\section{Exclusion criteria:}

- Pregnant or breastfeeding

- Unable to take oral medications

- Previously enrolled in this study

- Received any investigational drug in the past 3 months

- More than five (5) days of treatment directed against active tuberculosis within 6 months preceding initiation of study drugs

- More than five (5) days of systemic treatment with any 1 or more of the following drugs within 30 days preceding initiation of study drugs: isoniazid, rifampin, rifabutin, rifapentine, ethambutol, pyrazinamide, kanamycin, amikacin, streptomycin, capreomycin, moxifloxacin, levofloxacin, gatifloxacin, ofloxacin, ciprofloxacin, other fluoroquinolones, ethionamide, prothionamide, cycloserine, terizidone, para-aminosalicylic acid, linezolid, clofazimine, delamanid, or bedaquiline

- Known history of prolonged QT syndrome

- Suspected or documented tuberculosis involving central nervous system and/or bones and/or joints, and/or miliary tuberculosis, and/or pericardial tuberculosis

- Current or planned use within 6 months following enrolment of 1 or more of the following medications: HIV protease inhibitors, HIV integrase inhibitors, HIV entry and fusion inhibitors, HIV nonnucleoside reverse transcriptase inhibitors other than efavirenz, quinidine, procainamide, amiodarone, sotalol, disopyramide, ziprasidone, or terfenadine. Individuals who are currently taking efavirenz-based antiretroviral treatment (ART) or for whom initiation of efavirenz-based ART is planned within 17 weeks following enrolment may participate

- Weight less than $40.0 \mathrm{~kg}$

- Known allergy or intolerance to any of the study medications

- Individuals will be excluded from enrolment if, at the time of enrolment, their $M$ tuberculosis isolate is already known to be resistant to any 1 or more of the following: rifampin, isoniazid, pyrazinamide, ethambutol, or fluoroquinolones

- Other medical conditions that, in the investigator's judgment, make study participation not in the individual's best interest

- Current or planned incarceration or other involuntary detention

Anticipated sample size: 2500

\section{Standard ATT drugs: 4-month (17 weeks) regimen}

8 weeks of daily treatment with rifapentine, isoniazid, pyrazinamide, and ethambutol, followed by 
NCT02410772 (Continued)
9 weeks of daily treatment with rifapentine and isoniazid

Moxifloxin combination: 4-month (17 weeks) regimen: moxifloxacin for 4 months substituting ethambutol in intensive phase

8 weeks of daily treatment with rifapentine, isoniazid, pyrazinamide, and moxifloxacin, followed by

9 weeks of daily treatment with rifapentine, isoniazid, and moxifloxacin

\section{Control: standard 6-month (26-week) ATT regimen}

8 weeks of daily treatment with rifampin, isoniazid, pyrazinamide, and ethambutol, followed by

18 weeks of daily treatment with rifampin and isoniazid

\section{Dosing:}

All drugs are administered orally, 7 days/week, directly observed by a healthcare worker at least 5 of the 7 days each week. Pyridoxine (vitamin B6), 25 or $50 \mathrm{mg}$, is administered with each study dose

Study drug doses: rifampin $600 \mathrm{mg}$; isoniazid $300 \mathrm{mg}$; pyrazinamide < $55 \mathrm{~kg} 1000 \mathrm{mg}, \geq 55$ to $75 \mathrm{~kg}$ $1500 \mathrm{mg},>75 \mathrm{~kg} 2000 \mathrm{mg}$; ethambutol < $55 \mathrm{~kg} 800 \mathrm{mg}, \geq 55$ to $75 \mathrm{~kg} 1200 \mathrm{mg},>75 \mathrm{~kg} 1600 \mathrm{mg}$

\section{Primary outcome measures:}

- TB disease-free survival at 12 months after study treatment assignment [Time Frame: 12 months after treatment assignment]

- Proportion of participants with grade 3 or higher adverse events during study drug treatment [Time Frame: 4 or 6 months]

\section{Secondary outcome measures:}

- TB disease-free survival at 18 months after study treatment assignment [Time Frame: 18 months after treatment assignment]

- Proportion of participants who are culture negative at 8 weeks [Time Frame: 8 weeks]

Solid and liquid media considered separately:

- Time to stable sputum culture conversion [Time Frame: 4 or 6 months] - solid and liquid media considered separately

- Speed of decline of sputum viable bacilli by automated MGIT days to detection [Time Frame: 4 or 6 months]

- TB disease-free survival at 12 and 18 months after study treatment assignment, assuming all losses to follow-up and non-tuberculosis deaths have an unfavourable outcome [Time Frame: 18 months after study treatment assignment]. Sensitivity analyses, assuming all losses to follow-up and non-tuberculosis deaths have an unfavourable outcome

- TB disease-free survival at 12 and 18 months after study treatment assignment, assuming all losses to follow-up and non-tuberculosis deaths have a favourable outcome [Time Frame: 18 months after study treatment assignment]. Sensitivity analyses, assuming all losses to follow-up and nontuberculosis deaths have a favourable outcome

- Discontinuation of assigned treatment for a reason other than microbiological ineligibility [Time Frame: 4 or 6 months]

- Efavirenz maximum concentration, area under the time-concentration curve, and half-life [Time Frame: 4 months]

Among participants with HIV infection receiving efavirenz-based antiretroviral therapy, these values will be used to estimate steady state efavirenz PK parameters including mid-dosing interval concentration 
NCT02410772 (Continued)

Contact information Stefan Goldberg, Centers for Disease Control and Prevention. Ph: 404-639-5339; Email: ssg3@cdc.gov
Study locations: 38 including Brazil, China, India, and Malawi

Registration number: NCT02410772

Sponsor: Centers for Disease Control and Prevention

Collaborator: AIDS Clinical Trials Group
Trial name or title

Methods

Participants
An international multi-centre controlled clinical trial to evaluate $1200 \mathrm{mg}$ and $1800 \mathrm{mg}$ rifampicin daily in the reduction of treatment duration for pulmonary tuberculosis from 6 months to 4 months (RIFASHORT)

\section{Inclusion criteria:}

- Patients with:

* Newly diagnosed, smear microscopy positive, pulmonary tuberculosis, rifampicin-susceptible MTBC confirmed by Xpert MTB/RIF OR

* Smear microscopy negative, suspected pulmonary tuberculosis, confirmed by Xpert MTB/RIF as sputum MTBC positive and rifampicin susceptible

- No more than 1 week of previous treatment for tuberculosis, for active TB or confirmed or presumed latent TB infection

- $\geq 18$ years old

- Consent to participation in the trial and to HIV testing

- Provide informed consent

- Stable home address within easy reach of the treatment facility and likely to remain there for the entire treatment and follow-up period

- Women who are pre-menopausal or of childbearing age must be using a barrier form of contraception (condoms, diaphragms, cervical caps, or contraceptive sponges), or must be surgically sterilized or have an intrauterine coil device (IUCD) in place for the duration of the treatment phase; alternatively they should agree to abstain from sexual activity during the treatment phase.

\section{Exclusion criteria:}

- Rifampicin resistance identified by Xpert MTB/RIF or by direct susceptibility testing (late exclusions)

- Moribund phase

- TB meningitis or extrapulmonary TB

- Female and known to be pregnant or breastfeeding

- Condition likely to lead to uncooperative behaviour such as psychiatric illness or alcoholism

- History of seizures

- Contraindications to any medications in the study regimens

- HIV positive according to local testing algorithm

- Blood disorder (including grade 4 or above thrombocytopenia)

- Haemoglobin $<7 \mathrm{~g} / \mathrm{dL}$

- Peripheral neuritis

- Pre-existing liver disease

- ALT > 5 times upper limit of normal (ULN) for that laboratory

- Raised bilirubin (grade 4 or above) 
NCT02581527 (Continued)

- Kidney disease

- Estimated creatinine clearance $<30 \mathrm{~mL} / \mathrm{min}$

- Previously diagnosed diabetes mellitus (non-insulin-dependent or insulin-dependent)

- $\mathrm{HbA} 1 \mathrm{c}>48 \mathrm{mmol} / \mathrm{mol}$

- Weight $<35 \mathrm{~kg}$

- Taking any of the excluded medications listed in the Summary of Product Characteristics (SmPC) for any trial drugs

- Pre-existing non-tuberculous disease likely to prejudice response to, or assessment of, treatment as judged by the Principal Investigator

Anticipated sample size: 654

Interventions

\section{Interventions: 4-month regimens}

Rifampicin 1200 mg combination ATT regimen

2 months of daily ethambutol, isoniazid, rifampicin, and pyrazinamide, followed by

2 months of daily isoniazid and rifampicin

Supplement of $450 \mathrm{mg}$ (weight bands 35 to $39 \mathrm{~kg}$ and 40 to $54 \mathrm{~kg}$ ) or $600 \mathrm{mg}$ (weight band 55 to 69 $\mathrm{kg}$ and 70 and more $\mathrm{kg}$ ) of rifampicin will be given throughout the 4 months (2EHR 1200Z/2HR1200)

\section{Rifampicin 1800 mg combination ATT regimen}

2 months of daily ethambutol, isoniazid, rifampicin, and pyrazinamide, followed by

2 months of daily isoniazid and rifampicin

Supplement of $450 \mathrm{mg}$ (weight bands 35 to $39 \mathrm{~kg}$ and 40 to $54 \mathrm{~kg}$ ) or $600 \mathrm{mg}$ (weight band 55 to 69 $\mathrm{kg}$ and 70 and more $\mathrm{kg}$ ) of rifampicin will be given throughout the 4 months (2EHR1800Z/2HR1800)

\section{Control:}

Standard 6-month ATT regimen

2 months of the standard regimen of isoniazid, pyrazinamide, and ethambutol plus $10 \mathrm{mg} / \mathrm{kg}$ rifampicin for the initial 8 weeks, followed by

4 months of isoniazid and rifampicin (at the same dose size) for an additional 4 months (2HRZE/4HR).
Outcomes

Starting date

\section{Primary outcomes:}

- Efficacy: proportion with a combined unfavourable endpoint measured 18 months from randomization; this end point includes failure at end of treatment, recurrence, and death. This will be measured in the modified intent-to-treat microscopy positive population

- Safety: occurrence of grade 3 or 4 adverse events at any time during chemotherapy and 1 month post therapy in the intent-to-treat population with an MTBC positive test result Xpert MTB/RIF positive population

\section{Secondary outcomes}

- Per-protocol analysis of the primary efficacy outcome

- Combined unfavourable endpoint measured 18 months from randomization in the Xpert MTB/RIF positive (i) modified intent-to-treat and (ii) per-protocol populations

- Sputum cultures positive for $M$ tuberculosis at 8 and 12 weeks from randomization

- Any adverse event, up to 1 month after end of treatment, graded according to DAIDS criteria

- Time to unfavourable outcome in the modified to intent-to-treat and per-protocol microscopy positive population 
NCT02581527 (Continued)

Contact information

Notes
Eduardo Rómulo Chávez Ticona. Calle Rio Huaura Nro. 319, Pueblo Libre, Lima, Peru Pueblo Libre Lima LIMA Perú. Ph: 993560268; Email: eticonacrg@gmail.com

Study locations: Bolivia, Botswana, Peru, Uganda

Registration number: NCT02581527

Primary sponsor: St Georges University of London

\begin{tabular}{|c|c|}
\hline Trial name or title & Shortened regimens for drug-susceptible pulmonary tuberculosis \\
\hline Methods & Multi-centre, randomized, non-inferiority, open-label, controlled phase 3 clinical trial \\
\hline \multirow[t]{11}{*}{ Participants } & $\begin{array}{l}\text { Patients with newly diagnosed drug-susceptible pulmonary TB who fulfil the inclusion and exclu- } \\
\text { sion criteria }\end{array}$ \\
\hline & Inclusion criteria: \\
\hline & - Willing and able to give informed consent to participate in trial treatment and follow-up \\
\hline & - Aged 18 to 65 years \\
\hline & $\begin{array}{l}\text { - Twice positive acid-fast bacilli (AFB) sputum smear or positive sputum culture result, along with } \\
\text { chest X-ray consistent with active pulmonary tuberculosis }\end{array}$ \\
\hline & - Newly diagnosed cases receiving anti-tuberculosis treatment for $<1$ month \\
\hline & $\begin{array}{l}\text { - Urine human chorionic gonadotropin (U-HCG) negative and must agree to use effective contra- } \\
\text { ception during trial period }\end{array}$ \\
\hline & - Alanine aminotransferase (ALT) and total bilirubin $<2$ times upper limit of normal \\
\hline & - Creatinine clearance rate $>30 \mathrm{~mL} / \mathrm{min}$ \\
\hline & - Haemoglobin $>7.0 \mathrm{~g} / \mathrm{dL}$ \\
\hline & - Platelets $>50 \times 10^{9} / \mathrm{L}$ \\
\hline
\end{tabular}

\section{Exclusion criteria:}

- Concomitant severe cardiovascular, liver, kidney, nervous system, haematopoietic system, and other disease, or concomitant neoplastic disease. Or extensive lesion with respiratory insufficiency

- Uncontrolled diabetes mellitus

- Concomitant mental disorder

- HIV-positive individuals

- Critically ill patients

- Pregnant or breastfeeding mothers

- Unable or unwilling to comply with treatment, assessment, or follow-up schedule

- Taking any medications contraindicated with medicines in any trial regimen of the study

- Known allergy to any drug of treatment regimens

- Currently taking part in another trial

- QTc interval > $480 \mathrm{~ms}$

Anticipated sample size: 3900 (1300 in each group)

Interventions

\section{Interventions:}

\section{Levofloxacin + ethambutol 4.5-month combination ATT regimen}

4.5 months of isoniazid, rifampin, pyrazinamide, ethambutol, and levofloxacin 
Dosage: isoniazid $300 \mathrm{mg}$ (given once daily), rifampin $450 \mathrm{mg}$ (less than $50 \mathrm{~kg}$ given once daily) or $600 \mathrm{mg}$ (more than $50 \mathrm{~kg}$ given once daily), pyrazinamide $1500 \mathrm{mg}$ ((less than $50 \mathrm{~kg}$ given once daily) or $30 \mathrm{mg} / \mathrm{kg}$ (more than $50 \mathrm{~kg}$ once daily), ethambutol $750 \mathrm{mg}$ (less than $50 \mathrm{~kg}$ once daily) or $1000 \mathrm{mg}$ (more than $50 \mathrm{~kg}$ once daily), levofloxacin $600 \mathrm{mg}$ (less than $50 \mathrm{~kg}$ given once daily) or 800 $\mathrm{mg}$ (more than $50 \mathrm{~kg}$ once daily)

\section{Ethambutol 4.5-month combination ATT regimen}

4.5 months of isoniazid, rifampin, pyrazinamide, and ethambutol. Dosage of isoniazid, rifampin, pyrazinamide, and ethambutol is same as that of control regimen

\section{Control:}

\section{Standard 6-month ATT regimen}

2 months of isoniazid, rifampin, pyrazinamide, and ethambutol, followed by

4 months of isoniazid and rifampin

Dosage: isoniazid $300 \mathrm{mg}$ (given once daily), rifampin $450 \mathrm{mg}$ (less than $50 \mathrm{~kg}$ given once daily) or $600 \mathrm{mg}$ (more than $50 \mathrm{~kg}$ given once daily), pyrazinamide $1500 \mathrm{mg}$ (less than $50 \mathrm{~kg}$ given once daily) or $30 \mathrm{mg} / \mathrm{kg}$ (more than $50 \mathrm{~kg}$ once daily), ethambutol $750 \mathrm{mg}$ (less than $50 \mathrm{~kg}$ once daily), or 1000 $\mathrm{mg}$ (more than $50 \mathrm{~kg}$, once daily)

\section{Outcomes}

\section{Primary outcome measures:}

- Percentage of participants with TB recurrence/relapse by 24 months after end of treatment

- Percentage of participants with treatment failure at 4.5 months or 6 months after randomization

\section{Secondary outcome measures:}

- Treatment adverse reactions occurring

- Time to sputum smear or culture conversion within intensive phase

- Sputum smear or culture conversion proportion at treatment completion

- Radiological manifestation change in TB lesion or cavity

- Patient adherence rate

\begin{tabular}{ll}
\hline Starting date & August 2016; estimated study completion date: December 2018 \\
\hline Contact information & Shenjie Tang, MD. Beijing Chest Hospital \\
& Email: tangsj1106@sina.com \\
\hline Notes & Study location: China \\
& Registration number: NCT02901288 \\
& Primary sponsors: Beijing Chest Hospital, Hubei Provincial Center for Disease Control and Preven- \\
& tion
\end{tabular}

Abbreviations: AFB: acid-fast bacilli; ALP: alkaline phosphatase; ALT: alanine aminotransferase; ART: antiretroviral therapy; ARV: antiretroviral; AST: aspartate aminotransferase; ATT: anti-tuberculosis treatment; CrCl: creatinine clearance; E: ethambutol; ECG: electrocardiogram; FSH: follicle-stimulating hormone; H: isoniazid; HbAlc: glycosylated haemoglobin; IUCD: intrauterine coil device; LH: luteinizing hormone; M: moxifloxacin; MDR-TB: multi-drug-resistant tuberculosis; MGIT: mycobacterial growth indicator tube; MIC: minimum inhibitory concentration; MTB: Mycobacterium tuberculosis; PK: pharmacokinetics; R: rifampicin; TB: tuberculosis; TEAE: treatment-emergent adverse event; TTP: time to positivity; U-HCG: urine human chorionic gonadotropin; ULN: upper limit of normal; WHO: World Health Organization; XDR: extensively drug-resistant; Z: pyrazinamide. 
DATA AND ANALYSES

Comparison 1. Moxifloxacin-containing 4-month ATT versus standard 6-month ATT regimens

\begin{tabular}{|c|c|c|c|c|}
\hline Outcome or subgroup title & No. of studies & $\begin{array}{l}\text { No. of partici- } \\
\text { pants }\end{array}$ & Statistical method & Effect size \\
\hline 1 Relapse & 3 & 2265 & $\begin{array}{l}\text { Risk Ratio (M-H, Fixed, 95\% } \\
\mathrm{CI})\end{array}$ & $3.56[2.37,5.37]$ \\
\hline 2 Relapse: subgroup analysis & 3 & & $\begin{array}{l}\text { Risk Ratio (M-H, Fixed, 95\% } \\
\mathrm{Cl} \text { ) }\end{array}$ & Subtotals only \\
\hline $\begin{array}{l}2.1 \text { Moxifloxacin replacing ethambu- } \\
\text { tol }\end{array}$ & 2 & 1386 & $\begin{array}{l}\text { Risk Ratio (M-H, Fixed, 95\% } \\
\mathrm{Cl})\end{array}$ & $2.74[1.69,4.43]$ \\
\hline 2.2 Moxifloxacin replacing isoniazid & 2 & 1424 & $\begin{array}{l}\text { Risk Ratio (M-H, Fixed, 95\% } \\
\mathrm{Cl})\end{array}$ & $4.89[3.02,7.92]$ \\
\hline $\begin{array}{l}3 \text { Relapse: sensitivity analysis ac- } \\
\text { counting for missing data }\end{array}$ & 3 & & $\begin{array}{l}\text { Risk Ratio (M-H, Fixed, 95\% } \\
\mathrm{Cl} \text { ) }\end{array}$ & Subtotals only \\
\hline 3.1 Modified-ITT analysis & 3 & 2265 & $\begin{array}{l}\text { Risk Ratio (M-H, Fixed, 95\% } \\
\mathrm{Cl})\end{array}$ & $3.56[2.37,5.37]$ \\
\hline 3.2 Per-protocol analysis & 3 & 2135 & $\begin{array}{l}\text { Risk Ratio (M-H, Fixed, 95\% } \\
\mathrm{CI})\end{array}$ & $3.79[2.48,5.78]$ \\
\hline 3.3 Imputing missing data & 3 & 2524 & $\begin{array}{l}\text { Risk Ratio (M-H, Fixed, 95\% } \\
\mathrm{Cl} \text { ) }\end{array}$ & $3.83[2.58,5.70]$ \\
\hline 4 Death from any cause & 3 & 2760 & $\begin{array}{l}\text { Risk Ratio (M-H, Fixed, 95\% } \\
\mathrm{Cl} \text { ) }\end{array}$ & $1.06[0.65,1.75]$ \\
\hline 5 Treatment discontinuation & 3 & 2335 & $\begin{array}{l}\text { Risk Ratio (M-H, Fixed, 95\% } \\
\mathrm{Cl} \text { ) }\end{array}$ & $1.12[0.78,1.61]$ \\
\hline $\begin{array}{l}6 \text { Positive sputum culture/smear at } \\
8 \text { weeks }\end{array}$ & 3 & 2828 & $\begin{array}{l}\text { Risk Ratio (M-H, Random, } \\
95 \% \mathrm{Cl})\end{array}$ & $0.49[0.22,1.13]$ \\
\hline $\begin{array}{l}\text { 6.1 Moxifloxacin replacing isoniazid } \\
\text { or ethambutol in 4-month ATT regi- } \\
\text { men }\end{array}$ & 2 & 2087 & $\begin{array}{l}\text { Risk Ratio (M-H, Random, } \\
95 \% \mathrm{Cl})\end{array}$ & $0.74[0.45,1.20]$ \\
\hline $\begin{array}{l}\text { 6.2 Moxifloxacin augmenting stan- } \\
\text { dard 6-month ATT regimen }\end{array}$ & 1 & 741 & $\begin{array}{l}\text { Risk Ratio (M-H, Random, } \\
95 \% \mathrm{Cl})\end{array}$ & $0.24[0.15,0.39]$ \\
\hline 7 Treatment failure & 3 & 2282 & $\begin{array}{l}\text { Risk Ratio (M-H, Fixed, 95\% } \\
\mathrm{Cl})\end{array}$ & $0.71[0.33,1.52]$ \\
\hline 8 Acquired drug resistance & 3 & 2282 & $\begin{array}{l}\text { Risk Ratio (M-H, Fixed, 95\% } \\
\mathrm{Cl} \text { ) }\end{array}$ & $0.33[0.08,1.31]$ \\
\hline 9 Serious adverse events & 4 & 3548 & $\begin{array}{l}\text { Risk Ratio (M-H, Fixed, 95\% } \\
\text { Cl) }\end{array}$ & $0.97[0.74,1.27]$ \\
\hline $\begin{array}{l}\text { 9.1 Moxifloxacin replacing standard } \\
\text { drugs in 4-month ATT regimens }\end{array}$ & 3 & 2760 & $\begin{array}{l}\text { Risk Ratio (M-H, Fixed, 95\% } \\
\mathrm{Cl})\end{array}$ & $0.96[0.72,1.26]$ \\
\hline
\end{tabular}




\begin{tabular}{lllll}
\hline Outcome or subgroup title & No. of studies & $\begin{array}{l}\text { No. of partici- } \\
\text { pants }\end{array}$ & Statistical method & Effect size \\
\hline $\begin{array}{l}\text { 9.2 Moxifloxacin augmenting stan- } \\
\text { dard 6-month ATT regimens }\end{array}$ & 1 & 788 & $\begin{array}{l}\text { Risk Ratio (M-H, Fixed, 95\% } \\
\text { Cl) }\end{array}$ & 1.17 [0.45, 3.06] \\
\hline
\end{tabular}

Analysis 1.1. Comparison 1 Moxifloxacin-containing 4-month ATT versus standard 6-month ATT regimens, Outcome 1 Relapse.

\begin{tabular}{|c|c|c|c|c|c|}
\hline Study or subgroup & $\begin{array}{l}\text { Four-month } \\
\text { regimen } \\
n / N\end{array}$ & $\begin{array}{l}\text { Six-month } \\
\text { regimen } \\
n / N\end{array}$ & $\begin{array}{c}\text { Risk Ratio } \\
\text { M-H, Fixed, 95\% Cl }\end{array}$ & Weight & $\begin{array}{c}\text { Risk Ratio } \\
\text { M-H, Fixed, } 95 \% \mathrm{Cl}\end{array}$ \\
\hline Gillespie 2014 & $110 / 1119$ & $13 / 555$ & $\because$ & $54.95 \%$ & $4.2[2.38,7.39]$ \\
\hline Jawahar 2013 & $11 / 108$ & $10 / 155$ & $\longrightarrow$ & $25.97 \%$ & $1.58[0.7,3.59]$ \\
\hline Jindani 2014 & $27 / 165$ & $6 / 163$ & $\longrightarrow$ & $19.09 \%$ & $4.45[1.89,10.48]$ \\
\hline \multicolumn{6}{|c|}{ Total events: 148 (Four-month regimen), 29 (Six-month regimen) } \\
\hline \multicolumn{6}{|c|}{ Heterogeneity: $\mathrm{Tau}^{2}=0 ; \mathrm{Chi}^{2}=4.36, \mathrm{df}=2(\mathrm{P}=0.11) ; \mathrm{I}^{2}=54.13 \%$} \\
\hline \multicolumn{6}{|c|}{ Test for overall effect: $Z=6.09(P<0.0001)$} \\
\hline
\end{tabular}

Analysis 1.2. Comparison 1 Moxifloxacin-containing 4-month ATT versus standard 6-month ATT regimens, Outcome 2 Relapse: subgroup analysis.

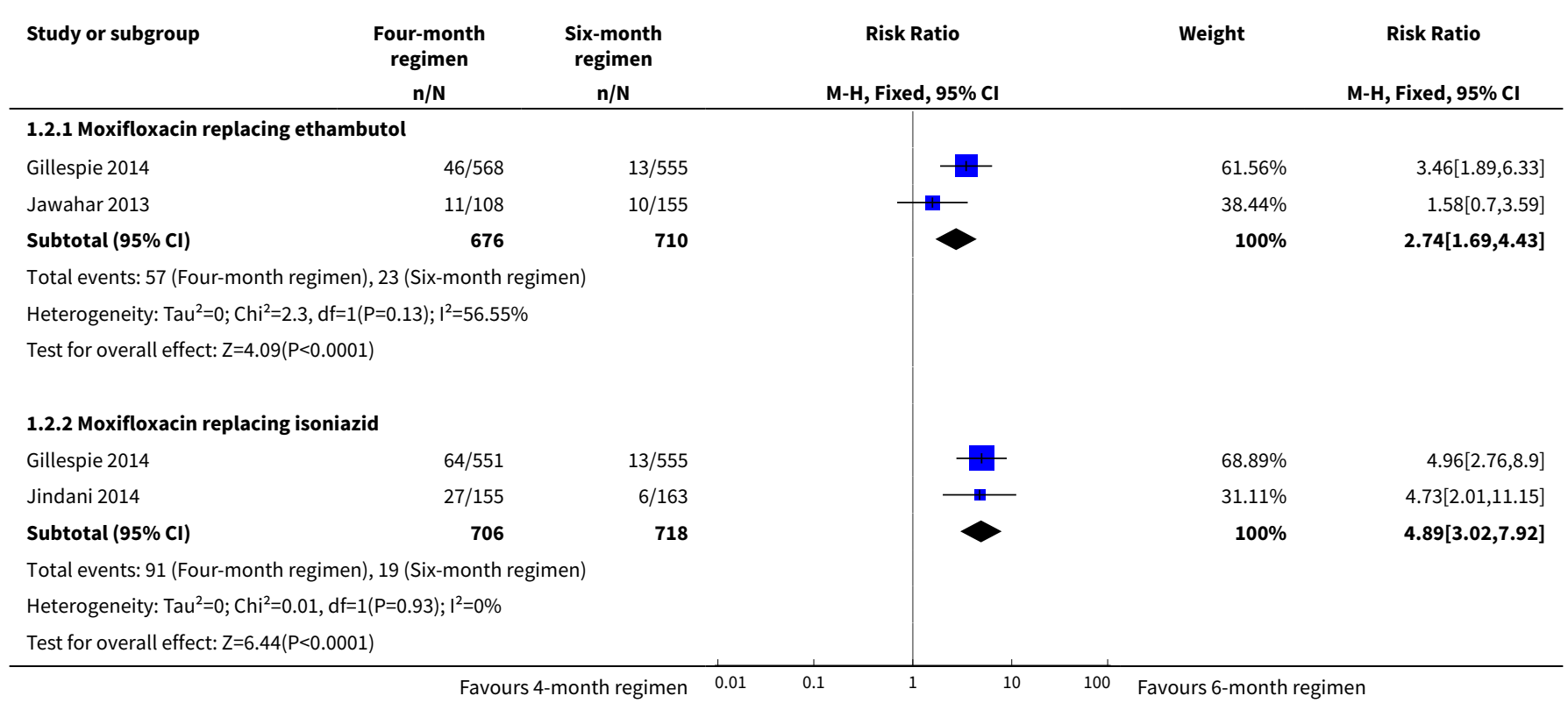


Analysis 1.3. Comparison 1 Moxifloxacin-containing 4-month ATT versus standard 6month ATT regimens, Outcome 3 Relapse: sensitivity analysis accounting for missing data.

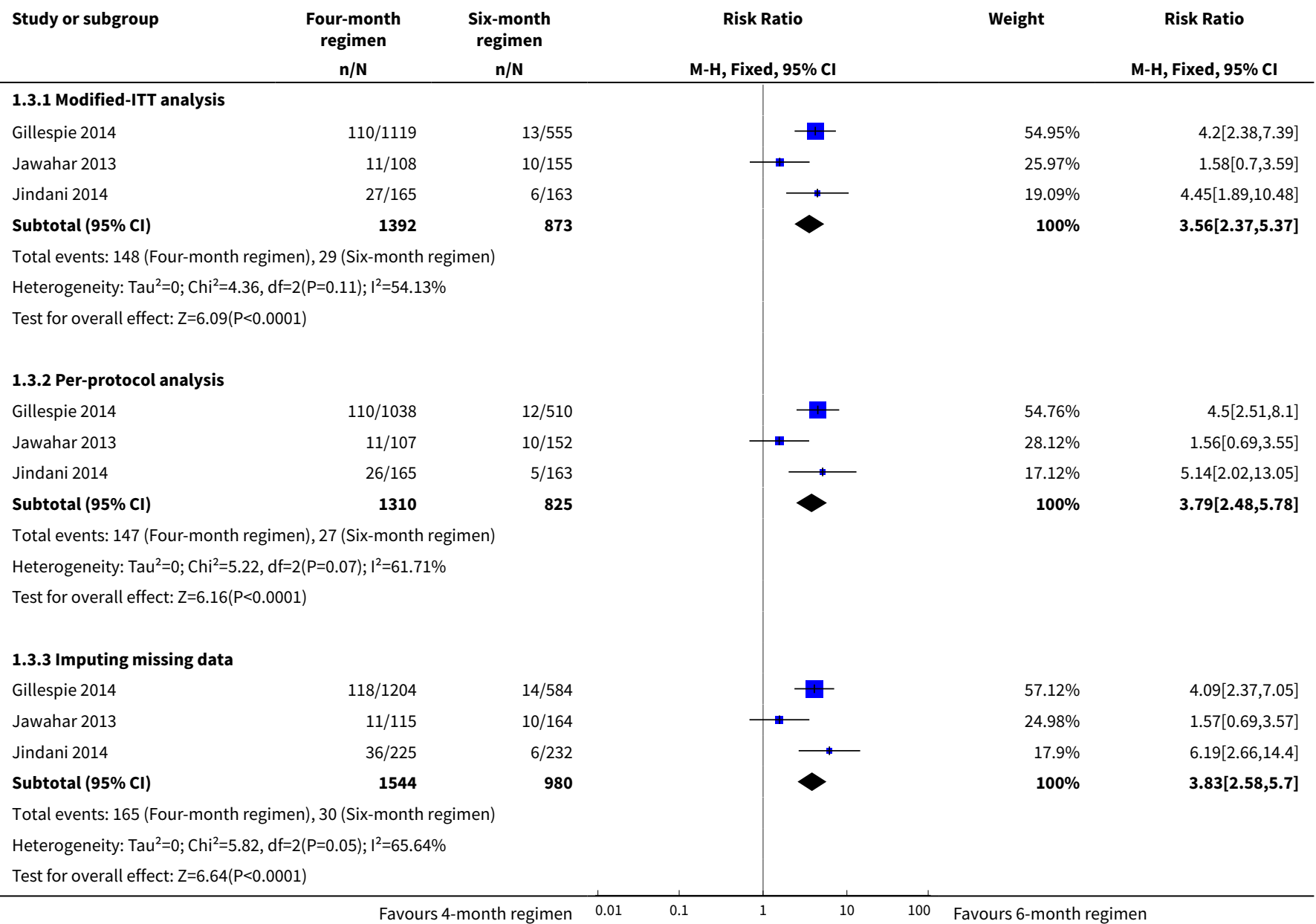

\section{Analysis 1.4. Comparison 1 Moxifloxacin-containing 4-month ATT versus} standard 6-month ATT regimens, Outcome 4 Death from any cause.

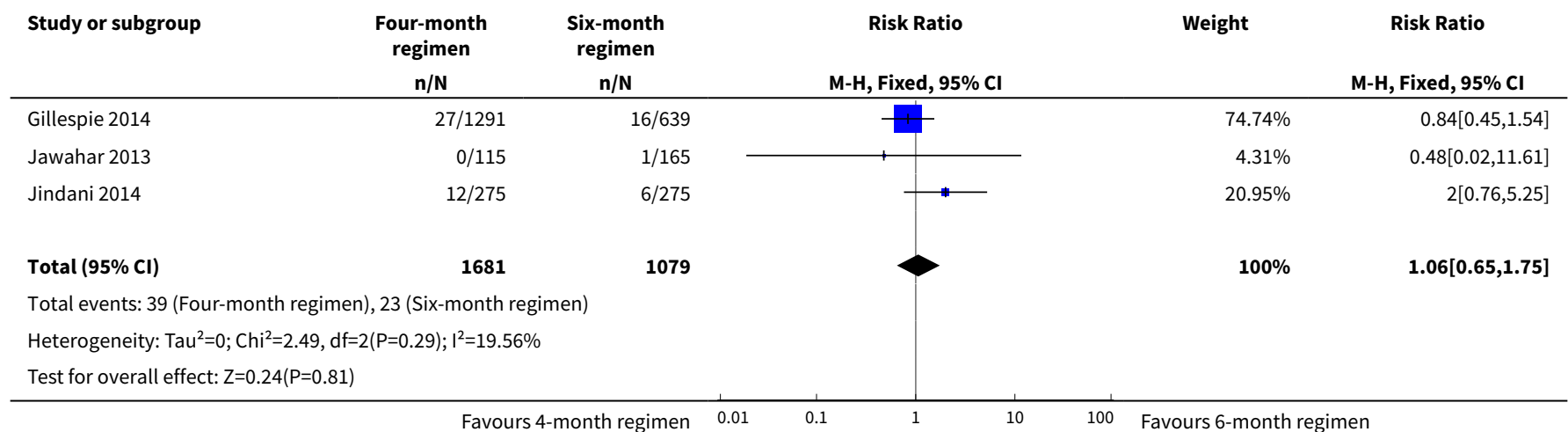

Shortened treatment regimens versus the standard regimen for drug-sensitive pulmonary tuberculosis (Review) 
Analysis 1.5. Comparison 1 Moxifloxacin-containing 4-month ATT versus standard 6-month ATT regimens, Outcome 5 Treatment discontinuation.

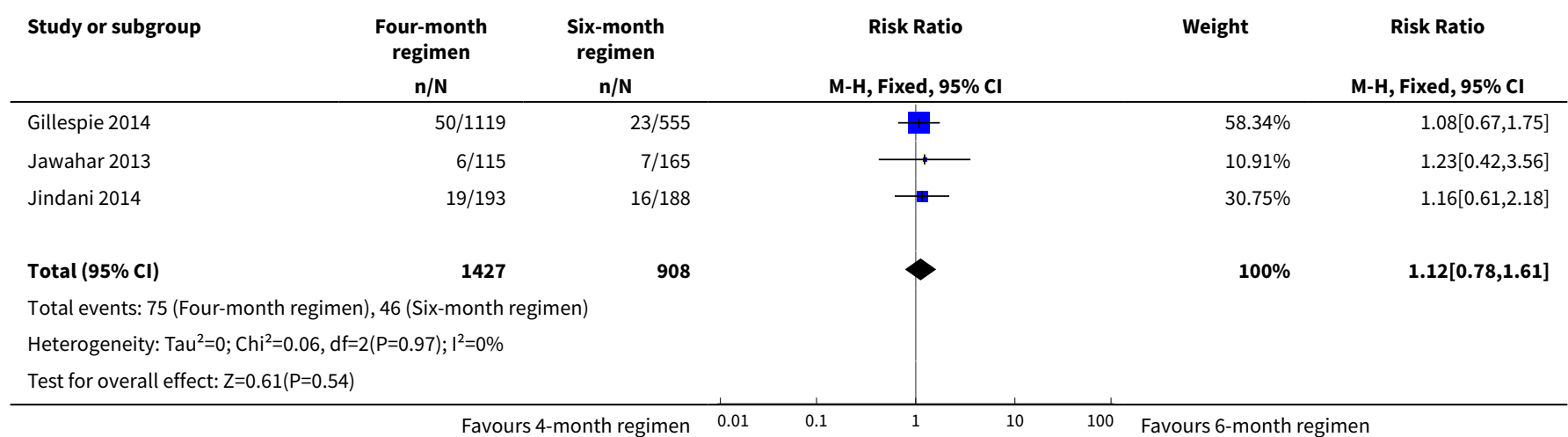

\section{Analysis 1.6. Comparison 1 Moxifloxacin-containing 4-month ATT versus standard 6-month ATT regimens, Outcome 6 Positive sputum culture/smear at 8 weeks.}

\begin{tabular}{|c|c|c|c|c|c|}
\hline Study or subgroup & $\begin{array}{c}\text { Four-month } \\
\text { regimen } \\
n / N \\
\end{array}$ & $\begin{array}{l}\text { Six-month } \\
\text { regimen } \\
n / N \\
\end{array}$ & M-H, Random, 95\% Cl & Weight & $\begin{array}{c}\text { Risk Ratio } \\
\text { M-H, Random, } 95 \% \mathrm{CI}\end{array}$ \\
\hline \multicolumn{6}{|c|}{$\begin{array}{l}\text { 1.6.1 Moxifloxacin replacing isoniazid or ethambutol in 4-month ATT } \\
\text { regimen }\end{array}$} \\
\hline Gillespie 2014 & $140 / 1221$ & $77 / 600$ & 典 & $35.71 \%$ & $0.89[0.69,1.16]$ \\
\hline Jawahar 2013 & $14 / 112$ & $36 / 154$ & & $31.55 \%$ & $0.53[0.3,0.94]$ \\
\hline Subtotal $(95 \% \mathrm{Cl})$ & 1333 & 754 & & $67.26 \%$ & $0.74[0.45,1.2]$ \\
\hline \multicolumn{6}{|c|}{ Total events: 154 (Four-month regimen), 113 (Six-month regimen) } \\
\hline \multicolumn{6}{|c|}{ Heterogeneity: $\mathrm{Tau}^{2}=0.08 ; \mathrm{Chi}^{2}=2.6, \mathrm{df}=1(\mathrm{P}=0.11) ; \mathrm{I}^{2}=61.55 \%$} \\
\hline \multicolumn{6}{|c|}{ Test for overall effect: $Z=1.23(P=0.22)$} \\
\hline \multicolumn{6}{|c|}{ 1.6.2 Moxifloxacin augmenting standard 6-month ATT regimen } \\
\hline Velayutham 2014 & $27 / 590$ & $29 / 151$ & $\rightarrow-$ & $32.74 \%$ & $0.24[0.15,0.39]$ \\
\hline Subtotal $(95 \% \mathrm{Cl})$ & 590 & 151 & & $32.74 \%$ & $0.24[0.15,0.39]$ \\
\hline \multicolumn{6}{|c|}{ Heterogeneity: Not applicable } \\
\hline \multicolumn{6}{|c|}{ Test for overall effect: $Z=5.71(P<0.0001)$} \\
\hline Total $(95 \% \mathrm{Cl})$ & 1923 & 905 & & $100 \%$ & $0.49[0.22,1.13]$ \\
\hline \multicolumn{6}{|c|}{ Total events: 181 (Four-month regimen), 142 (Six-month regimen) } \\
\hline \multicolumn{6}{|c|}{ Heterogeneity: $\mathrm{Tau}^{2}=0.48 ; \mathrm{Chi}^{2}=22.17, \mathrm{df}=2(\mathrm{P}<0.0001) ; \mathrm{I}^{2}=90.98 \%$} \\
\hline \multicolumn{6}{|c|}{ Test for overall effect: $Z=1.67(P=0.09)$} \\
\hline Test for subgroup dif & $2, \mathrm{df}=1(\mathrm{P}=0), \mathrm{I}^{2}=$ & & & & \\
\hline
\end{tabular}


Analysis 1.7. Comparison 1 Moxifloxacin-containing 4-month ATT versus standard 6-month ATT regimens, Outcome 7 Treatment failure.

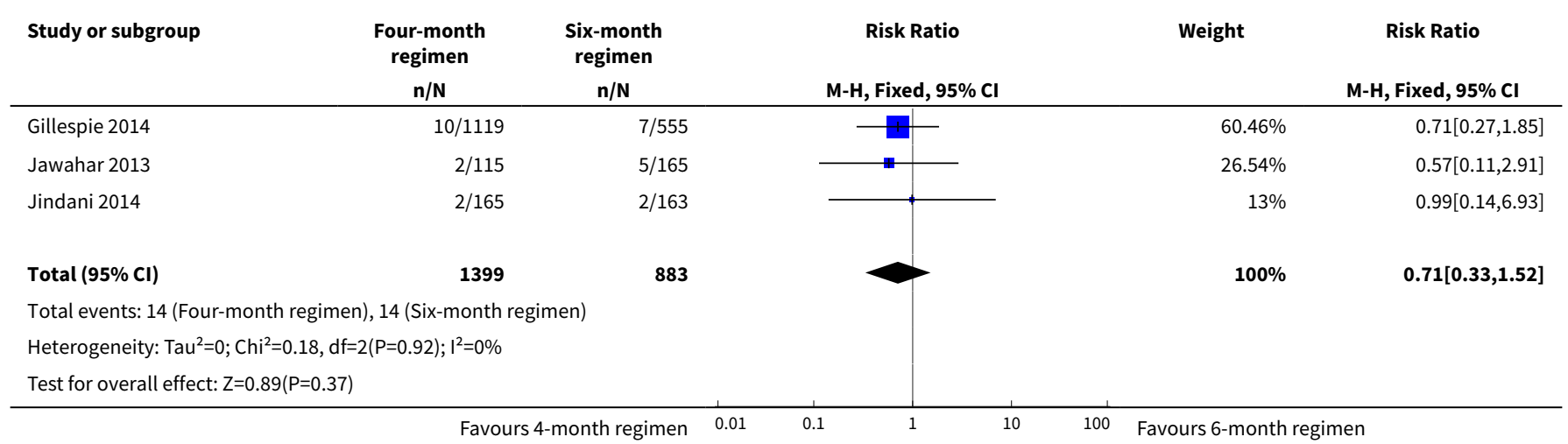

Analysis 1.8. Comparison 1 Moxifloxacin-containing 4-month ATT versus standard 6-month ATT regimens, Outcome 8 Acquired drug resistance.

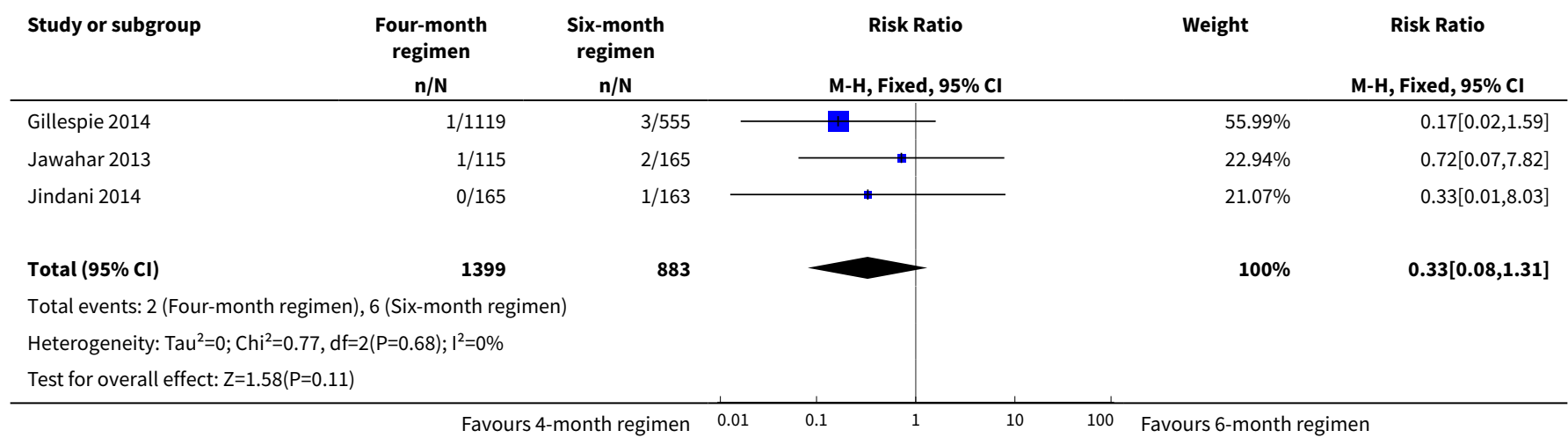

\section{Analysis 1.9. Comparison 1 Moxifloxacin-containing 4-month ATT versus standard 6-month ATT regimens, Outcome 9 Serious adverse events.}

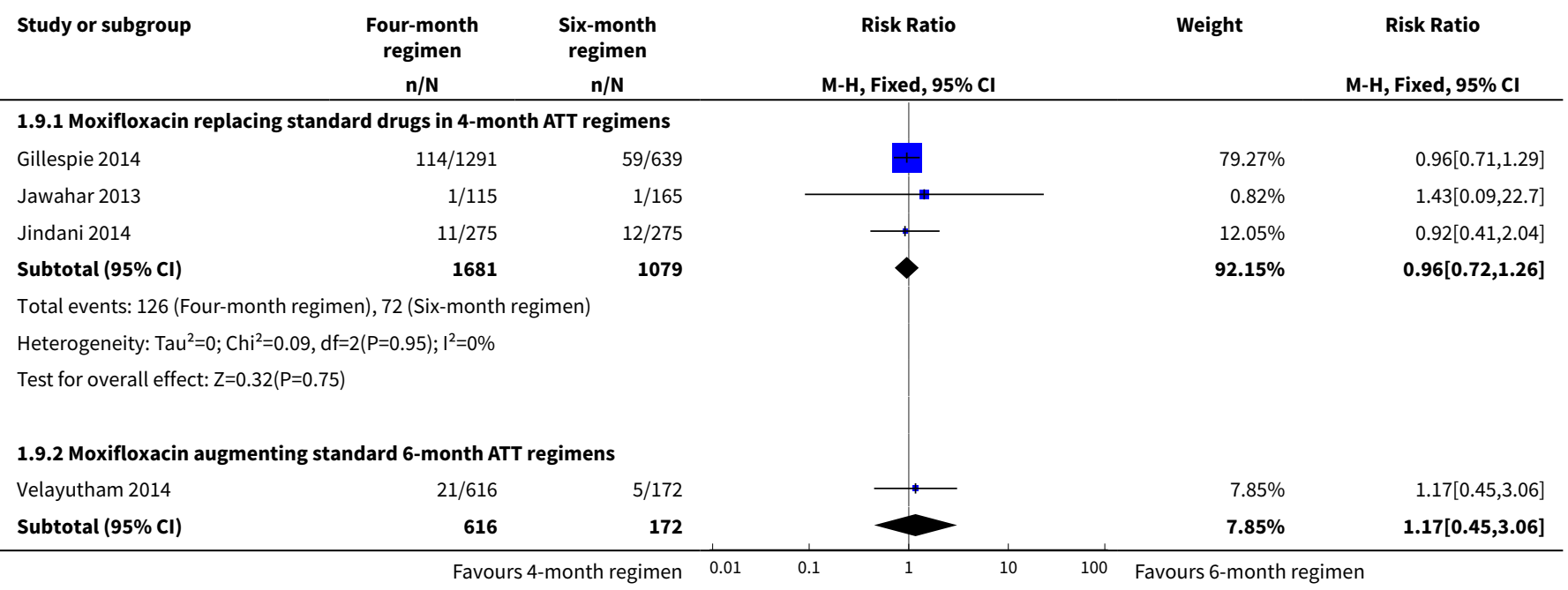




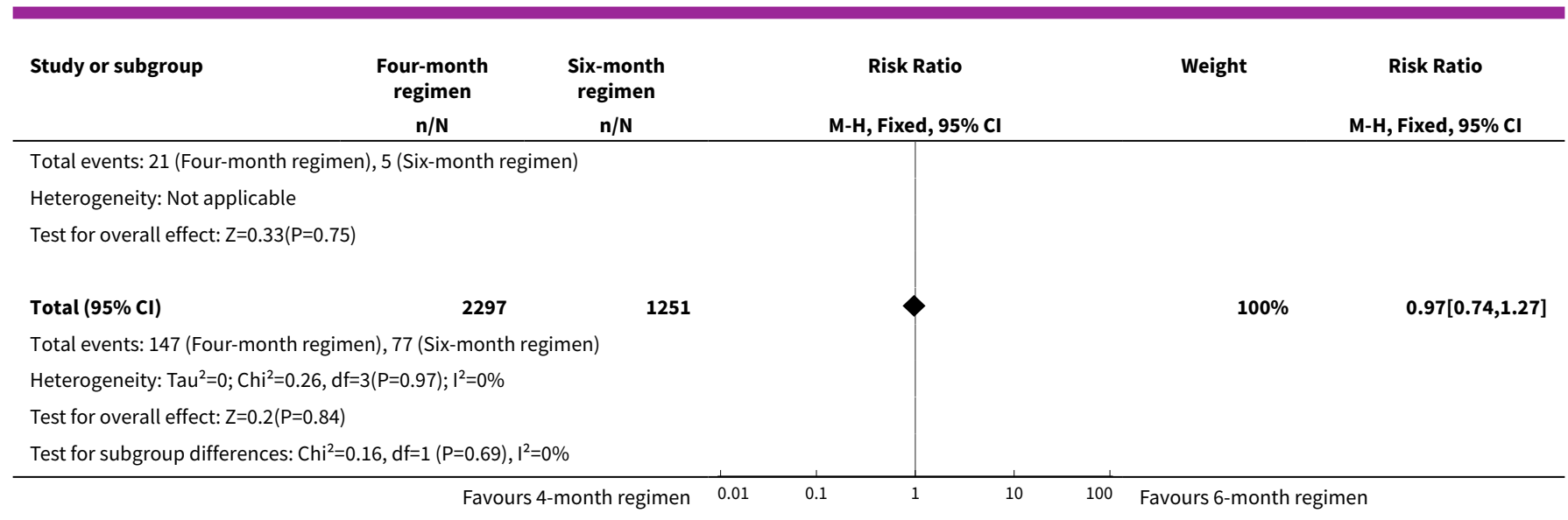

\section{Comparison 2. Gatifloxacin-containing 4-month ATT versus standard 6-month ATT regimens}

\begin{tabular}{|c|c|c|c|c|}
\hline Outcome or subgroup title & No. of studies & $\begin{array}{l}\text { No. of partici- } \\
\text { pants }\end{array}$ & Statistical method & Effect size \\
\hline 1 Relapse & 2 & 1633 & Risk Ratio (M-H, Fixed, 95\% Cl) & $2.11[1.56,2.84]$ \\
\hline $\begin{array}{l}2 \text { Relapse: sensitivity analysis ac- } \\
\text { counting for missing data }\end{array}$ & 2 & & Risk Ratio (M-H, Fixed, 95\% Cl) & Subtotals only \\
\hline 2.1 Modified-ITT analysis & 2 & 1633 & Risk Ratio (M-H, Fixed, 95\% Cl) & $2.11[1.56,2.84]$ \\
\hline 2.2 Per-protocol analysis & 2 & 1525 & Risk Ratio (M-H, Fixed, 95\% Cl) & $2.11[1.55,2.87]$ \\
\hline $\begin{array}{l}\text { 2.3 Modified-ITT analysis (all el- } \\
\text { igible participants - imputing } \\
\text { missing data) }\end{array}$ & 2 & 1851 & Risk Ratio (M-H, Fixed, 95\% Cl) & $2.01[1.53,2.63]$ \\
\hline 3 Death from any cause & 2 & 1886 & Risk Ratio (M-H, Fixed, 95\% Cl) & $0.90[0.53,1.53]$ \\
\hline 4 Treatment discontinuation & 2 & 1657 & Risk Ratio (M-H, Fixed, 95\% Cl) & $0.70[0.46,1.08]$ \\
\hline $\begin{array}{l}5 \text { Positive sputum culture at } 8 \\
\text { weeks }\end{array}$ & 2 & 1818 & Risk Ratio (M-H, Fixed, 95\% Cl) & $0.99[0.80,1.23]$ \\
\hline 6 Treatment failure & 2 & 1657 & Risk Ratio (M-H, Fixed, 95\% Cl) & $0.93[0.51,1.70]$ \\
\hline 7 Acquired drug resistance & 1 & 301 & Risk Ratio (M-H, Fixed, 95\% Cl) & $0.24[0.01,5.01]$ \\
\hline 8 Serious adverse events & 2 & 1993 & Risk Ratio (M-H, Fixed, 95\% Cl) & $1.02[0.58,1.77]$ \\
\hline
\end{tabular}


Analysis 2.1. Comparison 2 Gatifloxacin-containing 4-month ATT versus standard 6-month ATT regimens, Outcome 1 Relapse.

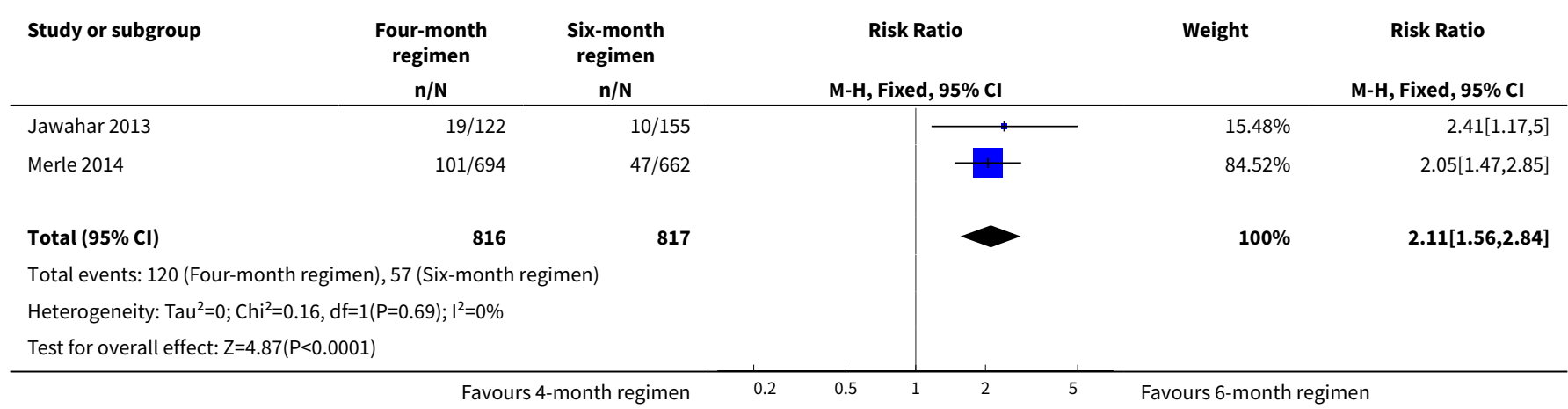

Analysis 2.2. Comparison 2 Gatifloxacin-containing 4-month ATT versus standard 6month ATT regimens, Outcome 2 Relapse: sensitivity analysis accounting for missing data.

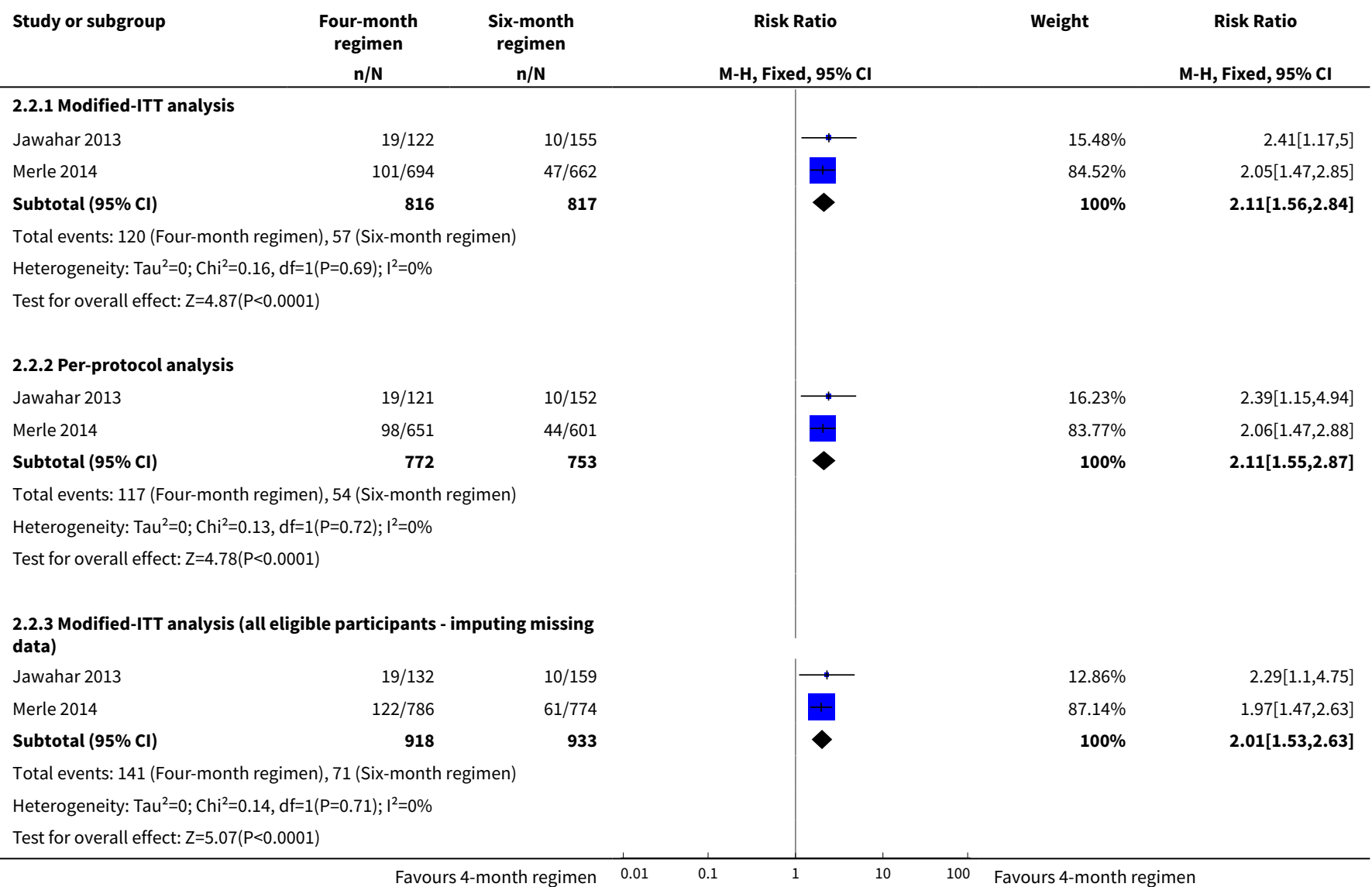


Analysis 2.3. Comparison 2 Gatifloxacin-containing 4-month ATT versus standard 6-month ATT regimens, Outcome 3 Death from any cause.

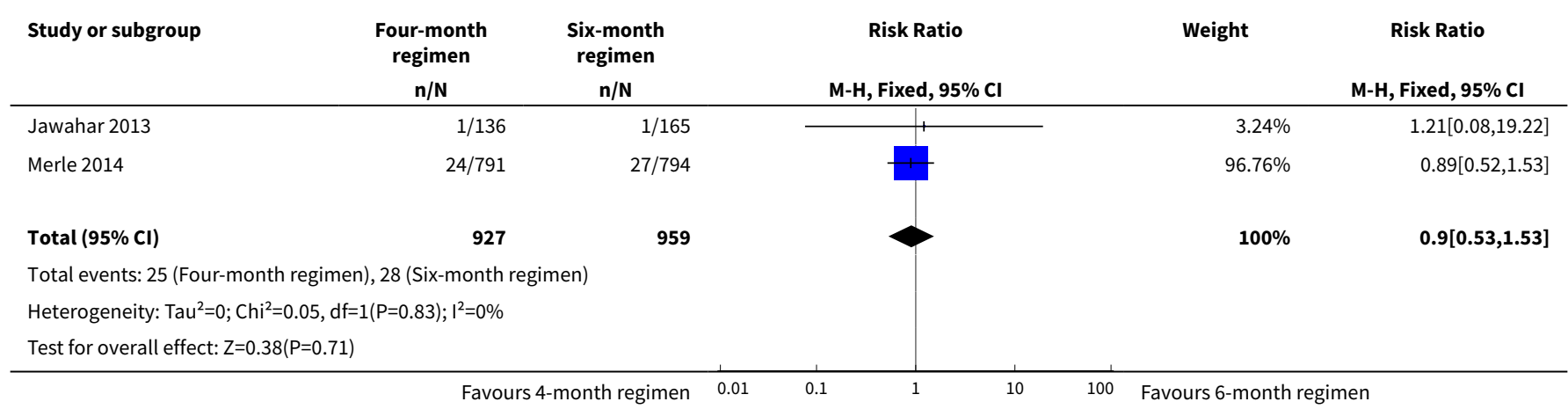

Analysis 2.4. Comparison 2 Gatifloxacin-containing 4-month ATT versus standard 6-month ATT regimens, Outcome 4 Treatment discontinuation.

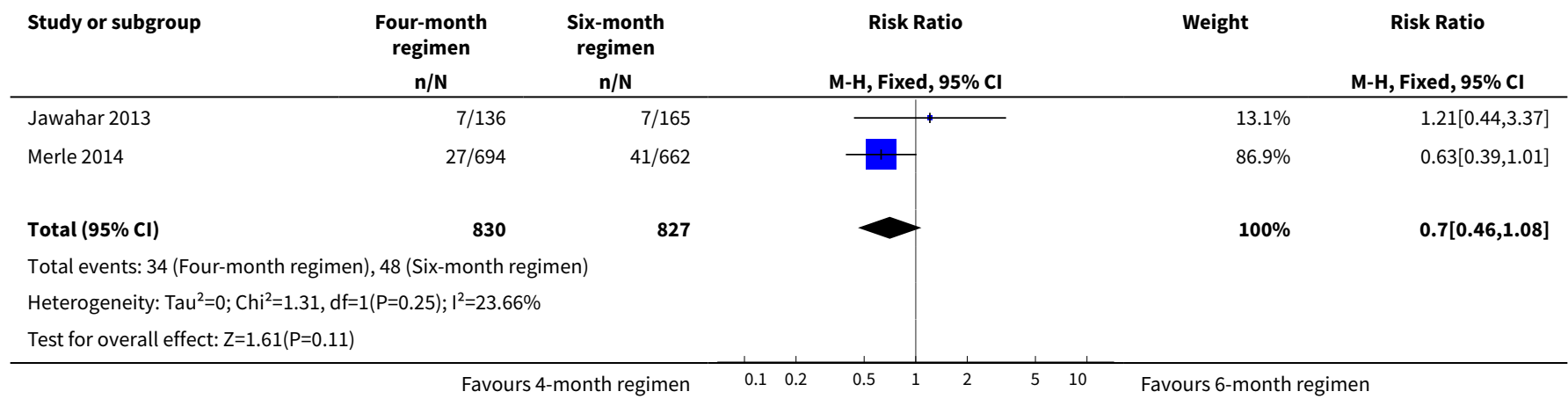

Analysis 2.5. Comparison 2 Gatifloxacin-containing 4-month ATT versus standard 6-month ATT regimens, Outcome 5 Positive sputum culture at 8 weeks.

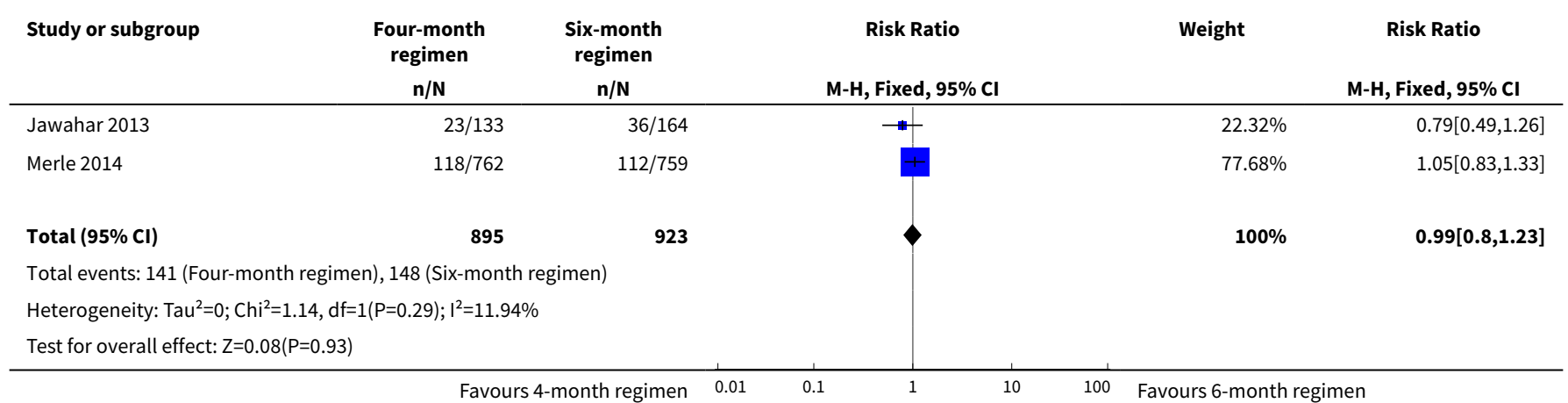


Analysis 2.6. Comparison 2 Gatifloxacin-containing 4-month ATT versus standard 6-month ATT regimens, Outcome 6 Treatment failure.

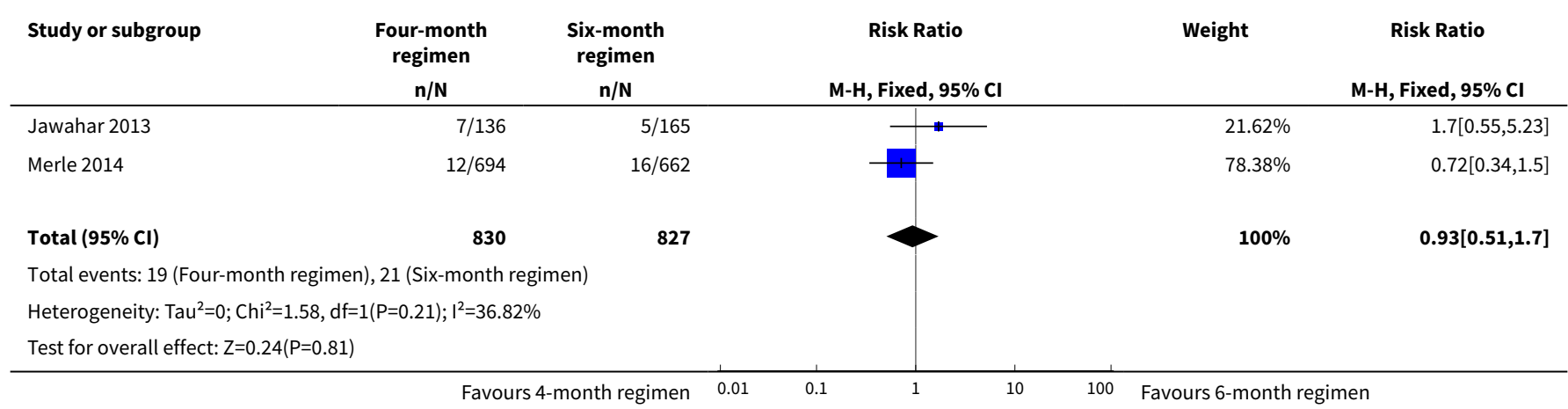

Analysis 2.7. Comparison 2 Gatifloxacin-containing 4-month ATT versus standard 6-month ATT regimens, Outcome 7 Acquired drug resistance.

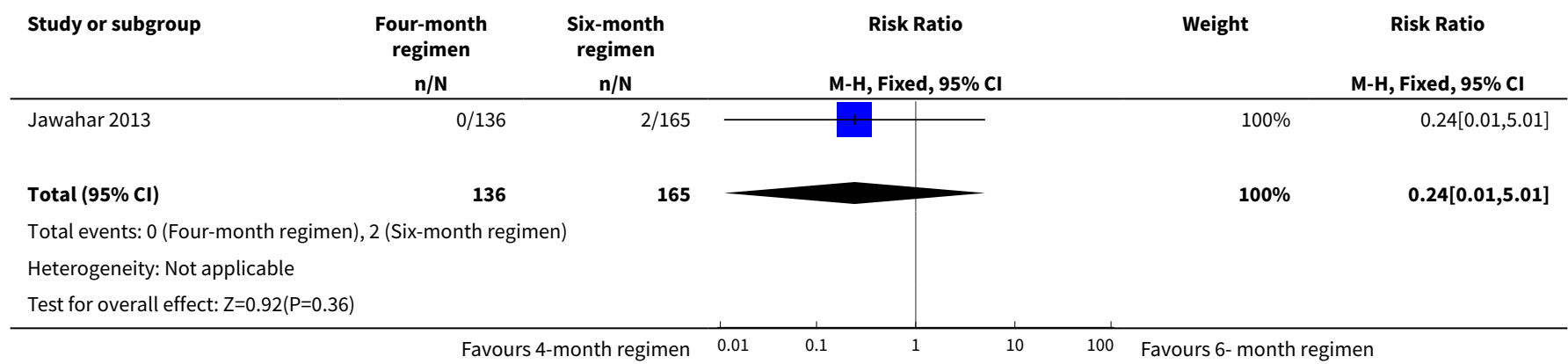

Analysis 2.8. Comparison 2 Gatifloxacin-containing 4-month ATT versus standard 6-month ATT regimens, Outcome 8 Serious adverse events.

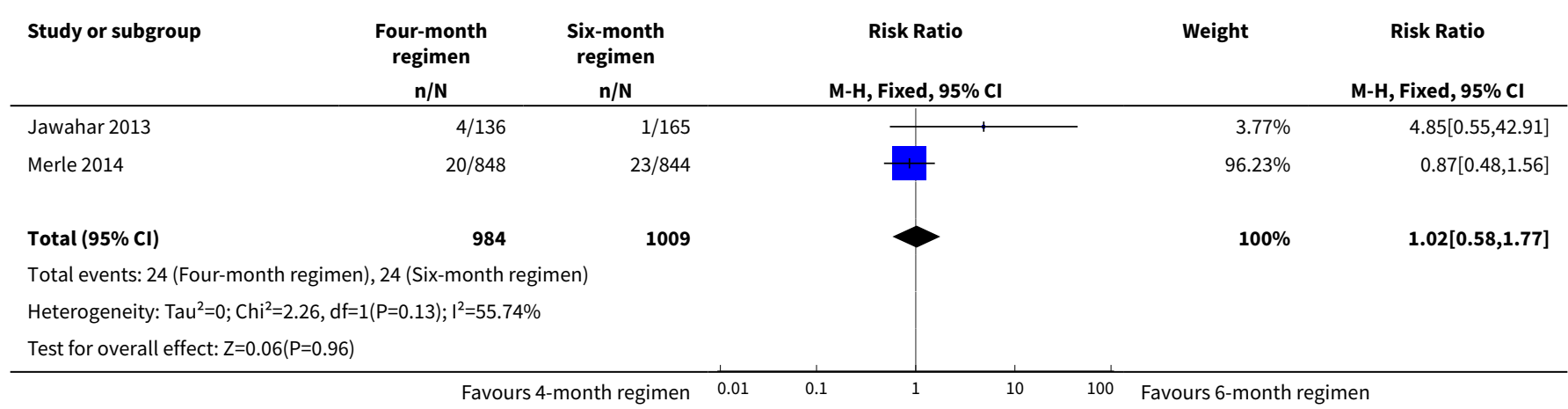


ADDITIONAL TABLES

Table 1. Summary of outcomes in included studies

\begin{tabular}{|c|c|c|c|c|c|}
\hline $\begin{array}{l}\text { Study ID } \\
\text { (Acronym) }\end{array}$ & $\begin{array}{l}\text { Gillespie } 2014 \\
\text { (REMoxTB) }\end{array}$ & Jawahar 2013 & $\begin{array}{l}\text { Jindani } 2014 \\
\text { (RIFAQUIN) }\end{array}$ & $\begin{array}{l}\text { Merle } 2014 \\
\text { (OFLOTUB) }\end{array}$ & Velayutham 2014 \\
\hline Setting & $\begin{array}{l}\text { Multiple sites in Africa (Kenya, } \\
\text { South Africa, Tanzania, } \\
\text { Zambia), Asia (China, India, } \\
\text { Malaysia Thailand), Latin } \\
\text { America (Mexico) }\end{array}$ & 6 sites in 2 cities in India & $\begin{array}{l}6 \text { cities in } 4 \text { countries in Africa } \\
\text { (Botswana, South Africa, Zambia, } \\
\text { Zimbabwe) }\end{array}$ & $\begin{array}{l}5 \text { countries in Africa (Benin, } \\
\text { Guinea, Kenya, Senegal, } \\
\text { South Africa) }\end{array}$ & 2 cities in India \\
\hline \multicolumn{6}{|c|}{ Participants } \\
\hline $\begin{array}{l}\text { Number } \\
\text { random- } \\
\text { ized }\end{array}$ & 1931 & 429 & 827 & 1836 & 801 \\
\hline Age & Adults (> 18 years) & Adults (> 18 years) & Adults (> 18 years) & Adults (18 to 65 years) & Adults (> 18 years) \\
\hline $\begin{array}{l}\text { HIV infec- } \\
\text { tion }\end{array}$ & $\begin{array}{l}\text { Included (if CD4 count }>250 \\
\text { cells/ } \mu \mathrm{L} \text { and not on ART); } 110 \\
(7 \%)\end{array}$ & Excluded & $\begin{array}{l}\text { Included (if CD4 count }>150 / \mathrm{mm}^{3} \\
\text { and not on ART; } 158(27 \%)\end{array}$ & $\begin{array}{l}\text { Included if not stage } 3 \text { or } 4 \\
\text { disease and not on ART; } 304 \\
(17 \%)\end{array}$ & Excluded \\
\hline $\begin{array}{l}\text { Diagnosis } \\
\text { of TB }\end{array}$ & $\begin{array}{l}\text { Positive sputum smears on } 2 \\
\text { occasions } \\
\text { Culture-confirmed suscep- } \\
\text { tibility to rifampicin, isoni- } \\
\text { azid, pyrazinamide, and moxi- } \\
\text { floxacin }\end{array}$ & $\begin{array}{l}\text { Newly diagnosed pul- } \\
\text { monary TB with at least } 2 \\
\text { positive sputum cultures. } \\
\text { Confirmed by culture and } \\
\text { MDR-TB excluded, sus- } \\
\text { ceptibility to ofloxacin (as } \\
\text { proxy for moxifloxacin) }\end{array}$ & $\begin{array}{l}2 \text { sputum samples that were pos- } \\
\text { itive for tubercle bacilli on direct } \\
\text { smear microscopy } \\
\text { No resistance to isoniazid, ri- } \\
\text { fampicin, or moxifloxacin }\end{array}$ & $\begin{array}{l}\text { Acid-fast bacilli in } 2 \text { con- } \\
\text { secutive sputum smears; } \\
\text { confirmed by culture (solid } \\
\text { medium) and drug sensitiv- } \\
\text { ity tests to rifampicin, isoni- } \\
\text { azid, ethambutol, strepto- } \\
\text { mycin, and gatifloxacin }\end{array}$ & $\begin{array}{l}2 \text { positive sputum smear } \\
\text { smears for tuberculosis. } \\
\text { Culture-confirmed and } \\
\text { MDR-TB ruled out; sus- } \\
\text { ceptible to ofloxacin (as } \\
\text { proxy for moxifloxacin) }\end{array}$ \\
\hline
\end{tabular}

Intervention(s) and comparator

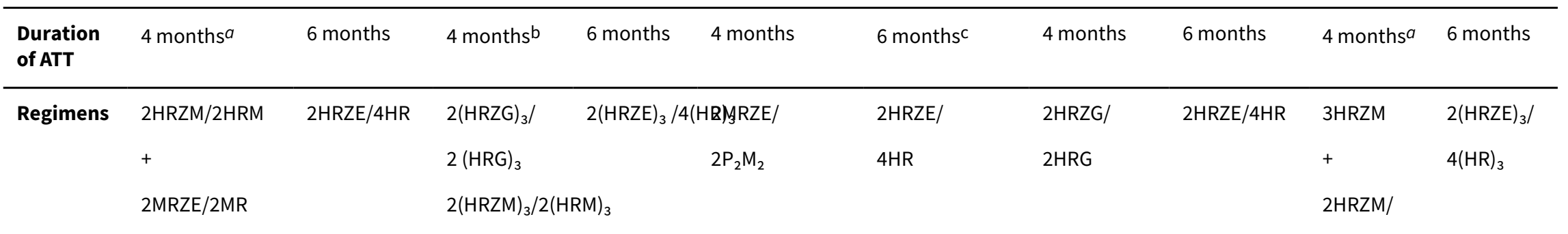




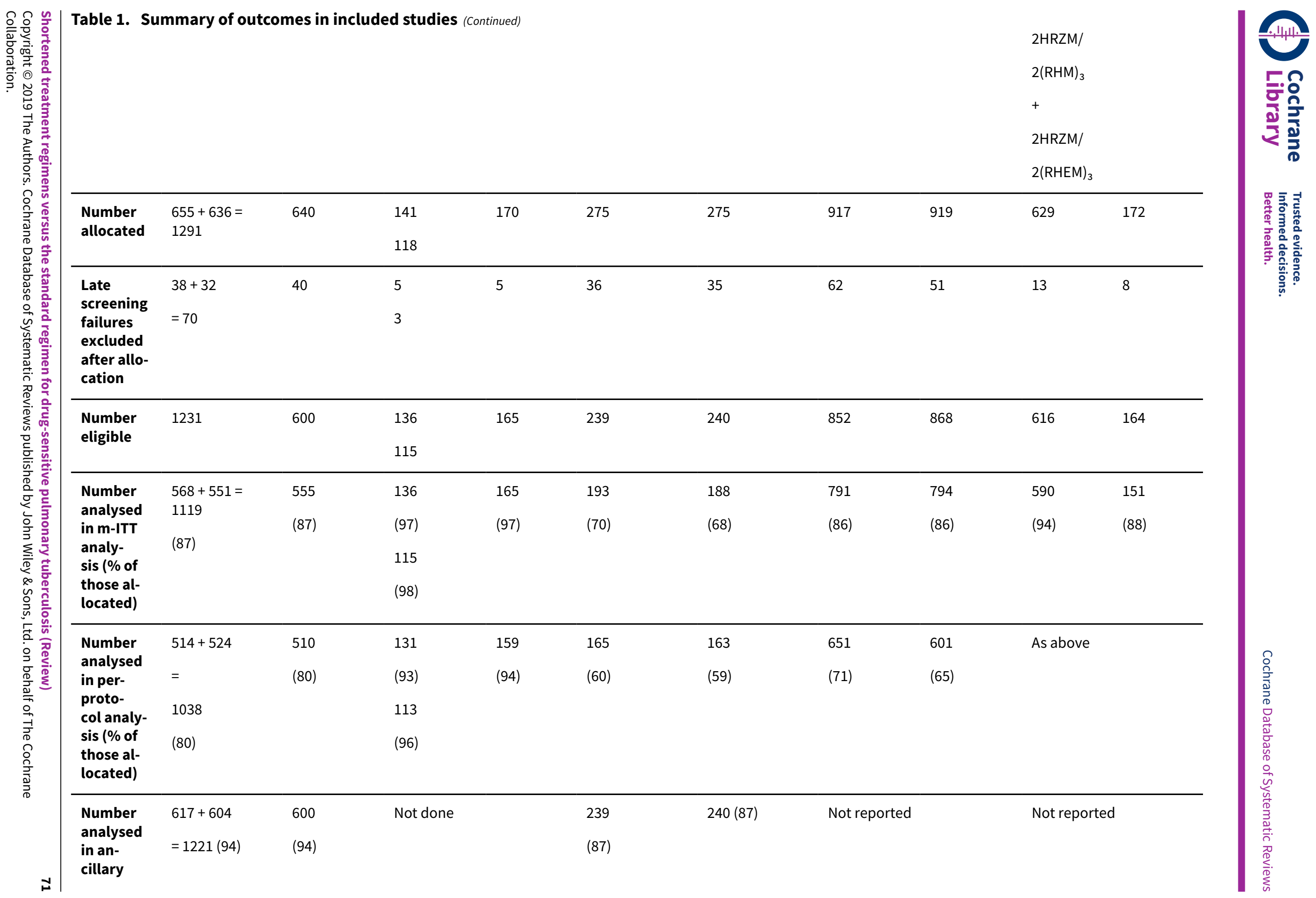




\begin{tabular}{|c|c|c|c|c|c|}
\hline $\begin{array}{l}\text { analysis } \\
\text { (ITT) }(\% \\
\text { of those } \\
\text { allocated) }\end{array}$ & & & & & \\
\hline \multicolumn{6}{|c|}{ Outcomes reported } \\
\hline Relapse & $\begin{array}{l}\text { Relapse within } 18 \text { months after } \\
\text { randomization in those with } \\
\text { negative culture with treat- } \\
\text { ment. Relapse strains were } \\
\text { those shown to be identical on } \\
\text { 24-locus MIRU analysis } \\
\text { LJ solid media and MGIT liquid } \\
\text { media used for culture }\end{array}$ & $\begin{array}{l}\text { Recurrence of TB over } 24 \\
\text { months after treatment in } \\
\text { those with a favourable re- } \\
\text { sponse with treatment: ei- } \\
\text { ther bacteriologic recur- } \\
\text { rence (LJ solid media) or } \\
\text { clinical/radiologic recur- } \\
\text { rence } \\
\text { Relapse not differentiat- } \\
\text { ed from re-infection but } \\
\text { majority occurred within } 6 \\
\text { months after treatment }\end{array}$ & $\begin{array}{l}\text { Relapse within } 12 \text { to } 18 \text { months } \\
\text { after treatment. Two positive cul- } \\
\text { tures within a period of } 4 \text { months } \\
\text { without an intervening negative } \\
\text { culture). Re-infections differenti- } \\
\text { ated from relapse through geno- } \\
\text { typing (MIRU-VNTRs) } \\
\text { LJ solid media used for culture in } \\
\text { some centres, MGIT liquid media } \\
\text { in others, and both in some cen- } \\
\text { tres }\end{array}$ & $\begin{array}{l}\text { Recurrence of TB over } 24 \\
\text { months after treatment } \\
\text { proven bacteriologically ( } 2 \\
\text { consecutive positive spu- } \\
\text { tum samples a day apart) or } \\
\text { clinically } \\
\text { Genotyping (MIRU-VNTRs) } \\
\text { results available for only } \\
70 / 140 \text { (55\%) of those with } \\
\text { culture confirmed recur- } \\
\text { rence. Most were relapses }\end{array}$ & Not reported \\
\hline Deaths & $\begin{array}{l}\text { All deaths } \\
\text { TB deaths }\end{array}$ & $\begin{array}{l}\text { Reported (only non-TB } \\
\text { deaths occurred) }\end{array}$ & $\begin{array}{l}\text { All deaths } \\
\text { TB deaths }\end{array}$ & $\begin{array}{l}\text { Death during treatment } \\
\text { Death after treatment }\end{array}$ & Not reported \\
\hline $\begin{array}{l}\text { Treat- } \\
\text { ment dis- } \\
\text { continua- } \\
\text { tion }\end{array}$ & $\begin{array}{l}\text { Includes those who did not } \\
\text { complete treatment, relocat- } \\
\text { ed, or withdrew consent }\end{array}$ & $\begin{array}{l}\text { Includes those who did } \\
\text { not complete treatment } \\
\text { and those lost to fol- } \\
\text { low-up }\end{array}$ & $\begin{array}{l}\text { Includes change in treatment } \\
\text { due to adverse events, loss to } \\
\text { follow-up, and other treatment } \\
\text { changes }\end{array}$ & $\begin{array}{l}\text { Includes those who with- } \\
\text { drew consent during treat- } \\
\text { ment and dropouts }\end{array}$ & $\begin{array}{l}\text { Reported but disag- } \\
\text { gregated data for each } \\
\text { group not available }\end{array}$ \\
\hline $\begin{array}{l}\text { Positive } \\
\text { smear/ } \\
\text { sputum } \\
\text { culture at } \\
2 \text { months }\end{array}$ & $\begin{array}{l}\text { Reported using LJ solid media } \\
\text { (used in this review) and MGIT } \\
\text { liquid media for all random- } \\
\text { ized participants excluding late } \\
\text { screening failures }\end{array}$ & $\begin{array}{l}\text { Reported using LJ solid } \\
\text { media for all randomized } \\
\text { participants excluding late } \\
\text { screening failures }\end{array}$ & $\begin{array}{l}\text { Reported but disaggregated da- } \\
\text { ta for moxifloxacin 4-month and } \\
6 \text {-month treatment groups not } \\
\text { available } \\
\text { Data also not available for all par- } \\
\text { ticipants from LJ media }\end{array}$ & $\begin{array}{l}\text { Reported for } 752 \text { in the } 4 \text { - } \\
\text { month and } 759 \text { in the } 6 \text { - } \\
\text { month regimens ( } 88 \% \text { and } \\
87 \% \text { of those eligible, re- } \\
\text { spectively) } \\
\text { Culture using LJ solid media }\end{array}$ & $\begin{array}{l}\text { Reported for } 590 \\
(94 \%) \text { in the 4-month } \\
\text { and } 151 \\
(88 \%) \text { in the 6-month } \\
\text { regimens }\end{array}$ \\
\hline $\begin{array}{l}\text { Acquired } \\
\text { drug re- } \\
\text { sistance }\end{array}$ & Reported & Reported & Reported & Not reported & Not reported \\
\hline $\begin{array}{l}\text { Treat- } \\
\text { ment fail- } \\
\text { ure }\end{array}$ & $\begin{array}{l}\text { Includes culture confirmed and } \\
\text { not confirmed }\end{array}$ & $\begin{array}{l}\text { Includes culture con- } \\
\text { firmed and unconfirmed }\end{array}$ & Culture confirmed & $\begin{array}{l}\text { Includes culture confirmed } \\
\text { failure }\end{array}$ & Not reported \\
\hline
\end{tabular}




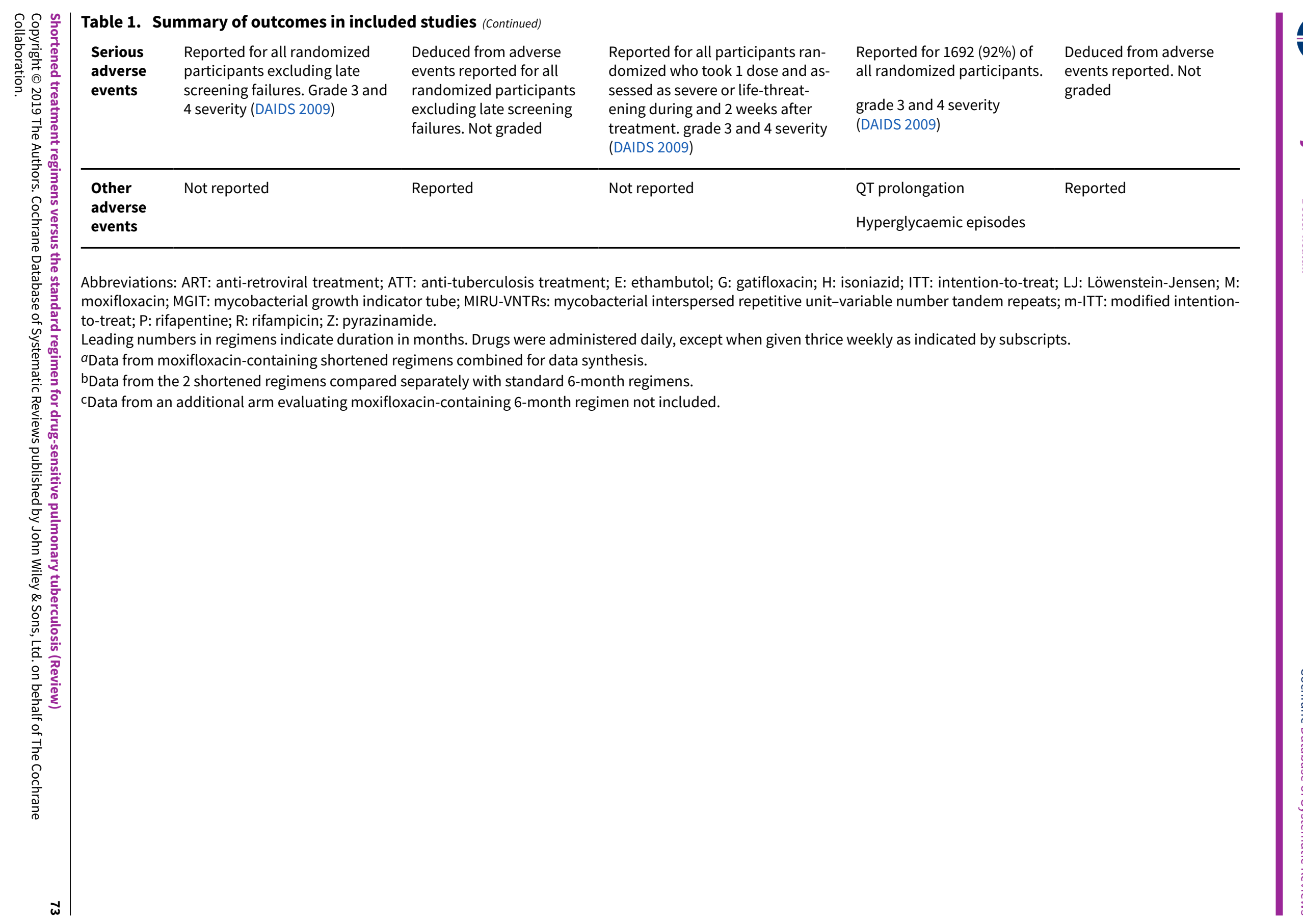


Table 2. Sensitivity analysis: moxifloxacin-based 4-month versus standard 6-month ATT regimens

\begin{tabular}{|c|c|c|c|c|c|c|}
\hline \multicolumn{7}{|l|}{ Primary outcome: relapse } \\
\hline \multirow{2}{*}{$\begin{array}{l}\text { Trial ID } \\
\text { Regimens }\end{array}$} & \multicolumn{2}{|l|}{ Gillespie 2014} & \multicolumn{2}{|c|}{ Jawahar 2013} & \multicolumn{2}{|c|}{ Jindani 2014} \\
\hline & 4 months & 6 months & 4 months & 6 months & 4 months & 6 months \\
\hline \multirow{2}{*}{$\begin{array}{l}a_{\text {Modified-ITT analysis (prima- }} \\
\text { ry analysis) }\end{array}$} & $110 / 1119$ & $13 / 555$ & $11 / 108$ & $10 / 155$ & $27 / 165$ & $6 / 163$ \\
\hline & $(9.8 \%)$ & $(2.3 \%)$ & $(10.1 \%)$ & $(6.5 \%)$ & $(16.4 \%)$ & $(3.7 \%)$ \\
\hline \multirow[t]{2}{*}{ aPer-protocol analysis } & $110 / 1038$ & $12 / 510$ & $11 / 107$ & $10 / 152$ & $26 / 165$ & $5 / 163$ \\
\hline & $(10.6 \%)$ & $(2.4 \%)$ & $(10.1 \%)$ & $(6.6 \%)$ & $(15.8 \%)$ & $(3.1 \%)$ \\
\hline \multirow{2}{*}{$\begin{array}{l}\text { bSensitivity analysis imputing } \\
\text { missing data }\end{array}$} & $126 / 1184$ & $14 / 577$ & $11 / 114$ & $10 / 159$ & $36 / 225$ & $71 / 232$ \\
\hline & $(10.7 \%)$ & $(2.4 \%)$ & $(9.7 \%)$ & $(6.3 \%)$ & $(16.0 \%)$ & $(2.6 \% \%)$ \\
\hline
\end{tabular}

Abbreviations: ATT: anti-tuberculosis treatment; ITT: intention-to-treat.

${ }^{a}$ As reported in trial reports.

bIncludes in the denominators for each trial arm all randomized participants minus those excluded post randomization due to ineligibility (not confirmed TB, or drug resistant), those who died, and those who experienced treatment failure. The difference in this denominator and the denominator in per-protocol analyses are missing data. Relapse rates for missing people were imputed from rates in the per-protocol analysis for each trial arm.

Table 3. Sensitivity analysis: gatifloxacin-based 4-month versus standard 6-month ATT regimens

\section{Primary outcome: relapse}

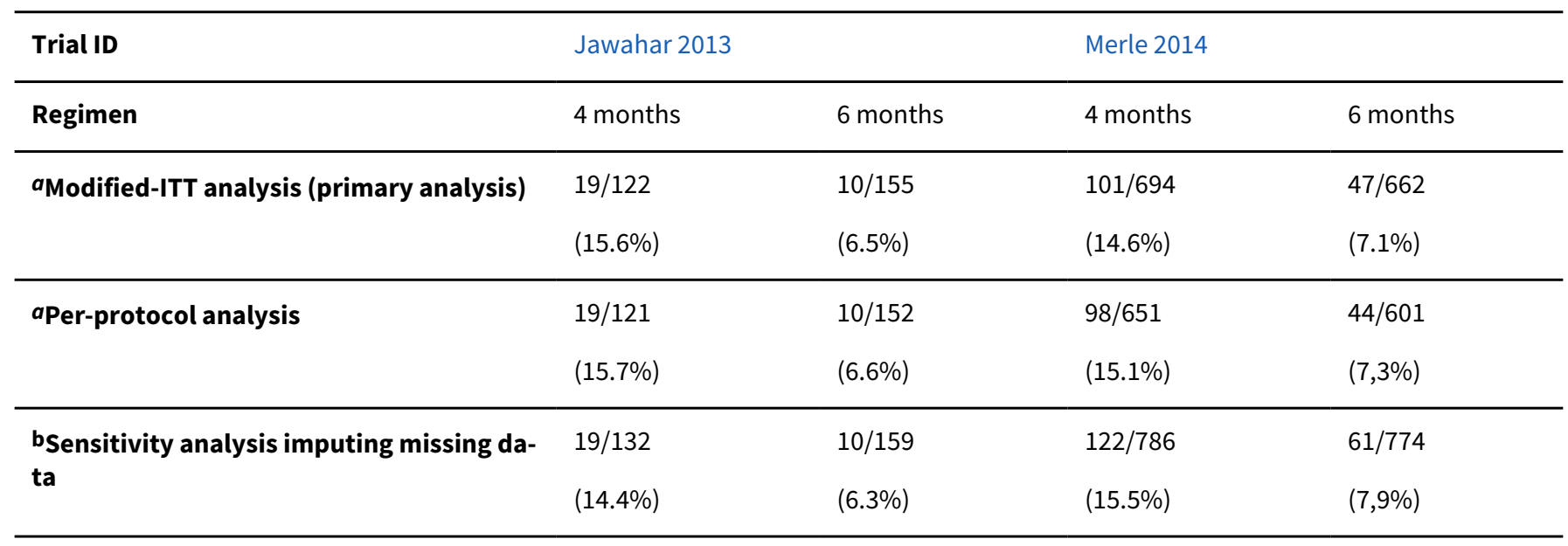

Abbreviations: ATT: anti-tuberculosis treatment; ITT: intention-to-treat.

$a_{\text {As reported in trial reports. }}$

bIncludes in the denominators for each trial arm all randomized participants minus those excluded post randomization due to ineligibility (not confirmed TB, or drug resistant), those who died, and those who experienced treatment failure. The difference in this denominator and the denominator in per-protocol analyses are missing data. Relapse rates for missing people were imputed from rates in the per-protocol analysis for each trial arm. 


\section{AP PEN DICES}

\section{Appendix 1. Search strategies}

\section{Cochrane Central Register of Controlled Trials}

\section{Issue 10, July 2019}

ID Search

\#1 (tuberculosis or TB) and (pulmonary or lung):ti,ab,kw

\#2 MeSH descriptor: [Tuberculosis, Pulmonary] explode all trees

\#3 \#1 or \#2

\#4 moxifloxacin or levofloxacin or gatifloxacin or rifamycins or rifabutin or rifapentine or bedaquiline or delamanid or pretomanid:ti,ab,kw \#5 diarylquinolin* or TMC 207-BDQ or nitroimidazol* or PA 824- pretomanid or oxazolidinon* or LZD or ethylenediamin* or SQ 109 \#6 MeSH descriptor: [Antitubercular Agents] explode all trees

\#7 MeSH descriptor: [Diarylquinolines] explode all trees

\#8 MeSH descriptor: [Fluoroquinolones] explode all trees

\#9 \#4 or \#5 or \#6 or \#7 or \#8

$\# 10 \# 3$ and \#9

\#11 regimen* or short or shortened or months or dose or dosing or schedule*

\#12 regimen* or short or shortened or months or dose or dosing or schedule*:ti,ab,kw (Word variations have been searched)

\#13 MeSH descriptor: [Drug Administration Schedule] explode all trees

\#14 MeSH descriptor: [Medication Therapy Management] explode all trees

\#15 MeSH descriptor: [Time Factors] explode all trees

$\# 16 \# 12$ or \#13 or \#14 or \#15

$\# 17$ \#10 and \#16

PubMed (MEDLINE)

\begin{tabular}{ll}
\hline Search & Query \\
\hline$\# 1$ & Search "Tuberculosis, Pulmonary"[Mesh] \\
\hline$\# 2$ & Search pulmonary and (tuberculosis or TB) Field: Title/Abstract \\
\hline$\# 3$ & Search (\#2) OR \#1 \\
\hline$\# 4$ & $\begin{array}{l}\text { Search "Antitubercular Agents"[Mesh] OR "Diarylquinolines"[Mesh] OR "Antibiotics, Antitubercu- } \\
\text { lar"[Mesh] }\end{array}$ \\
\hline$\# 5$ & $\begin{array}{l}\text { Search diarylquinolin* or TMC 207-BDQ or nitroimidazol* or PA 824- pretomanid or oxazolidinon* } \\
\text { or LZD or ethylenediamin* or SQ 109 Field: Title/Abstract }\end{array}$ \\
\hline$\# 6$ & $\begin{array}{l}\text { Search moxifloxacin or levofloxacin or gatifloxacin or rifamycins or rifabutin or rifapentine or be- } \\
\text { daquiline or delamanid or pretomanid Field: Title/Abstract }\end{array}$ \\
\hline
\end{tabular}


(Continued)

\begin{tabular}{|c|c|}
\hline \#7 & Search ((\#6) OR \#5) OR \#4 \\
\hline \#8 & Search "Drug Administration Schedule"[Mesh] \\
\hline \#9 & Search "Medication Therapy Management"[Mesh] \\
\hline \#10 & Search "Time Factors"[Mesh] \\
\hline$\# 11$ & $\begin{array}{l}\text { Search regimen* or short or shortened or months or dose or dosing or schedule* Field: Title/Ab- } \\
\text { stract }\end{array}$ \\
\hline \#12 & Search $(((\# 11)$ OR \#10) OR \#9) OR \#8 \\
\hline \#13 & Search (\#12) AND \#7 AND \#3 \\
\hline$\# 15$ & Search randomized or placebo or randomly or trial or groups Field: Title/Abstract \\
\hline \#16 & Search drug therapy [sh] \\
\hline \#17 & Search ((\#14) OR \#15) OR \#16 \\
\hline \#18 & Search (\#17) AND \#13 \\
\hline
\end{tabular}

\section{Embase 1947-Present, updated daily}

Search Strategy:

1 pulmonary tuberculosis.mp. or lung tuberculosis/

2 (moxifloxacin or levofloxacin or gatifloxacin or rifamycins or rifabutin or rifapentine or bedaquiline or delamanid or pretomanid).ab. or (moxifloxacin or levofloxacin or gatifloxacin or rifamycins or rifabutin or rifapentine or bedaquiline or delamanid or pretomanid).ti.

3 (diarylquinolin* or TMC 207-BDQ or nitroimidazol* or PA 824- pretomanid or oxazolidinon* or LZD or ethylenediamin* or SQ 109).ab. or (diarylquinolin* or TMC 207-BDQ or nitroimidazol* or PA 824- pretomanid or oxazolidinon* or LZD or ethylenediamin* or SQ 109).ti.

4 antitubercular agents.mp. or *tuberculostatic agent/

5 *quinoline derivative/

6 fluoroquinolones.mp. or * quinolone derivative/

72 or 3 or 4 or 5 or 6

81 and 7

9 (regimen* or short or shortened or months or dose or dosing or schedule*).ab. or (regimen* or short or shortened or months or dose or dosing or schedule*).ti.

108 and 9

11 randomized controlled trial/

12 controlled clinical trial/

13 (randomized or controlled or placebo or double-blind* or single-blind*).mp. 
1411 or 12 or 13

1510 and 14

16 limit 15 to human

\section{LILACS}

(tw:(pulmonary tuberculosis)) AND (tw:(diarylquinolin* OR tmc 207-bdq OR nitroimidazol ${ }^{\star}$ OR pa 824- pretomanid OR oxazolidinon* OR Izd OR ethylenediamin* OR sq 109 OR moxifloxacin OR levofloxacin OR gatifloxacin OR rifamycins OR rifabutin OR rifapentine OR bedaquiline OR delamanid OR pretomanidor )) AND (tw:(regimen* OR short OR shortened OR months OR dose OR dosing OR schedule* )) AND (instance:"regional")

\section{Web of Science}

\section{Set}

\begin{tabular}{ll}
\hline$\# 6$ & $\# 5$ AND \#4 AND \#3 \\
& Indexes=SCI-EXPANDED, CPCI-S Timespan=All years \\
\hline
\end{tabular}

\# 5 TOPIC: (randomized or controlled or trial or double-blind or single-blind)

Indexes=SCI-EXPANDED, CPCI-S Timespan=All years

\#4 TOPIC: (regimen* or short or shortened or months or dose or dosing or schedule*)

Indexes=SCI-EXPANDED, CPCI-S Timespan=All years

$\begin{array}{ll}\# 3 & \# 2 \text { AND \#1 } \\ \text { Indexes=SCI-EXPANDED, CPCI-S Timespan=All years }\end{array}$

\# 2 TOPIC: (moxifloxacin or levofloxacin or gatifloxacin or rifamycins or rifabutin or rifapentine or bedaquiline or delamanid or pretomanid) OR TOPIC: (diarylquinolin* or TMC 207-BDQ or nitroimidazol* $^{\star}$ or PA 824- pretomanid or oxazolidinon* or LZD or ethylenediamin* or SQ 109) OR TOPIC: (antitubercular)

Indexes=SCI-EXPANDED, CPCI-S Timespan=All years

TOPIC: (pulmonary tuberculosis)

Indexes=SCI-EXPANDED, CPCI-S Timespan=All years

\section{IndMed}

"pulmonary tuberculosis" AND regime ${ }^{\star}$ or schedule*

\section{South Asian Database of Controlled trials (SADCCT)}

"pulmonary tuberculosis" AND regime* or schedule*

\section{ClinicalTrials.gov}

"pulmonary tuberculosis" and regime*, "pulmonary tuberculosis" and shortened, "pulmonary tuberculosis" and schedule*

\section{WHO ICTRP}

pulmonary tuberculosis" and regime*, "pulmonary tuberculosis" and shortened , "pulmonary tuberculosis" and schedule* 


\section{CONTRIBUTIONS OF AUTHORS}

AG assessed eligibility, extracted data, assessed risk of bias, undertook meta-analysis, assessed certainty of evidence using the GRADE approach, and drafted the review. AM assessed eligibility, extracted data, helped assess risk of bias, and helped write the review. SJ, JT, and SS helped write the review. RK helped extract data and helped with data synthesis and assessing certainty of the evidence. PT checked eligibility and data extraction, helped with data synthesis, helped assess the certainty of evidence, and helped write the review. All review authors approved the final version of this review.

\section{DECLARATIONS OF INTEREST}

AG has no known conflicts of interest.

AM has no known conflicts of interest.

SJ has no known conflicts of interest.

JT has no known conflicts of interest.

SS has no known conflicts of interest.

PT supported by the grant previous to READ-It (Grant: 5242). He was contracted by Cochrane to help build capacity among authors from India and the region to undertake systematic reviews.

RK supported by the grant previous to READ-It (Grant: 5242), and has no known conflicts of interest.

\section{SOURCES OF SUPPORT}

\section{Internal sources}

- Sree Balaji Medical College and Hospital, India.

Employment for Angeline G Grace

- All India Institute of Medical Sciences, New Delhi, India.

Employment for Abhenil Mittal

- Postgraduate Institute of Medical Education and Research, Chandigarh, India.

Employment for Siddharth Jain

- International Union Against Tuberculosis and Lung Disease (The Union), South-East Asia Regional Office, India.

Employment for Jaya P Tripathy and Srinath Sathyanarayana

- Christian Medical College, Vellore, India.

Employment for Prathap Tharyan and logistic support for the Prof BV Moses Center for Evidence-Informed Health Care and Health Policy that hosted Cochrane South Asia during developing the protocol and initiating the review. Salary support for Richard Kirubakaran via the Prof. BV Moses Centre

- Liverpool School of Tropical Medicine, UK.

\section{External sources}

- Department for International Development (DFID), UK.

Project number 300342-104

\section{DIFFERENCES BETWEEN PROTOCOL AND REVIEW}

In the methods, under 'Unit of analysis issues', in the protocol, we had stated that, "When a multi-arm study contributes multiple comparisons to a particular meta-analysis, for dichotomous data we will split the 'shared' group data appropriately to avoid double counting". In the review, this was considered inappropriate. Instead, from trials with multiple intervention arms, we undertook pair-wise comparisons of only relevant interventions and when possible combined the results of trial arms with similar ATT regimens.

In the protocol, we had stated that we will carry out a complete case analysis and will explore the effects of missing data in a series of 'best-worst case' sensitivity analyses. For the review, we believed that these assumptions would be too extreme when outcomes were rare. Instead we assessed the impact of missing data as described in the sections Dealing with missing data and Sensitivity analysis.

In the protocol, we had stated that we would "use the $\mathrm{I}^{2}$ statistic with a value of $50 \%$ or greater as denoting significant heterogeneity in the results (intertrial variability that exceeds random error). However, if an opposite direction of effect estimates and gross non-overlapping of confidence intervals of individual trials are observed, we may lower the acceptable level of heterogeneity to an $1^{2}$ statistic of $30 \% "$. In the review, we clarified this in accordance with current guidance in the Cochrane Handbook for Systematic Reviews of Interventions, regarding interpreting the $\mathrm{I}^{2}$ statistic (Deeks 2011). We had also stated that we would conduct meta-analyses using a fixed-effect model 
when heterogeneity was low and a random-effects model when heterogeneity was moderate. However, in the review, in keeping with current guidance regarding the $\mathrm{I}^{2}$ statistic, if inconsistency was judged to be moderate but due to large and small effects favouring an intervention, and with overlapping 95\% confidence intervals, we used a random-effects model in sensitivity analyses. If this did not change the direction of effect, nor result in imprecision in effect estimates (because the wider $95 \% \mathrm{Cls}$ with the random-effects model included non-appreciable and appreciable benefits), we retained the fixed-effect model in meta-analysis but additionally reported the randomeffects meta-analysis in the results.

In the protocol, we had stated that if we identify significant heterogeneity, one of the potential sources we would explore in subgroup analysis for the primary outcome measure was the category of the shortened treatment regimen (fluoroquinolone-based and nonfluoroquinolone-based). We did not find non-fluoroquinolone-based shorter ATT regimens. We therefore explored potential causes of heterogeneity in subgroup analyses based on categories of shortened treatment regimens. We could not undertake subgroup analysis of trials that included adults and those that included children because the included trials recruited only adults.

For the review, we explained in greater detail the sensitivity analyses we would undertake to explore moderate heterogeneity.

For the review, we expanded the section describing the methods of assessing 'Certainty of evidence' to improve transparency and clarity.

I N D EX TERMS

\section{Medical Subject Headings (MeSH)}

Antitubercular Agents [ ${ }^{\star}$ therapeutic use]; Clinical Protocols; Drug Administration Schedule; Drug Combinations; Drug Therapy, Combination [methods]; Randomized Controlled Trials as Topic; Tuberculosis, Pulmonary [ ${ }^{\star}$ drug therapy]

\section{MeSH check words}

Humans 